\title{
Height pairings on orthogonal Shimura varieties
}

\author{
Fabrizio Andreatta, Eyal Z. Goren, Benjamin Howard and Keerthi \\ Madapusi Pera
}

\begin{abstract}
Let $M$ be the Shimura variety associated to the group of spinor similitudes of a quadratic space over $\mathbb{Q}$ of signature $(n, 2)$. We prove a conjecture of Bruinier and Yang, relating the arithmetic intersection multiplicities of special divisors and CM points on $M$ to the central derivatives of certain $L$-functions. Each such $L$-function is the RankinSelberg convolution associated with a cusp form of half-integral weight $n / 2+1$, and the weight $n / 2$ theta series of a positive definite quadratic space of rank $n$.

When $n=1$ the Shimura variety $M$ is a classical quaternionic Shimura curve, and our result is a variant of the Gross-Zagier theorem on heights of Heegner points.
\end{abstract}

\section{Contents}

1 Introduction 1

2 The GSpin Shimura variety and its special divisors 6

3 Harmonic modular forms and special divisors 25

4 Complex multiplication cycles $\quad 29$

5 Degrees of metrized line bundles $\quad 41$

$\begin{array}{ll}\text { References } & 57\end{array}$

\section{Introduction}

In this paper we prove a generalization, conjectured by Bruinier-Yang [BY09], of the Gross-Zagier theorem on heights of Heegner points on modular curves.

Instead of a modular curve we work on the Shimura variety defined by a reductive group over $\mathbb{Q}$ of type $\operatorname{GSpin}(n, 2)$. The Heegner points are replaced by 0 -cycles arising from embeddings of rank two tori into $\operatorname{GSpin}(n, 2)$, and by divisors arising from embeddings of $\operatorname{GSpin}(n-1,2)$ into $\operatorname{GSpin}(n, 2)$. We prove that the arithmetic intersections of these special cycles are related to the central derivatives of certain Rankin-Selberg convolution $L$-functions.

\subsection{The Shimura variety}

Fix an integer $n \geqslant 1$ and a quadratic space $(V, Q)$ over $\mathbb{Q}$ of signature $(n, 2)$. From $V$ one can construct a Shimura datum $(G, \mathcal{D})$, in which $G=\operatorname{GSpin}(V)$ is a reductive group over $\mathbb{Q}$ sitting in an exact sequence

$$
1 \rightarrow \mathbb{G}_{m} \rightarrow \operatorname{GSpin}(V) \rightarrow \mathrm{SO}(V) \rightarrow 1
$$

F. Andreatta is supported by the Italian grant Prin 2010/2011. E. Z. Goren is supported by NSERC discovery grant, B. Howard is supported by NSF grant DMS-1201480. K. Madapusi Pera is supported by NSF Postdoctoral Research Fellowship DMS-1204165. 


\section{F. Andreatta, E. Z. Goren, B. Howard, K. Madapusi Pera}

and the hermitian domain $\mathcal{D}$ is an open subset of the space of isotropic lines in $\mathbb{P}^{1}\left(V_{\mathbb{C}}\right)$.

Any choice of lattice $L \subset V$ on which $Q$ is $\mathbb{Z}$-valued determines a compact open subgroup $K \subset G\left(\mathbb{A}_{f}\right)$. We fix such a lattice, and assume that $L$ is maximal, in the sense that it admits no superlattice on which $Q$ is $\mathbb{Z}$-valued. The data $L \subset V$ now determines a complex orbifold

$$
M(\mathbb{C})=G(\mathbb{Q}) \backslash \mathcal{D} \times G\left(\mathbb{A}_{f}\right) / K
$$

which, by the theory of canonical models of Shimura varieties, is the space of complex points of an algebraic stack $M \rightarrow \operatorname{Spec}(\mathbb{Q})$. (Throughout this article stack means Deligne-Mumford stack.)

Except for small values of $n$, the Shimura variety $M$ is not of PEL type; that is, it is not naturally a moduli space of abelian varieties with polarization, endomorphisms, and level structures. It is, however, of Hodge type, and so the recent work of Kisin [Kis10] (and its extension in [MP16] and [KM15]) provides us with a regular and flat integral model

$$
\mathcal{M} \rightarrow \operatorname{Spec}(\mathbb{Z})
$$

\subsection{Special divisors}

The integral model carries over it a canonical family of abelian varieties: the Kuga-Satake abelian scheme

$$
\mathcal{A} \rightarrow \mathcal{M}
$$

The Kuga-Satake abelian scheme is endowed with an action of the Clifford algebra $C(L)$, and with a $\mathbb{Z} / 2 \mathbb{Z}$-grading $\mathcal{A}=\mathcal{A}^{+} \times \mathcal{A}^{-}$.

For any scheme $S \rightarrow \mathcal{M}$, the pullback $\mathcal{A}_{S}$ comes with a distinguished $\mathbb{Z}$-module

$$
V\left(\mathcal{A}_{S}\right) \subset \operatorname{End}\left(\mathcal{A}_{S}\right)
$$

of special endomorphisms, and there is a positive definite quadratic form

$$
Q: V\left(\mathcal{A}_{S}\right) \rightarrow \mathbb{Z}
$$

characterized by $x \circ x=Q(x) \cdot \mathrm{Id}$. Slightly more generally, there is a distinguished subset

$$
V_{\mu}\left(\mathcal{A}_{S}\right) \subset V\left(\mathcal{A}_{S}\right) \otimes_{\mathbb{Z}} \mathbb{Q}
$$

for each coset $\mu \in L^{\vee} / L$, where $L^{\vee}$ is the dual lattice of $L$. Taking $\mu=0$ recovers $V\left(\mathcal{A}_{S}\right)$.

The special endomorphisms allow us to define a family of special divisors on $\mathcal{M}$. For $m \in \mathbb{Q}>0$ and $\mu \in L^{\vee} / L$, let $\mathcal{Z}(m, \mu) \rightarrow \mathcal{M}$ be the moduli stack that assigns to any $\mathcal{M}$-scheme $S \rightarrow \mathcal{M}$ the set

$$
\mathcal{Z}(m, \mu)(S)=\left\{x \in V_{\mu}\left(\mathcal{A}_{S}\right): Q(x)=m\right\} .
$$

The morphism $\mathcal{Z}(m, \mu) \rightarrow \mathcal{M}$ is relatively representable, finite, and unramified, and allows us to view $\mathcal{Z}(m, \mu)$ as a Cartier divisor on $\mathcal{M}$.

In the generic fiber of $\mathcal{M}$ the special divisors agree with the divisors appearing in [Bor98], [Bru02], [BY09], and [Kud04]. In special cases where $V$ has signature $(1,2),(2,2)$, or $(3,2)$, the Shimura variety $\mathcal{M}$ is (a quaternionic version of) a modular curve, Hilbert modular surface, or Siegel threefold, respectively. In these cases the special divisors are traditionally known as complex multiplication points, Hirzebruch-Zagier divisors, and Humbert surfaces. See, for example, [vdG88], [KR99], [KR00], and [KRY06]. 


\subsection{CM points}

Let $V_{0} \subset V$ be a negative definite plane in $V$, and set $L_{0}=V_{0} \cap L$. The Clifford algebra $C\left(L_{0}\right)$ is an order in a quaternion algebra over $\mathbb{Q}$, and its even part $C^{+}\left(L_{0}\right) \subset C\left(L_{0}\right)$ is an order in a quadratic imaginary field $\boldsymbol{k}$. Fix an embedding $\boldsymbol{k} \rightarrow \mathbb{C}$, and assume from now on that $C^{+}\left(L_{0}\right)=\mathcal{O}_{\boldsymbol{k}}$ is the maximal order in $\boldsymbol{k}$.

The group $\operatorname{GSpin}\left(V_{0}\right) \cong \operatorname{Res}_{\boldsymbol{k} / \mathbb{Q}} \mathbb{G}_{m}$ is a rank two torus, and has associated to it a 0 dimensional Shimura variety $Y \rightarrow \operatorname{Spec}(\boldsymbol{k})$, which can be reinterpreted as the moduli stack of elliptic curves with $\mathrm{CM}$ by $\mathcal{O}_{\boldsymbol{k}}$. This allows us to construct a smooth integral model $\mathcal{Y} \rightarrow \operatorname{Spec}\left(\mathcal{O}_{\boldsymbol{k}}\right)$. If $\boldsymbol{k}$ has odd discriminant the inclusion $V_{0} \rightarrow V$ induces an embedding $\operatorname{GSpin}\left(V_{0}\right) \rightarrow G$ and a relatively representable, finite, and unramified morphism

$$
i: \mathcal{Y} \rightarrow \mathcal{M}_{\mathcal{O}_{k}} .
$$

The stack $\mathcal{Y}$ has its own Kuga-Satake abelian scheme $\mathcal{A}_{0} \rightarrow \mathcal{Y}$, endowed with a $\mathbb{Z} / 2 \mathbb{Z}$-grading $\mathcal{A}_{0} \cong \mathcal{A}_{0}^{+} \times \mathcal{A}_{0}^{-}$, and an action of $C\left(L_{0}\right)$. Here $\mathcal{A}_{0}^{+}$is the universal elliptic curve with CM by $\mathcal{O}_{k}$ and $\mathcal{A}_{0} \cong \mathcal{A}_{0}^{+} \otimes_{\mathcal{O}_{k}} C\left(L_{0}\right)$. It is related to the Kuga-Satake abelian scheme $\mathcal{A} \rightarrow \mathcal{M}$ by a $C(L)$-linear isomorphism

$$
\left.\left.\mathcal{A}\right|_{\mathcal{Y}} \cong \mathcal{A}_{0}\right|_{\mathcal{Y}} \otimes_{C\left(L_{0}\right)} C(L)
$$

Let $\widehat{\operatorname{Pic}}(\mathcal{M})$ denote the group of metrized line bundles on $\mathcal{M}$, and similarly with $\mathcal{M}$ replaced by $\mathcal{M}_{\mathcal{O}_{k}}$ or $\mathcal{Y}$. The composition

$$
\widehat{\operatorname{Pic}}(\mathcal{M}) \rightarrow \widehat{\operatorname{Pic}}\left(\mathcal{M}_{\mathcal{O}_{k}}\right) \stackrel{i^{*}}{\rightarrow} \widehat{\operatorname{Pic}}(\mathcal{Y}) \stackrel{\widehat{\operatorname{deg}}}{\rightarrow} \mathbb{R}
$$

is denoted $\widehat{\mathcal{Z}} \mapsto[\widehat{\mathcal{Z}}: \mathcal{Y}]$. We call it arithmetic degree along $\mathcal{Y}$.

\subsection{Harmonic modular forms}

Denote by $\widetilde{\mathrm{SL}_{2}}(\mathbb{Z})$ the metaplectic double cover of $\mathrm{SL}_{2}(\mathbb{Z})$, and by

$$
\omega_{L}: \widetilde{\mathrm{SL}}_{2}(\mathbb{Z}) \rightarrow \operatorname{Aut}_{\mathbb{C}}\left(\mathfrak{S}_{L}\right)
$$

the Weil representation on the space $\mathfrak{S}_{L}$ of $\mathbb{C}$-valued functions on $L^{\vee} / L$. There is a complex conjugate representation $\bar{\omega}_{L}$ on the same space, and a contragredient action $\omega_{L}^{\vee}$ on the dual space $\mathfrak{S}_{L}^{\vee}$.

Bruinier and Funke [BF04] have defined a surjective conjugate-linear differential operator

$$
\xi: H_{1-\frac{n}{2}}\left(\omega_{L}\right) \rightarrow S_{1+\frac{n}{2}}\left(\bar{\omega}_{L}\right) .
$$

Here $S_{1+\frac{n}{2}}\left(\bar{\omega}_{L}\right)$ is the space of weight $1+\frac{n}{2}$ cusp forms valued in $\mathfrak{S}_{L}$, and transforming according to the representation $\bar{\omega}_{L}$. The space $H_{1-\frac{n}{2}}\left(\omega_{L}\right)$ is defined similarly, but the forms are harmonic weak Mass forms in the sense of [BY09, $\S 3.1]$.

To any $f \in H_{1-\frac{n}{2}}\left(\omega_{L}\right)$ there is associated a formal $q$-expansion

$$
f^{+}(\tau)=\sum_{\substack{m \in \mathbb{Q} \\ m \gg-\infty}} c_{f}^{+}(m) q^{m} \in \mathfrak{S}_{L}[[q]],
$$

and each coefficient is uniquely a linear combination $\sum_{\mu} c_{f}^{+}(m, \mu) \varphi_{\mu}$ of the characteristic functions $\varphi_{\mu}$ of cosets $\mu \in L^{\vee} / L$. Assuming that $f^{+}$has integral principal part, in the sense that 
$c_{f}^{+}(m, \mu) \in \mathbb{Z}$ whenever $m<0$, we may form the Cartier divisor

$$
\mathcal{Z}(f)=\sum_{m>0} \sum_{\mu \in L^{\vee} / L} c_{f}^{+}(-m, \mu) \cdot \mathcal{Z}(m, \mu)
$$

(the sum is finite) on the integral model $\mathcal{M}$, following the work of Borcherds [Bor98], Bruinier [Bru02], and Bruinier-Yang [BY09].

Using the formalism of regularized theta lifts developed in [Bor98], Bruinier [Bru02] defined a Green function $\Phi(f)$ for $\mathcal{Z}(f)$. This Green function determines a metric on the corresponding line bundle, yielding a metrized line bundle

$$
\widehat{\mathcal{Z}}(f) \in \widehat{\operatorname{Pic}}(\mathcal{M}) \text {. }
$$

\subsection{The main result}

The central problem is to compute the arithmetic degree $[\widehat{\mathcal{Z}}(f): \mathcal{Y}]$. Assuming that the divisor $\mathcal{Z}(f)_{\mathcal{O}_{k}}$ intersects the cycle $\mathcal{Y}$ properly, the arithmetic degree decomposes as a sum of local contributions. The calculation of the archimedean contribution, which is essentially the sum of the values of $\Phi(f)$ at all $y \in \mathcal{Y}(\mathbb{C})$, is the main result of [BY09].

Based on their calculation of the archimedean contribution, Bruinier and Yang formulated a conjecture [BY09, Conjecture 1.1] relating $[\widehat{\mathcal{Z}}(f): \mathcal{Y}]$ to the derivative of an $L$-function. Our main result is a proof of this conjecture.

The statement of the result requires two more ingredients. The first is the metrized cotautological bundle

$$
\widehat{T} \in \widehat{\operatorname{Pic}}(\mathcal{M}) .
$$

By definition, the hermitian domain $\mathcal{D}$ is an open subset of the space of isotropic lines in $\mathbb{P}^{1}\left(V_{\mathbb{C}}\right)$. Restricting the tautological bundle on $\mathbb{P}^{1}\left(V_{\mathbb{C}}\right)$ yields a line bundle $\omega_{\mathcal{D}}$ on $\mathcal{D}$, which descends first to the orbifold $M(\mathbb{C})$, and then to the canonical model $M$. The resulting line bundle, $\omega$, is the tautological bundle on $M$, also called the line bundle of weight one modular forms. There is a natural metric (5.8) on $\omega$, and our $\widehat{\boldsymbol{T}}$ is an integral model of $\widehat{\omega}^{-1}$.

The second ingredient is a classical vector valued theta series. From the $\mathbb{Z}$-quadratic space

$$
\Lambda=\left\{x \in L: x \perp L_{0}\right\}
$$

of signature $(n, 0)$, one can construct a half-integral weight theta series

$$
\Theta_{\Lambda}(\tau) \in M_{\frac{n}{2}}\left(\omega_{\Lambda}^{\vee}\right)
$$

Here $\omega_{\Lambda}$ is the Weil representation of $\widetilde{\mathrm{SL}}_{2}(\mathbb{Z})$ on the space $\mathfrak{S}_{\Lambda}$ of functions on $\Lambda^{\vee} / \Lambda$, and $\omega_{\Lambda}^{\vee}$ is its contragredient. See $\S 3.3$ for the precise definition.

The following appears in the text as Theorem 5.7.3.

Theorem A. Assume that the discriminant of $\boldsymbol{k}$ is odd, and let $h_{\boldsymbol{k}}$ and $w_{\boldsymbol{k}}$ be the class number and number of roots of unity in $\boldsymbol{k}$, respectively. Every weak harmonic Maass form $f \in H_{1-n / 2}\left(\omega_{L}\right)$ with integral principal part satisfies

$$
[\widehat{\mathcal{Z}}(f): \mathcal{Y}]+c_{f}^{+}(0,0) \cdot[\widehat{\boldsymbol{T}}: \mathcal{Y}]=-\frac{h_{\boldsymbol{k}}}{w_{\boldsymbol{k}}} \cdot L^{\prime}\left(\xi(f), \Theta_{\Lambda}, 0\right),
$$

where $c_{f}^{+}(0,0)$ is the value of $c_{f}^{+}(0) \in \mathfrak{S}_{L}$ at the trivial coset in $L^{\vee} / L$, and $L\left(\xi(f), \Theta_{\Lambda}, s\right)$ is the Rankin-Selberg convolution $L$-function of $\S 3.3$. 


\section{HEIGHT PAIRINGS ON ORTHOGONAL SHIMURA VARIETIES}

Remark 1.5.1. The analogous theorem for Shimura varieties associated with unitary similitude groups was proved in [BHY15].

Remark 1.5.2. The convolution $L$-function appearing in the theorem can be realized by integrating $\xi(f)$ and $\Theta_{\Lambda}$ against a certain Eisenstein series $E_{L_{0}}(\tau, s)$. This Eisenstein series vanishes at $s=0$, and hence the same is true of $L\left(\xi(f), \Theta_{\Lambda}, s\right)$.

Remark 1.5.3. As noted earlier, our cycles $\mathcal{Y} \rightarrow \mathcal{M}_{\mathcal{O}_{k}}$ arise from embeddings of rank two tori into $\operatorname{GSpin}(n, 2)$. One could instead look at the cycles defined by embeddings of maximal tori; these are the big CM cycles of [BKY12]. In [AGHM15] we prove a result analogous to Theorem A in this setting. We show that it implies an averaged form of Colmez's conjecture on the Faltings heights of CM abelian varieties (via the method of Yang [Yan10]). This has provided a key ingredient in J. Tsimerman's recent proof of the André-Oort conjecture [Tsi15], which characterizes Shimura subvarieties of Shimura varieties of Hodge type by means of special points.

Remark 1.5.4. If one is willing to have the equality of Theorem A only up to rational multiples of $\log (2)$, in principle it should be possible to drop the assumption that $d_{\boldsymbol{k}}$ is odd or that $C^{+}\left(L_{0}\right)$ is maximal and replace it by the weaker assumption that $C^{+}\left(L_{0}\right)$ is maximal away from 2 . Such improvements come at a cost: under these weaker hypotheses one does not know anything like the explicit formulas appearing in Proposition 3.3.1 and Theorem 4.5.1.

Instead, one should be able (we have not checked the details) to prove the main result by using the Siegel-Weil arguments of [BKY12] and [KY13]. In essence, the Siegel-Weil formula allows one to compare the point counting part of Theorem 4.5.1 with the values of certain Whittaker functions appearing in the Eisenstein series coefficients of Proposition 3.3.1, without deriving explicit formulas for either one.

A rough outline of the contents is as follows:

In $\S 2$ we establish the basic properties of the Shimura variety $\mathcal{M}$ on which we will be performing intersection theory, and of the special cycles $\mathcal{Z}(m, \mu)$. We prove that the $\mathcal{Z}(m, \mu)$ define Cartier divisors on $\mathcal{M}$, and explore the functoriality of the formation of $\mathcal{M}$ and its divisors with respect to the quadratic space $L$. More precisely, given an isometric embedding $L_{0} \hookrightarrow L$ of maximal $\mathbb{Z}$-quadratic spaces of signatures $\left(n_{0}, 2\right)$ and $(n, 2)$, with $1 \leqslant n_{0} \leqslant n$, we prove the existence of a canonical morphism $\mathcal{M}_{0} \rightarrow \mathcal{M}$ between the associated integral models, and show that the pullback of a special divisor on $\mathcal{M}$ is a prescribed linear combination of special divisors on $\mathcal{M}_{0}$.

In $\S 3$ we remind the reader of some of the analytic theory used in [BY09]. In particular, we recall the essential properties of harmonic weak Maass forms, the divisors $Z(f)$ on $M$ associated to such forms, and the Green functions $\Phi(f)$ for these divisors, which are defined as regularized theta lifts. We also recall the theta series and convolution $L$-functions that appear in the main theorem, and Schofer's [Sch09] calculation of the Fourier coefficients of the Eisenstein series of Remark 1.5.2. The only thing new here is that, thanks to the constructions of $\S 2$, we are able to define an extension of the divisor $Z(f)$ on $M$ to a divisor $\mathcal{Z}(f)$ on the integral model $\mathcal{M}$.

In $\S 4$ we fix a quadratic space $L_{0}$ over $\mathbb{Z}$ of signature $(0,2)$, whose even Clifford algebra is isomorphic to the maximal order in a quadratic imaginary field $\boldsymbol{k}$. The Clifford algebra of $L_{0}$ then has the form $C\left(L_{0}\right) \stackrel{\simeq}{\rightarrow} \mathcal{O}_{\boldsymbol{k}} \oplus L_{0}$. The Shimura variety associated to the rank two torus $\operatorname{GSpin}\left(L_{0 \mathbb{Q}}\right)$ has dimension 0 , and can be realized as the moduli stack of elliptic curves with complex multiplication by $\mathcal{O}_{k}$. Using this interpretation we define an integral model $\mathcal{Y} \rightarrow$ $\operatorname{Spec}\left(\mathcal{O}_{\boldsymbol{k}}\right)$, and define the Kuga-Satake abelian scheme as $\mathcal{A}_{0}=\mathcal{E} \otimes_{\mathcal{O}_{\boldsymbol{k}}} C\left(L_{0}\right)$, where $\mathcal{E} \rightarrow \mathcal{Y}$ is the universal CM elliptic curve. All of the results of $\S 2$ are then extended to this new setting. 
In particular, we define a family of divisors $\mathcal{Z}_{0}(m, \mu)$ on the arithmetic curve $\mathcal{Y}$, each of which can be characterized as the locus of points where $\mathcal{A}_{0}$ has an extra quasi-endomorphism with prescribed properties. This can also be expressed in terms of endomorphisms of the universal CM elliptic curve: along each special divisor the action of $\mathcal{O}_{\boldsymbol{k}}$ on $\mathcal{E}$ extends to an action of an order in a definite quaternion algebra. Theorem 4.5.1 gives explicit formulas for the degrees of the divisors $\mathcal{Z}_{0}(m, \mu)$, and these degrees match up with Schofer's formulas for the coefficients of Eisenstein series. Many cases of Theorem 4.5.1 already appear in work of Kudla-RapoportYang [KRY99] and Kudla-Yang [KY13], and our proof, like theirs, makes essential use of Gross's calculation [Gro86] of the endomorphism rings of canonical lifts of CM elliptic curves.

In $\S 5$ we prove Theorem A. The greatest difficulty comes from the fact that the 1-cycle $\mathcal{Y}$ on $\mathcal{M}_{\mathcal{O}_{k}}$ may have irreducible components contained entirely within $\mathcal{Z}(m, \mu)_{\mathcal{O}_{k}}$. Thus we must compute improper intersections. Our techniques for doing this are a blend of the methods used in [BHY15] on unitary Shimura varieties, and the methods of Hu's thesis [Hu99], which reconstructs the arithmetic intersection theory of Gillet-Soulé [GS90] using Fulton's method of deformation to the normal cone.

The cycle $\mathcal{Y}$ has a normal bundle $N_{\mathcal{Y}} \mathcal{M} \rightarrow \mathcal{Y}$, and the arithmetic divisor $\widehat{\mathcal{Z}}(f)$ on $\mathcal{M}$ has a specialization to the normal bundle $\sigma(\widehat{\mathcal{Z}}(f))$, which is an arithmetic divisor on $N_{\mathcal{Y}} \mathcal{M}$. The specialization is defined in such a way that the arithmetic degree of $\widehat{\mathcal{Z}}(f)$ along $\mathcal{Y} \rightarrow \mathcal{M}$ is equal to the arithmetic degree of $\sigma(\widehat{\mathcal{Z}}(f))$ along the zero section $\mathcal{Y} \rightarrow N_{\mathcal{Y}} \mathcal{M}$. What we are able to show, essentially, is that every component of the arithmetic divisor $\sigma(\widehat{\mathcal{Z}}(f))$ that meets the zero section improperly has $\sigma(\widehat{\boldsymbol{T}})$, the specialization of the cotautological bundle, as its associated line bundle. This implies that every component of $\widehat{\mathcal{Z}}(f)$ that meets $\mathcal{Y}$ improperly contributes the same quantity, $[\widehat{\boldsymbol{T}}: \mathcal{Y}]$, to the degree $[\widehat{\mathcal{Z}}(f): \mathcal{Y}]$. The contribution to the intersection of the remaining components can be read off from the degrees of the divisors $\widehat{\mathcal{Z}}_{0}(m, \mu)$ computed in $\S 4$, and the calculation of $[\widehat{\boldsymbol{T}}: \mathcal{Y}]$ quickly reduces to the Chowla-Selberg formula.

Finally, we remark that the proof of Theorem A makes essential use of the Bruinier-Yang calculation (following Schofer [Sch09]) of the values of $\Phi(f)$ at the points of $\mathcal{Y}(\mathbb{C})$. Because of the particular way in which the divergent integral defining $\Phi(f)$ is regularized, the Green function $\Phi(f)$ has a well-defined value even at points of divisor $\mathcal{Z}(f)$ along which $\Phi(f)$ has a logarithmic singularity. In other words, the Green function comes, by construction, with a discontinuous extension to all points of $\mathcal{M}(\mathbb{C})$. Hu's thesis sheds some light on this phenomenon, and the overregularized values of $\Phi(f)$ at the points of $\mathcal{Z}(f)$ will play an essential role in our calculation of improper intersections.

\subsection{Acknowledgements}

The authors thank Jan Bruinier, José Burgos Gil, Steve Kudla, and Tonghai Yang for helpful conversations and insights. They also thank the referees for useful comments.

\section{The GSpin Shimura variety and its special divisors}

\subsection{Preliminaries}

Let $R$ be a commutative ring. A quadratic space over $R$ is a projective $R$-module $V$ of finite rank, equipped with a homogenous function $Q: V \rightarrow R$ of degree 2 for which the symmetric pairing

$$
[x, y]=Q(x+y)-Q(x)-Q(y)
$$

is $R$-bilinear. We say that $V$ is self-dual if this pairing induces an isomorphism $V \stackrel{\simeq}{\rightarrow} \operatorname{Hom}_{R}(V, R)$. 


\section{Height PAIRINGs on orthogonal Shimura varieties}

The Clifford algebra $C(V)$ is the quotient of the tensor algebra $\otimes V$ by the ideal generated by elements of the form $x \otimes x-Q(x)$. The $R$-algebra $C(V)$ is generated by the image of the natural injection $V \rightarrow C(V)$, and the grading on $\otimes V$ induces a $\mathbb{Z} / 2 \mathbb{Z}$ grading

$$
C(V)=C^{+}(V) \oplus C^{-}(V) .
$$

The Clifford algebra $C(V)$ has the following universal property: given an associative algebra $B$ over $R$ and a map of $R$-modules $f: V \rightarrow B$ satisfying $f(x)^{2}=Q(x)$ for all $x \in V$, there is a unique map of $R$-algebras $\tilde{f}: C(V) \rightarrow B$ satisfying $\left.\tilde{f}\right|_{V}=f$. In particular, applying this property with $B=C(V)^{\mathrm{op}}$ and $f$ the natural inclusion of $V$ in $C(V)^{\mathrm{op}}$, we obtain a canonical anti-involution $*$ on $C(V)$ satisfying

$$
\left(x_{1} x_{2} \cdots x_{r-1} x_{r}\right)^{*}=x_{r} x_{r-1} \cdots x_{2} x_{1}
$$

for any $x_{1}, x_{2}, \ldots, x_{r-1}, x_{r} \in V$.

To a self-dual quadratic space $V$, we can attach the reductive group scheme $\operatorname{GSpin}(V)$ over $R$ with functor of points

$$
\operatorname{GSpin}(V)(S)=\left\{g \in C^{+}(V) \underset{S}{\times}: g V_{S} g^{-1}=V_{S}\right\}
$$

for any $R$-algebra $S$. Denote by $g \bullet v=g v g^{-1}$ the natural action of $\operatorname{GSpin}(V)$ on $V$. For any $R$-algebra $S$, the map $g \mapsto g^{*} g$ on $\operatorname{GSpin}(V)(S)$ takes values in $S^{\times}$[Bas74, 3.2.1], and so defines a canonical homomorphism of $R$-group schemes

$$
\nu: \operatorname{GSpin}(V) \rightarrow \mathbb{G}_{m, R},
$$

which we call the spinor norm.

Choose an element $\delta \in C^{+}(V)^{\times}$satisfying $\delta^{*}=-\delta$. We can use this element to define a symplectic pairing on $C(V)$ as follows (see [MP16, (1.6)]). The self-duality hypothesis on $V$ implies that $C(V)$ is a graded Azumaya algebra over $R$ in the sense of [Bas74, §2.3, Theorem]. In particular, there is a canonical reduced trace form $\operatorname{Trd}: C(V) \rightarrow R$, and the pairing $\left(z_{1}, z_{2}\right) \mapsto$ $\operatorname{Trd}\left(z_{1} z_{2}\right)$ is a perfect symmetric bilinear form on $C(V)$. The desired symplectic pairing on $C(V)$ is $\psi_{\delta}\left(z_{1}, z_{2}\right)=\operatorname{Trd}\left(z_{1} \delta z_{2}^{*}\right)$.

It is easily checked that

$$
\psi_{\delta}\left(g z_{1}, g z_{2}\right)=\nu(g) \psi_{\delta}\left(z_{1}, z_{2}\right)
$$

for any $R$-algebra $S, z_{1}, z_{2} \in C(V)_{S}$, and $g \in \operatorname{GSpin}(V)(S)$. In other words, the action of $\operatorname{GSpin}(V)$ on $C(V)$ by left multiplication realizes GSpin $(V)$ as a subgroup of the $R$-group scheme $\mathrm{GSp}_{\delta}$ of symplectic similitudes of $\left(C(V), \psi_{\delta}\right)$, and the symplectic similitude character restricts to the spinor norm on $\operatorname{GSpin}(V)$.

\subsection{The complex Shimura variety}

Let $n \geqslant 1$ be an integer. Fix a quadratic space $(L, Q)$ over $\mathbb{Z}$ of signature $(n, 2)$, and set $V=L_{\mathbb{Q}}$. We assume throughout that $L$ is maximal in the sense that there is no lattice $L \subsetneq L^{\prime} \subset V$ satisfying $Q\left(L^{\prime}\right) \subset \mathbb{Z}$. Set $G=\operatorname{GSpin}(V)$; this is a reductive group over $\mathbb{Q}$.

Let $\mathcal{D}$ be the space of oriented negative 2-planes $\boldsymbol{h} \subset V_{\mathbb{R}}$. If we extend the $\mathbb{Q}$-bilinear form on $V$ to a $\mathbb{C}$-bilinear form on $V_{\mathbb{C}}$, the real manifold $\mathcal{D}$ is isomorphic to the Grassmannian

$$
\mathcal{D} \stackrel{\simeq}{\rightarrow}\left\{z \in V_{\mathbb{C}} \backslash\{0\}:[z, z]=0 \text { and }[z, \bar{z}]<0\right\} / \mathbb{C}^{\times},
$$

and in particular is naturally a complex manifold. The isomorphism is as follows: for each $\boldsymbol{h}$ pick an oriented basis $\{x, y\}$ so that $Q(x)=Q(y)$ and $[x, y]=0$, and let $z=x+i y$. The inverse 
construction is obvious.

There are canonical inclusions of $\mathbb{R}$-algebras $\mathbb{C} \hookrightarrow C(\boldsymbol{h}) \hookrightarrow C\left(V_{\mathbb{R}}\right)$, where the first is determined by

$$
i \mapsto \frac{x y}{\sqrt{Q(x) Q(y)}}
$$

The induced map $\mathbb{C}^{\times} \rightarrow C\left(V_{\mathbb{R}}\right)^{\times}$takes values in $G(\mathbb{R})$, and arises from a morphism of real algebraic groups

$$
\alpha_{\boldsymbol{h}}: \operatorname{Res}_{\mathbb{C} / \mathbb{R}} \mathbb{G}_{m} \rightarrow G_{\mathbb{R}}
$$

In this way we identify $\mathcal{D}$ with a $G(\mathbb{R})$-conjugacy class in $\operatorname{Hom}\left(\operatorname{Res}_{\mathbb{C} / \mathbb{R}} \mathbb{G}_{m}, G_{\mathbb{R}}\right)$.

Set $\widehat{L}=L_{\widehat{\mathbb{Z}}}$, and define a compact open subgroup

$$
K=G\left(\mathbb{A}_{f}\right) \cap C(\widehat{L})^{\times}
$$

of $G\left(\mathbb{A}_{f}\right)$. Note that $g \bullet \widehat{L}=\widehat{L}$ for all $g \in K$. Denote by

$$
L^{\vee}=\{x \in V:[x, L] \subset \mathbb{Z}\}
$$

the dual lattice of $L$. As in the proof of [MP16, (2.6)], the action of $K$ on $\widehat{L}$ defines a homomorphism $K \rightarrow \mathrm{SO}(\widehat{L})$ whose image is precisely the subgroup of elements acting trivially on the discriminant group $L^{\vee} / L \stackrel{\simeq}{\rightarrow} \widehat{L}^{\vee} / \widehat{L}$.

The pair $(G, \mathcal{D})$ is a Shimura datum, and

$$
M(\mathbb{C})=G(\mathbb{Q}) \backslash \mathcal{D} \times G\left(\mathbb{A}_{f}\right) / K \stackrel{\simeq}{\rightarrow} \bigsqcup_{g \in G(\mathbb{Q}) \backslash G\left(\mathbb{A}_{f}\right) / K} \Gamma_{g} \backslash \mathcal{D},
$$

is the complex orbifold of $\mathbb{C}$-points of the Shimura variety attached to this datum and level subgroup $K$. Here we have set $\Gamma_{g}=G(\mathbb{Q}) \cap g K g^{-1}$. We will refer to $M(\mathbb{C})$ as the GSpin Shimura variety associated with $L$.

Given an algebraic representation $G \rightarrow \underline{\operatorname{Aut}}(N)$ on a finite dimensional $\mathbb{Q}$-vector space, and a $K$-stable lattice $N_{\widehat{\mathbb{Z}}} \subset N_{\mathbb{A}_{f}}$, we obtain a $\mathbb{Z}$-local system $\boldsymbol{N}_{\mathrm{Be}}$ on $M(\mathbb{C})$ whose fiber at a point $[(\boldsymbol{h}, g)] \in M(\mathbb{C})$ is identified with $N \cap g N_{\widehat{\mathbb{Z}}}$. The corresponding vector bundle

$$
\boldsymbol{N}_{\mathrm{dR}, M(\mathbb{C})}=\mathcal{O}_{M(\mathbb{C})} \otimes \boldsymbol{N}_{\mathrm{Be}}
$$

is equipped with a filtration $\mathrm{Fil}^{\bullet} \boldsymbol{N}_{\mathrm{dR}, M(\mathbb{C})}$, which at any point $[(\boldsymbol{h}, g)]$ equips the fiber of $\boldsymbol{N}_{\mathrm{Be}}$ with the Hodge structure determined by (2.2). This gives us a functorial assignment from pairs $\left(N, N_{\widehat{\mathbb{Z}}}\right)$ as above to variations of $\mathbb{Z}$-Hodge structures over $M(\mathbb{C})$.

Applying this to $V$ and the lattice $\widehat{L} \subset V_{\mathbb{A}_{f}}$, we obtain a canonical variation of polarized $\mathbb{Z}$ Hodge structures $\left(\boldsymbol{V}_{\mathrm{Be}}, \mathrm{Fil}^{\bullet} \boldsymbol{V}_{\mathrm{dR}, M(\mathbb{C})}\right)$. For each point $z$ of (2.1) the induced Hodge decomposition of $V_{\mathbb{C}}$ has

$$
V_{\mathbb{C}}^{(1,-1)}=\mathbb{C} z, \quad V_{\mathbb{C}}^{(-1,1)}=\mathbb{C} \bar{z}, \quad V_{\mathbb{C}}^{(0,0)}=(\mathbb{C} z+\mathbb{C} \bar{z})^{\perp} .
$$

It follows that $\mathrm{Fil}^{1} \boldsymbol{V}_{\mathrm{dR}, M(\mathbb{C})}$ is an isotropic line and $\mathrm{Fil}^{0} \boldsymbol{V}_{\mathrm{dR}, M(\mathbb{C})}$ is its annihilator with respect to the pairing on $\boldsymbol{V}_{\mathrm{dR}, M(\mathbb{C})}$ induced from that on $L$.

Similarly, viewing $H=C(V)$ as a representation of $G$ by left multiplication, with its lattice $H_{\widehat{\mathbb{Z}}}=C(L)_{\widehat{\mathbb{Z}}}$, we obtain a variation of $\mathbb{Z}$-Hodge structures $\left(\boldsymbol{H}_{\mathrm{Be}}, \mathrm{Fil} \boldsymbol{H}^{\bullet} \boldsymbol{H}_{\mathrm{dR}, M(\mathbb{C})}\right)$. This variation has type $(-1,0),(0,-1)$ and is therefore the homology of a family of complex tori over $M(\mathbb{C})$.

This variation of $\mathbb{Z}$-Hodge structures is polarizable, and so the family of complex tori in fact arises from an abelian scheme over $M(\mathbb{C})$. To see this, first consider the representation of $G$ on $\mathbb{Q}$ 


\section{Height PAIRINGs on ORTHOgOnAL Shimura VARIETIES}

afforded by the spinor norm $\nu$, along with the obvious $K$-stable lattice $\widehat{\mathbb{Z}} \subset \mathbb{A}_{f}$. For any $\boldsymbol{h} \in \mathcal{D}$, the composition $\nu \circ \alpha_{\boldsymbol{h}}$ is the homomorphism

$$
\operatorname{Res}_{\mathbb{C} / \mathbb{R}} \mathbb{G}_{m} \stackrel{z \mapsto z \bar{z}}{\longrightarrow} \mathbb{G}_{m, \mathbb{R}} .
$$

From this, we see that the associated variation of $\mathbb{Z}$-Hodge structures is the Tate twist $\underline{\mathbb{Z}}(1)$, whose underlying $\mathbb{Z}$-local system is just the constant sheaf $\underline{\mathbb{Z}}$, but whose (constant) Hodge filtration is concentrated in degree -1 .

Now, choose $\delta \in C^{+}(L) \cap C^{+}(V)^{\times}$satisfying $\delta^{*}=-\delta$. As explained in (2.1), this defines a $G$-equivariant symplectic pairing

$$
\psi_{\delta}: C(V) \times C(V) \rightarrow \mathbb{Q}(\nu)
$$

by $\psi_{\delta}(x, y)=\operatorname{Trd}\left(x \delta y^{*}\right)$. Since $\delta$ lies in $C^{+}(L)$, the restriction of this form to $C(L)$ is $\mathbb{Z}$-valued, and in particular induces a $K$-invariant alternating form on $C(L)_{\widehat{\mathbb{Z}}}$ with values in $\widehat{\mathbb{Z}}(\nu)$.

We can arrange our choice of $\delta$ to have the following positivity property: For every $\boldsymbol{h} \in \mathcal{D}$, the Hermitian form $\psi_{\delta}\left(\alpha_{\boldsymbol{h}}(i) x, \bar{y}\right)$ on $C(V)_{\mathbb{C}}$ is (positive or negative) definite. To produce a concrete such choice, choose a negative definite plane $\boldsymbol{h}_{0} \subset V$ defined over $\mathbb{Q}$, and an oriented basis $x, y \in \boldsymbol{h}_{0} \cap L$ for $\boldsymbol{h}_{0}$ satisfying $[x, y]=0$. Then it can be checked that $\delta=x y \in C(L)$ has the desired positivity property. Any such choice of $\delta$ produces an alternating morphism

$$
\boldsymbol{H}_{\mathrm{Be}} \otimes \boldsymbol{H}_{\mathrm{Be}} \rightarrow \underline{\mathbb{Z}}(1)
$$

of variations of Hodge structures, and the positivity property implies that this is a polarization.

Remark 2.2.1. By our construction, any choice of $\delta \in C^{+}(L) \cap C(V)^{\times}$with the required positivity property produces a polarization on $\mathcal{A}_{M(\mathbb{C})}$. While the polarization on $\mathcal{A}_{M(\mathbb{C})}$ depends on the choice of $\delta$, the abelian scheme itself does not.

In sum, we have produced an abelian scheme $\mathcal{A}_{M(\mathbb{C})} \rightarrow M(\mathbb{C})$, whose homology is canonically identified with the polarizable variation of Hodge structures $\left(\boldsymbol{H}_{\mathrm{Be}}, \boldsymbol{H}_{\mathrm{dR}, M(\mathbb{C})}\right)$. We call this the Kuga-Satake abelian scheme. It has relative dimension $2^{n+1}$.

The action of $G$ on $H$ commutes with the right multiplication action of $C(V)$, and also respects the $\mathbb{Z} / 2 \mathbb{Z}$-grading $H=H^{+} \times H^{-}$defined by $H^{ \pm}=C^{ \pm}(V)$. Thus the Kuga-Satake abelian scheme inherits a right action of $C(L)$, and a $\mathbb{Z} / 2 \mathbb{Z}$-grading

$$
\mathcal{A}_{M(\mathbb{C})}=\mathcal{A}_{M(\mathbb{C})}^{+} \times \mathcal{A}_{M(\mathbb{C})}^{-} .
$$

Elements of $C^{+}(L)$ and $C^{-}(L)$ act as homogeneous endomorphisms of graded degrees zero and one, respectively. The left multiplication action of $V \subset C(V)$ on $H$ induces an embedding

$$
\left(\boldsymbol{V}_{\mathrm{Be}}, \operatorname{Fil} \boldsymbol{l}^{\bullet} \boldsymbol{V}_{\mathrm{dR}, M(\mathbb{C})}\right) \subset\left(\underline{\operatorname{End}}\left(\boldsymbol{H}_{\mathrm{Be}}\right), \operatorname{Fil}{ }^{\bullet} \underline{\operatorname{End}}\left(\boldsymbol{H}_{\mathrm{dR}, M(\mathbb{C})}\right)\right)
$$

of variations of Hodge structures.

For $x \in V$ of positive length, define a divisor on $\mathcal{D}$ by

$$
\mathcal{D}(x)=\{z \in \mathcal{D}: z \perp x\} .
$$

As in the work of Borcherds [Bor98], Bruinier [Bru02], and Kudla [Kud04], for every $m \in \mathbb{Q}_{>0}$ and $\mu \in L^{\vee} / L$ we define a complex orbifold

$$
Z(m, \mu)(\mathbb{C})=\bigsqcup_{g \in G(\mathbb{Q}) \backslash G\left(\mathbb{A}_{f}\right) / K} \Gamma_{g} \backslash\left(\bigsqcup_{\begin{array}{c}
x \in \mu_{g}+L_{g} \\
Q(x)=m
\end{array}} \mathcal{D}(x)\right) .
$$




\section{F. Andreatta, E. Z. Goren, B. Howard, K. Madapusi Pera}

Here $L_{g} \subset V$ is the $\mathbb{Z}$-lattice determined by $\widehat{L}_{g}=g \bullet \widehat{L}$, and $\mu_{g}=g \bullet \mu \in L_{g}^{\vee} / L_{g}$. Comparing with (2.3), the natural finite and unramified morphism

$$
Z(m, \mu)(\mathbb{C}) \rightarrow M(\mathbb{C})
$$

allows us to view (2.4) as an effective Cartier divisor on $M(\mathbb{C})$. We will see below that (2.4) admits a moduli interpretation in terms of the Kuga-Satake abelian scheme.

\subsection{Canonical models over $\mathbb{Q}$}

The reflex field of the Shimura datum $(G, \mathcal{D})$ is $\mathbb{Q}$, and hence $M(\mathbb{C})$ is the complex fiber of an algebraic stack $M$ over $\mathbb{Q}$. By [MP16, §3] the Kuga-Satake abelian scheme, along with all its additional structures, has a canonical descent $\pi: \mathcal{A}_{M} \rightarrow M$.

We denote by ${ }^{1}$

$$
\boldsymbol{H}_{\mathrm{dR}, M}=\underline{\operatorname{Hom}}\left(R^{1} \pi_{\mathrm{Zar}, *} \Omega_{\mathcal{A}_{M} / M}^{\bullet}, \mathcal{O}_{M}\right)
$$

the first de Rham homology of $\mathcal{A}_{M}$ relative to $M$; that is, the dual of the first relative hypercohomology of the de Rham complex. It is a locally free $\mathcal{O}_{M}$-module of rank $2^{n+2}$, equipped with its Hodge filtration $\mathrm{Fil}^{0} \boldsymbol{H}_{\mathrm{dR}, M} \subset \boldsymbol{H}_{\mathrm{dR}, M}$ and Gauss-Manin connection. It also has a $\mathbb{Z} / 2 \mathbb{Z}$-grading $\boldsymbol{H}_{\mathrm{dR}, M}=\boldsymbol{H}_{\mathrm{dR}, M}^{+} \times \boldsymbol{H}_{\mathrm{dR}, M}^{-}$arising from the grading $\mathcal{A}_{M}=\mathcal{A}_{M}^{+} \times \mathcal{A}_{M}^{-}$on the Kuga-Satake abelian scheme, and similarly a right action of $C(L)$ induced from that on $\mathcal{A}_{M}$. Over $M(\mathbb{C})$, it is canonically identified with $\boldsymbol{H}_{\mathrm{dR}, M(\mathbb{C})}$ with its filtration and integrable connection.

From [MP16, (3.12)], we find that there is a canonical descent $\boldsymbol{V}_{\mathrm{dR}, M}$ of $\boldsymbol{V}_{\mathrm{dR}, M(\mathbb{C})}$, along with its filtration and integrable connection, so that we have an embedding

$$
\boldsymbol{V}_{\mathrm{dR}, M} \subset \underline{\operatorname{End}}_{C(L)}\left(\boldsymbol{H}_{\mathrm{dR}, M}\right)
$$

of filtered vector bundles over $M$ with integrable connection. This embedding is a descent of the one already available over $M(\mathbb{C})$. There is a canonical quadratic form $\boldsymbol{Q}: \boldsymbol{V}_{\mathrm{dR}, M} \rightarrow \mathcal{O}_{M}$ given on sections by $x \circ x=\boldsymbol{Q}(x) \cdot \mathrm{Id}$, where the composition takes place in $\underline{\operatorname{End}}_{C(L)}\left(\boldsymbol{H}_{\mathrm{dR}, M}\right)$.

In the same way we define

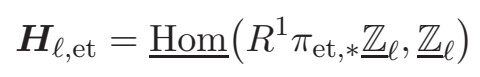

to be the first $\ell$-adic étale homology of $\mathcal{A}_{M}$ relative to $M$. Over $M(\mathbb{C})$, it is canonically identified with $\mathbb{Z}_{\ell} \otimes \boldsymbol{H}_{\mathrm{Be}}$. The local system $\mathbb{Z}_{\ell} \otimes \boldsymbol{V}_{\mathrm{Be}}$ descends to an $\ell$-adic sheaf $\boldsymbol{V}_{\ell, \text { et }}$ over $M$, and there is a canonical embedding as a local direct summand

$$
\boldsymbol{V}_{\ell, \text { et }} \subset \underline{\operatorname{End}}_{C(L)}\left(\boldsymbol{H}_{\ell, \text { et }}\right) .
$$

Again, composition on the right hand side induces a quadratic form on $\boldsymbol{V}_{\ell \text {,et }}$ with values in the constant sheaf $\underline{\mathbb{Z}}_{\ell}$.

\subsection{Integral models}

The stack $M$ admits a regular integral model $\mathcal{M}$ over $\mathbb{Z}$, which we construct in this section following [MP16, §7]. In loc. cit. such a model is constructed over $\mathbb{Z}[1 / 2]$ as one uses the main result of [Kis10], where 2 is assumed to be invertible. As we shall see, most of the results extend also to the case of the prime $p=2$ replacing [Kis10] with [KM15]. The construction is involved, and the final definition of $\mathcal{M}$ is given in Proposition 2.4.6.

\footnotetext{
${ }^{1}$ In [MP16], one works consistently with cohomology rather than homology, and so the conventions here will be dual to the conventions there.
} 


\section{Height Pairings on orthogonal Shimura varieties}

It follows from [MP16, (6.8)] that we may fix an isometric embedding $L \subset L^{\diamond}$, where $\left(L^{\diamond}, Q^{\diamond}\right)$ is a maximal quadratic space over $\mathbb{Z}$, self-dual at $p$ and of signature $\left(n^{\diamond}, 2\right)$. Set

$$
\Lambda=L^{\perp}=\left\{x \in L^{\diamond}: x \perp L\right\} .
$$

The construction of the integral model over $\mathbb{Z}_{(p)}$ of $M$ will proceed by first constructing a smooth integral model for the larger Shimura variety $M^{\diamond}$ associated with $L^{\diamond}$. There is a finite and unramified morphism $M \rightarrow M^{\diamond}$ over $\mathbb{Q}$ which we will extend to integral models, and the integral model for $M$ will inherit extra structure (for example, the vector bundles of Proposition 2.4.5 below) realized by pulling back structures from the larger integral model.

Over $M^{\diamond}$ we have the vector bundle $\boldsymbol{V}_{\mathrm{dR}, M^{\diamond}}$ associated with $L^{\diamond}$. Let $\boldsymbol{V}_{\mathrm{dR}, M}^{\diamond}$ be its restriction to $M$. By construction, we have an isometric embedding

$$
\boldsymbol{V}_{\mathrm{dR}, M} \hookrightarrow \boldsymbol{V}_{\mathrm{dR}, M}^{\diamond}
$$

of filtered vector bundles with integrable connections over $M$. Setting $\boldsymbol{\Lambda}_{\mathrm{dR}, M}=\Lambda \otimes_{\mathbb{Z}} \mathcal{O}_{M}$, we obtain an injection of vector bundles

$$
\boldsymbol{\Lambda}_{\mathrm{dR}, M} \hookrightarrow \boldsymbol{V}_{\mathrm{dR}, M}^{\diamond}
$$

identifying $\boldsymbol{\Lambda}_{\mathrm{dR}, M}$ with a local direct summand of $\boldsymbol{V}_{\mathrm{dR}, M}^{\diamond}$.

The Shimura variety $M^{\diamond}$ admits a smooth canonical model $\mathcal{M}_{\mathbb{Z}_{(p)}}^{\diamond}$ over $\mathbb{Z}_{(p)}$. This is the main theorem of [Kis10] for $p \neq 2$ and it is proven in general in [KM15]. We now recall the construction. Choose $\delta \in C^{+}\left(L^{\diamond}\right) \cap C\left(V^{\diamond}\right)^{\times}$as in $\S 2.2$, so that the associated symplectic pairing $\psi_{\delta}$ on $C\left(L^{\diamond}\right)$ produces a polarization on the Kuga-Satake abelian scheme

$$
A_{M \diamond(\mathbb{C})}^{\diamond} \rightarrow M^{\diamond}(\mathbb{C}) .
$$

This polarization has degree $d^{\diamond}$, where $d^{\diamond}$ is the discriminant of the restriction of $\psi_{\delta}$ to $C\left(L^{\diamond}\right)$. The polarized Kuga-Satake abelian scheme defines a morphism of complex algebraic stacks

$$
i_{\delta}: M_{\mathbb{C}}^{\diamond} \rightarrow \mathcal{X}_{2^{n^{\diamond}+1}, d^{\diamond}, \mathbb{C}}^{\text {Siegel }}
$$

where $\mathcal{X}_{2^{n^{\diamond}+1}, d^{\diamond}}^{\text {Siegel }}$ is the Siegel moduli stack over $\mathbb{Z}$ parameterizing polarized abelian schemes of dimension $2^{n^{\diamond}+1}$ of degree $d^{\diamond}$. It can be checked using complex uniformization that this map is finite and unramified. The theory of canonical models of Shimura varieties implies that $i_{\delta}$ descends to a finite and unramified map of $\mathbb{Q}$-stacks

$$
i_{\delta}: M^{\diamond} \rightarrow \mathcal{X}_{2^{n \diamond+1}, d^{\diamond}, \mathbb{Q}}^{\text {Siegel }}
$$

The integral model $\mathcal{M}_{\mathbb{Z}_{(p)}}^{\diamond}$ is defined as the normalization of $\mathcal{X}_{2^{n^{\diamond}+1}, d^{\diamond}, \mathbb{Z}_{(p)}}^{\text {Siegel }}$ in $M^{\diamond}$, in the following sense:

Definition 2.4.1. Given an algebraic stack $\mathcal{X}$ over $\mathbb{Z}_{(p)}$, and a normal algebraic stack $Y$ over $\mathbb{Q}$ equipped with a finite map $j_{\mathbb{Q}}: Y \rightarrow \mathcal{X}_{\mathbb{Q}}$, the normalization of $\mathcal{X}$ in $Y$ is the finite $\mathcal{X}$-stack $j: \mathcal{Y} \rightarrow \mathcal{X}$, characterized by the property that $j_{*} \mathcal{O}_{\mathcal{Y}}$ is the integral closure of $\mathcal{O}_{\mathcal{X}}$ in $\left(j_{\mathbb{Q}}\right)_{*} \mathcal{O}_{Y}$.

The normalization is uniquely determined by the following universal property: given a finite

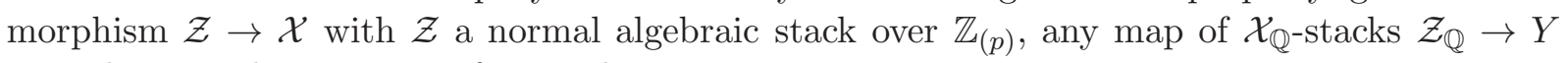
extends uniquely to a map of $\mathcal{X}$-stacks $\mathcal{Z} \rightarrow \mathcal{Y}$.

Proposition 2.4.2. The stack $\mathcal{M}_{\mathbb{Z}_{(p)}}^{\diamond}$ is smooth over $\mathbb{Z}_{(p)}$. It does not depend on the choice of $\delta \in C^{+}\left(L^{\diamond}\right) \cap C\left(V^{\diamond}\right)^{\times}$. 


\section{F. Andreatta, E. Z. Goren, B. Howard, K. Madapusi Pera}

Proof. This essentially follows from [Kis10, (2.3.8)] if $p \neq 2$ and from [KM15, Theorem 3.10] in general. We provide some of the details.

Abbreviate

$$
\mathcal{X}^{\text {Siegel }}=\mathcal{X}_{2^{n \diamond+1}, d^{\diamond}, \mathbb{Z}_{(p)}}^{\text {Siegl }}
$$

and let $\left(\mathcal{A}^{\text {Siegel }}, \lambda^{\text {Siegel }}\right)$ be the universal polarized abelian scheme over $\mathcal{X}^{\text {Siegel }}$. For any $m \in \mathbb{Z}_{>0}$ coprime to $p d^{\diamond}$, let $\mathcal{X}_{m}^{\text {Siegel }} \rightarrow \mathcal{X}^{\text {Siegel }}$ be the finite étale cover parameterizing full level- $m$ structures on $\mathcal{A}^{\text {Siegel}}$; that is, parametrizing isomorphisms of group schemes

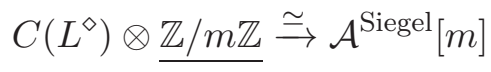

that carry the symplectic form $\psi_{\delta} \otimes 1$ on the left hand side to a $(\mathbb{Z} / m \mathbb{Z})^{\times}$-multiple of the Weil pairing on $\mathcal{A}^{\text {Siegel }}[m]$ induced by $\lambda^{\text {Siegel }}$. For $m \geqslant 3$ the stack $\mathcal{X}_{m}^{\text {Siegel }}$ is a scheme over $\mathbb{Z}_{(p)}$.

Write $G^{\diamond}=\operatorname{GSpin}\left(V^{\diamond}\right)$, and set

$$
K^{\diamond}=G^{\diamond}\left(\mathbb{A}_{f}\right) \cap C\left(L^{\diamond}\right)_{\widehat{\mathbb{Z}}}^{\times}
$$

Let $K^{\diamond}(m) \subset K^{\diamond}$ be the largest subgroup acting trivially on $C\left(L^{\diamond}\right) \otimes(\mathbb{Z} / m \mathbb{Z})$. We obtain a finite étale cover $M_{m}^{\diamond} \rightarrow M^{\diamond}$ of Shimura varieties with Galois group $K^{\diamond} / K^{\diamond}(m)$, where

$$
M_{m}^{\diamond}(\mathbb{C})=G^{\diamond}(\mathbb{Q}) \backslash \mathcal{D}^{\diamond} \times G^{\diamond}\left(\mathbb{A}_{f}\right) / K^{\diamond}(m)
$$

and $\mathcal{D}^{\diamond}$ is the space of oriented negative definite planes in $V_{\mathbb{R}}^{\diamond}$.

The map $i_{\delta}: M^{\diamond} \rightarrow \mathcal{X}_{\mathbb{Q}}^{\text {Siegel }}$ lifts to a map

$$
i_{\delta, m}: M_{m}^{\diamond} \rightarrow \mathcal{X}_{m, \mathbb{Q}}^{\text {Siegel }} .
$$

From $\left[\right.$ Kis10, (2.1.2)] we find that $i_{\delta, m}$ is a closed immersion of $\mathbb{Q}$-schemes for sufficiently large $m$. Fix such an $m$, and let $\mathcal{M}_{m, \mathbb{Z}_{(p)}}^{\diamond}$ be the normalization of $\mathcal{X}_{m}^{\text {Siegel }}$ in $M_{m}^{\diamond}$. Since $i_{\delta, m}$ is a closed immersion, this is simply the normalization of the Zariski closure of $M_{m}^{\diamond}$ in $\mathcal{X}_{m}^{\text {Siegel }}$. It now follows from $\left[\right.$ Kis10, (2.3.8)] and [KM15, Theorem 3.10] that $\mathcal{M}_{m, \mathbb{Z}_{(p)}}$ is smooth over $\mathbb{Z}_{(p)}$, and moreover, that it is, up to unique isomorphism, independent of the choice of $\delta$.

The stack $\mathcal{M}_{\mathbb{Z}_{(p)}}^{\diamond}$ is the étale quotient of $\mathcal{M}_{m, \mathbb{Z}_{(p)}}^{\diamond}$ by the action of $K^{\diamond} / K^{\diamond}(m)$, and so the proposition follows.

By the construction of the integral model $\mathcal{M}_{\mathbb{Z}_{(p)}}^{\diamond}$, the Kuga-Satake abelian scheme $A^{\diamond} \rightarrow M^{\diamond}$ extends to $\mathcal{A}^{\diamond} \rightarrow \mathcal{M}_{\mathbb{Z}_{(p)}}$. A non-trivial consequence of the construction is that the vector bundle $\boldsymbol{V}_{\mathrm{dR}, M^{\diamond}}^{\diamond}$, with its integrable connection and filtration, extends to a filtered vector bundle with integrable connection $\left(\boldsymbol{V}_{\mathrm{dR}, \mathcal{M}_{\mathbb{Z}}^{\diamond}(p)}^{\diamond}, \mathrm{Fil}^{\bullet} \boldsymbol{V}_{\mathrm{dR}, \mathcal{M}_{\mathbb{Z}_{(p)}}^{\diamond}}\right)$ over $\mathcal{M}_{\mathbb{Z}_{(p)}}^{\diamond}$ (see [MP16, §4] for details).

Let $\boldsymbol{H}_{\mathrm{dR}, \mathcal{M}_{\mathbb{Z}}^{\diamond}}^{\diamond}$ be the first de Rham homology of $\mathcal{A}^{\diamond}$, so that we have an embedding of filtered vector bundles

$$
\boldsymbol{V}_{\mathrm{dR}, \mathcal{M}_{\mathbb{Z}_{(p)}}^{\diamond}}^{\diamond} \underline{\operatorname{End}}_{C(L)}\left(\boldsymbol{H}_{\mathrm{dR}, \mathcal{M}_{\mathbb{Z}_{(p)}}^{\diamond}}^{\diamond}\right)
$$

extending that in the generic fiber and exhibiting its source as a local direct summand of the target. Composition in the endomorphism ring gives a non-degenerate quadratic form on

$$
\boldsymbol{Q}^{\diamond}: \boldsymbol{V}_{\mathrm{dR}, \mathcal{M}_{\mathbb{Z}}^{\diamond}}^{\diamond} \rightarrow \mathcal{O}_{\mathcal{M}_{\mathbb{Z}_{(p)}}^{\diamond}}
$$

again extending that on its generic fiber. 
Similarly, let

$$
\boldsymbol{H}_{\mathrm{crys}}^{\diamond}=\underline{\operatorname{Hom}}\left(R^{1} \pi_{\mathrm{crys}, *} \mathcal{O}_{\mathcal{A}_{\mathbb{F}} / \mathbb{Z}_{p}}^{\text {crys }}, \mathcal{O}_{\mathcal{M}_{\mathbb{F}}}^{\text {crys }} / \mathbb{Z}_{p}\right)
$$

be the first crystalline homology of $\mathcal{A}_{\mathbb{F}_{p}}^{\diamond}$ relative to $\mathcal{M}_{\mathbb{F}_{p}}^{\diamond}$. As $\mathcal{M}_{\mathbb{Z}_{(p)}}^{\diamond}$ is smooth over $\mathbb{Z}_{(p)}$, the vector bundle with integrable connection (2.6) determines a crystal $\boldsymbol{V}_{\text {crys }}^{\diamond}$ over $\mathcal{M}_{\mathbb{F}_{p}}^{\diamond}$ equipped with an embedding

$$
\boldsymbol{V}_{\text {crys }}^{\diamond} \subset \underline{\operatorname{End}}_{C(L)}\left(\boldsymbol{H}_{\text {crys }}^{\diamond}\right)
$$

as a local direct summand; see [MP16, (4.14)] for details.

We are now ready to give the construction of the desired integral model $\mathcal{M}_{\mathbb{Z}_{(p)}}$ for $M$. The isometric embedding $V \rightarrow V^{\diamond}$ induces a homomorphism of Clifford algebras, which restricts to a closed immersion $G \rightarrow G^{\diamond}$. This induces a morphism of Shimura varieties $M \rightarrow M^{\diamond}$. The direct summands $\Lambda_{\mathbb{Q}} \subset V^{\diamond}$ and $\Lambda_{\widehat{\mathbb{Z}}} \subset L_{\widehat{\mathbb{Z}}}^{\diamond}$ are pointwise stabilized by $G(\mathbb{Q})$ and $K$, respectively. From this, arguing as in $[\mathrm{MP} 16,(6.5)]$, we find that there is a canonical embedding

$$
\Lambda \subset \operatorname{End}_{C\left(L^{\diamond}\right)}\left(\left.\mathcal{A}^{\diamond}\right|_{M}\right)
$$

whose de Rham realization is $(2.5)$. Let $\check{\mathcal{M}}_{\mathbb{Z}_{(p)}}$ be the normalization of $\mathcal{M}_{\mathbb{Z}_{(p)}}$ in $M$. Then, by [FC90, Prop. 2.7], the embedding (2.7) extends to

$$
\Lambda \subset \operatorname{End}_{C\left(L^{\diamond}\right)}\left(\left.\mathcal{A}^{\diamond}\right|_{\check{\mathcal{M}}_{\mathbb{Z}_{(p)}}}\right) \text {. }
$$

Since $\boldsymbol{V}_{\mathrm{dR}, \mathcal{M}_{\mathbb{Z}}^{\diamond}}^{\diamond}$ is a direct summand of $\underline{\operatorname{End}}_{C(L)}\left(\boldsymbol{H}_{\mathrm{dR}, \mathcal{M}_{\mathbb{Z}_{(p)}}^{\diamond}}\right)$, the de Rham realization of (2.8) provides us with an extension

of $(2.5)$, where

$$
\boldsymbol{\Lambda}_{\mathrm{dR}} \rightarrow \boldsymbol{V}_{\mathrm{dR}, \check{\mathcal{M}}_{\mathbb{Z}_{(p)}}}
$$

$$
\Lambda_{\mathrm{dR}}=\Lambda \otimes_{\mathbb{Z}} \mathcal{O}_{\check{\mathcal{M}}_{\mathbb{Z}_{(p)}}}
$$

Let $\check{\mathcal{M}}_{\mathbb{Z}_{(p)}}^{\text {pr }} \subset \check{\mathcal{M}}_{\mathbb{Z}_{(p)}}$ be the open locus where the cokernel of (2.9) is a vector bundle of rank $n$ (see [MP16, 6.16(i)]). This is also the locus over which the image of (2.9) is a local direct summand. In particular, it contains the generic fiber $M$ of $\check{\mathcal{M}}_{\mathbb{Z}_{(p)}}$.

Let $\mathrm{M}^{\mathrm{loc}}(L)$ be the projective scheme over $\mathbb{Z}$ parameterizing isotropic lines $L^{1} \subset L$. The following can be deduced from [MP16, (6.16),(6.20),(2.16)].

Proposition 2.4.3.

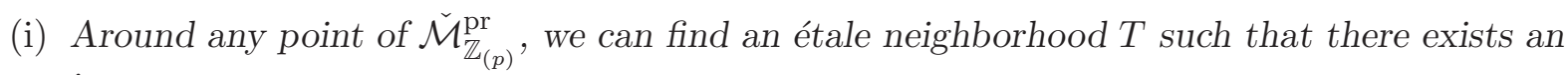
isometry

$$
\xi: \mathcal{O}_{T} \otimes L^{\diamond} \stackrel{\simeq}{\rightarrow} \boldsymbol{V}_{\mathrm{dR}, T}
$$

satisfying $\xi\left(\mathcal{O}_{T} \otimes \Lambda\right)=\boldsymbol{\Lambda}_{\mathrm{dR}, T}$, and such that the morphism $T \rightarrow \mathrm{M}^{\mathrm{loc}}(L)$ determined by the isotropic line

is étale.

$$
\xi^{-1}\left(\mathrm{Fil}^{1} \boldsymbol{V}_{\mathrm{dR}, T}^{\diamond}\right) \subset \mathcal{O}_{T} \otimes L^{\diamond}
$$

(ii) If $L_{(p)}$ is self-dual, or if $p>2$ and $p^{2} \nmid \operatorname{disc}(L)$, then $\check{\mathcal{M}}_{\mathbb{Z}_{(p)}}^{\mathrm{pr}}=\check{\mathcal{M}}_{\mathbb{Z}_{(p)}}$.

(iii) If $p>2$ and $p^{2} \mid \operatorname{disc}(L)$, then $\check{\mathcal{M}}_{\mathbb{Z}_{(p)}}^{\text {pr }}$ is regular outside of a 0 dimensional closed substack.

We now define: 


\section{F. Andreatta, E. Z. Goren, B. Howard, K. Madapusi Pera}

$-\mathcal{M}_{\mathbb{Z}_{(p)}}=\check{\mathcal{M}}_{\mathbb{Z}_{(p)}}$ if $L_{(p)}$ is self-dual, or if $p>2$ and $p^{2} \nmid \operatorname{disc}(L)$;

- $\mathcal{M}_{\mathbb{Z}_{(p)}}=$ the regular locus of $\check{\mathcal{M}}_{\mathbb{Z}_{(p)}}^{\text {pr }}$ if $p>2$ and $p^{2} \mid \operatorname{disc}(L)$;

$-\mathcal{M}_{\mathbb{Z}_{(p)}}=$ the smooth locus of $\check{\mathcal{M}}_{\mathbb{Z}_{(p)}}^{\text {pr }}$ if $p=2$.

Then $\mathcal{M}_{\mathbb{Z}_{(p)}}$ is the desired regular integral model for $M$ over $\mathbb{Z}_{(p)}$.

Remark 2.4.4. If $p=2$ and $L_{(2)}$ is not self-dual, or if $p>2$ and $p^{2} \mid \operatorname{disc}(L)$, this is certainly not the optimal definition of the regular model $\mathcal{M}_{\mathbb{Z}_{(p)}}$. For a discussion and examples of this when $p$ is odd, see [MP16, (6.27),(6.28)].

Equip $\Lambda_{\mathrm{dR}}=\Lambda \otimes_{\mathbb{Z}} \mathcal{O}_{\mathcal{M}_{\mathbb{Z}_{(p)}}}$ with the integrable connection $1 \otimes \mathrm{d}$, and the trivial filtration concentrated in degree 0 . By construction, over $\mathcal{M}_{\mathbb{Z}_{(p)}}$ we have a canonical isometric embedding

$$
\boldsymbol{\Lambda}_{\mathrm{dR}} \subset \boldsymbol{V}_{\mathrm{dR}, \mathcal{M}_{\mathbb{Z}_{(p)}}}
$$

of filtered vector bundles with integrable connections. Therefore, since the quadratic form on $\boldsymbol{V}_{\mathrm{dR}, \mathcal{M}_{\mathbb{Z}_{(p)}}}$ is self-dual, the orthogonal complement

$$
\boldsymbol{V}_{\mathrm{dR}, \mathcal{M}_{\mathbb{Z}_{(p)}}}=\boldsymbol{\Lambda}_{\mathrm{dR}}^{\perp} \subset \boldsymbol{V}_{\mathrm{dR}, \mathcal{M}_{\mathbb{Z}_{(p)}}}
$$

is again a vector sub-bundle with integrable connection. Indeed, it is precisely the kernel of the surjection of vector bundles

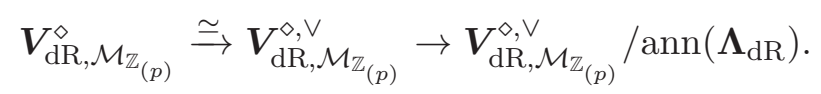

Moreover, the isotropic line $\mathrm{Fil}^{1} \boldsymbol{V}_{\mathrm{dR}, \mathcal{M}_{\mathbb{Z}_{(p)}}}$ is actually contained in $\boldsymbol{V}_{\mathrm{dR}, \mathcal{M}_{\mathbb{Z}_{(p)}}}$. For convenience, we abbreviate

$$
\boldsymbol{V}_{\mathrm{dR}, \mathcal{M}_{\mathbb{Z}_{(p)}}^{1}}=\mathrm{Fil}^{1} \boldsymbol{V}_{\mathrm{dR}, \mathcal{M}_{\mathbb{Z}_{(p)}}} .
$$

Proposition 2.4.5. The integral models $\mathcal{M}_{\mathbb{Z}_{(p)}}$ and $\check{\mathcal{M}}_{\mathbb{Z}_{(p)}}$ are, up to unique isomorphism, independent of the auxiliary choice of $L^{\diamond}$. Furthermore, the vector bundle $\boldsymbol{V}_{\mathrm{dR}, \mathcal{M}_{\mathbb{Z}_{(p)}}}$ and the

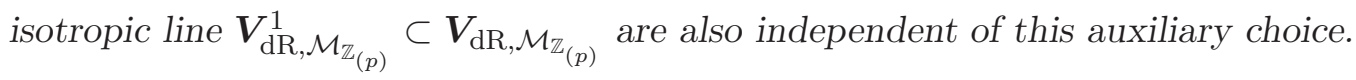

Proof. Suppose that we have a different embedding $L \hookrightarrow L_{1}^{\diamond}$ with $L_{1}^{\diamond}$ maximal, self-dual at $p$, and of signature $\left(n_{1}^{\diamond}, 2\right)$.

First, assume that the embedding $L \hookrightarrow L_{1}^{\diamond}$ factors as $L \hookrightarrow L^{\diamond} \hookrightarrow L_{1}^{\diamond}$. The construction of Kisin in [Kis10] and its generalization in [KM15] show that the map of integral models $\mathcal{M}_{\mathbb{Z}_{(p)}} \rightarrow \mathcal{M}_{1, \mathbb{Z}_{(p)}}$ associated with $L^{\diamond} \hookrightarrow L_{1}^{\diamond}$ is finite and unramified. Moreover, Proposition 2.4.3 shows that, if $\Lambda_{1}=\left(L^{\diamond}\right)^{\perp} \subset L_{1}^{\diamond}$, then the corresponding map

$$
\boldsymbol{\Lambda}_{1, \mathrm{dR}, \mathcal{M}_{\mathbb{Z}_{(p)}}^{\diamond}} \rightarrow \boldsymbol{V}_{1, \mathrm{dR}, \mathcal{M}_{\mathbb{Z}_{(p)}}}^{\diamond}
$$

is an embedding of vector bundles, and that its orthogonal complement is exactly $\boldsymbol{V}_{\mathrm{dR}, \mathcal{M}_{\mathbb{Z}_{(p)}}}$. From this, we easily deduce that the constructions of both $\check{\mathcal{M}}_{\mathbb{Z}_{(p)}}$ and $\check{\mathcal{M}}_{\mathbb{Z}_{(p)}}^{\text {pr }}$ do not depend on the choice of isometric embedding. The proof also shows that the vector bundle $\boldsymbol{V}_{\mathrm{dR}, \mathcal{M}_{\mathbb{Z}_{(p)}}}$, along with its isotropic line, is also independent of this choice.

For the general case, consider the embedding $\Delta: L \hookrightarrow L^{\diamond} \oplus L_{1}^{\diamond}$ defined by $v \mapsto(v,-v)$. The quadratic form on $L^{\diamond} \oplus L_{1}^{\diamond}$ given by the orthogonal sum of those on its individual summands 


\section{Height PAIRINGs on ORTHOgOnAL Shimura VARIETIES}

induces a quadratic form on the quotient

$$
L_{2}^{\diamond}=\left(L^{\diamond} \oplus L_{1}^{\diamond}\right) / \Delta(L)
$$

of signature $\left(n^{\diamond}+n_{1}^{\diamond}-n, 2\right)$. Moreover, the natural maps $L^{\diamond} \rightarrow L_{2}^{\diamond}$ and $L_{1}^{\diamond} \rightarrow L_{2}^{\diamond}$ are isometric inclusions of direct summands, whose restrictions to $L$ coincide.

Now, $L_{2}^{\diamond}$ need not be self-dual at $p$, but, via the argument in [MP16, (6.8)], we can embed it isometrically as a direct summand in a maximal quadratic space $L_{3}^{\diamond}$ of signature $\left(n_{3}^{\diamond}, 2\right)$, which is self-dual at $p$. In particular, replacing $L_{1}^{\diamond}$ with $L_{3}^{\diamond}$, we are reduced to the already considered case where $L^{\diamond}$ is an isometric direct summand of $L_{1}^{\diamond}$.

We can now give the construction of the stack $\mathcal{M}$ over $\mathbb{Z}$. It is simply obtained by patching together the spaces $\mathcal{M}_{\mathbb{Z}_{(p)}}$. To do this rigorously, we choose a finite collection of maximal quadratic spaces $L_{1}^{\diamond}, L_{2}^{\diamond}, \ldots, L_{r}^{\diamond}$ with the following properties:

- For each $i=1,2, \ldots, r, L_{i}^{\diamond}$ has signature $\left(n_{i}^{\diamond}, 2\right)$, for $n_{i}^{\diamond} \in \mathbb{Z}_{>0}$;

- For each $i$, there is an isometric embedding $L \hookrightarrow L_{i}^{\diamond}$;

- If, for each $i$, we denote by $S_{i}$ the set of primes dividing $\operatorname{disc}\left(L_{i}^{\diamond}\right)$, then $\bigcap_{i=1}^{r} S_{i}=\emptyset$.

It is always possible to find such a collection. For instance, first choose any maximal quadratic lattice of signature $\left(n_{1}^{\diamond}, 2\right)$, admitting $L$ as an isometric direct summand. Let $\left\{p_{2}, \ldots, p_{r}\right\}$ be the set of primes dividing the discriminant of $L_{1}^{\diamond}$. Now, for each $i=2,3, \ldots, r$, let $L_{i}^{\diamond}$ be a maximal quadratic lattice, self-dual at $p_{i}$, of signature $\left(n_{i}^{\diamond}, 2\right)$, admitting $L$ as an isometric direct summand. Then the collection $\left\{L_{1}^{\diamond}, \ldots, L_{r}^{\diamond}\right\}$ does the job.

For $i=1,2, \ldots, r$, let $M_{i}^{\diamond}$ be the GSpin Shimura variety over $\mathbb{Q}$ attached to $L_{i}^{\diamond}$. Given an appropriate choice of $\delta_{i} \in C^{+}\left(L_{i}^{\diamond}\right) \cap C\left(L_{i}^{\diamond}\right)_{\mathbb{Q}}^{\times}$, with associated alternating form $\psi_{\delta_{i}}$ on $C\left(L_{i}^{\diamond}\right)$, we obtain a sequence of finite and unramified map of $\mathbb{Q}$-stacks

$$
M \rightarrow M_{i}^{\diamond} \stackrel{i_{\delta_{i}}}{\longrightarrow} \mathcal{X}_{2^{n_{i}+1}, d_{i}^{\diamond}, \mathbb{Q}}^{\text {Siegel }}
$$

Here, $d_{i}^{\diamond}$ is the discriminant of $\psi_{\delta_{i}}$.

Let $\mathbb{Z}\left[\left(S_{i}\right)^{-1}\right] \subset \mathbb{Q}$ be the localization of $\mathbb{Z}$ obtained by inverting the primes in $S_{i}$. Let $\mathcal{M}_{\mathbb{Z}\left[\left(S_{i}\right)^{-1}\right]}$ be the stack over $\mathbb{Z}\left[\left(S_{i}\right)^{-1}\right]$ obtained by normalizing $\mathcal{X}_{2^{n_{i}^{\diamond}+1}, d_{i}^{\diamond}, \mathbb{Z}\left[\left(S_{i}\right)^{-1}\right]}^{\text {Siegel }} M$.

Proposition 2.4.6. There is a regular, flat, algebraic $\mathbb{Z}$-stack $\mathcal{M}$ such that for each $i$ the restriction of $\mathcal{M}$ over $\mathbb{Z}\left[\left(S_{i}\right)^{-1}\right]$ is isomorphic to $\mathcal{M}_{\mathbb{Z}\left[\left(S_{i}\right)^{-1}\right]}$. Moreover, the vector bundle $\boldsymbol{V}_{\mathrm{dR}, M}$ on $M$, along with its integrable connection and the isotropic line $\boldsymbol{V}_{\mathrm{dR}, M}^{1}$, has a canonical extension $\left(\boldsymbol{V}_{\mathrm{dR}}, \boldsymbol{V}_{\mathrm{dR}}^{1}\right)$ over $\mathcal{M}$.

Proof. This is immediate from Proposition 2.4.5.

Definition 2.4.7. The line bundle $\boldsymbol{V}_{\mathrm{dR}}^{1} \subset \boldsymbol{V}_{\mathrm{dR}}$ is the tautological bundle on $\mathcal{M}$. Its dual is the cotautological bundle.

Remark 2.4.8. Under the uniformization $\mathcal{D} \rightarrow \Gamma_{g} \backslash \mathcal{D} \subset \mathcal{M}(\mathbb{C})$, of (2.3), and using the notation of (2.1), the tautological bundle and cotautological bundle pull-back to the line bundles $z \mapsto \mathbb{C} z$ and $z \mapsto V_{\mathbb{C}} /(\mathbb{C} z)^{\perp}$, respectively.

By (7.12) of [MP16], the Kuga-Satake abelian scheme over $M$ extends (necessarily uniquely) to an abelian scheme

$$
\pi: \mathcal{A} \rightarrow \mathcal{M}
$$


endowed with a right $C(L)$-action and $\mathbb{Z} / 2 \mathbb{Z}$-grading. The de Rham realization of $\mathcal{A}$ provides us with an extension $\boldsymbol{H}_{\mathrm{dR}}$ over $\mathcal{M}$ of the filtered vector bundle with connection $\boldsymbol{H}_{\mathrm{dR}, M}$, equipped with its $\mathbb{Z} / 2 \mathbb{Z}$-grading and right $C(L)$-action. Let

$$
\boldsymbol{H}_{\text {crys }}=\underline{\operatorname{Hom}}\left(R^{1} \pi_{\text {crys }, *} \mathcal{O}_{\mathcal{A}_{\mathbb{F} p} / \mathbb{Z}_{p}}^{\text {crys }}, \mathcal{O}_{\mathcal{M}_{\mathbb{F}_{p}} / \mathbb{Z}_{p}}^{\text {crys }}\right)
$$

be the first crystalline homology of $\mathcal{A}_{\mathbb{F}_{p}}$ relative to $\mathcal{M}_{\mathbb{F}_{p}}$.

Denote by 1 the structure sheaf $\mathcal{O}_{\mathcal{M}}$ with its standard connection and trivial filtration in the de Rham case; the constant sheaf $\underline{\mathbb{Z}}_{p}$ on $\mathcal{M}[1 / p]$ in the étale case; the crystal $\mathcal{O}_{\mathcal{M}_{\mathbb{F}}, \text { crys }}$ in the crystalline case; and the trivial variation of $\mathbb{Z}$-Hodge structures over $M_{\mathbb{C}}^{\text {an }}$ in the Betti case. If $\boldsymbol{H}$ is any one of $\boldsymbol{H}_{\mathrm{dR}}, \boldsymbol{H}_{\ell, \text { et }}, \boldsymbol{H}_{\text {crys }}$, or $\boldsymbol{H}_{\mathrm{Be}}$, then $\boldsymbol{H}$ is a $\mathbf{1}$-module in the appropriate category.

It follows from [MP16, (3.12),(7.13)] that there is a canonical local direct summand

$$
\boldsymbol{V} \subset \underline{\operatorname{End}}_{C(L)}(\boldsymbol{H})
$$

of grade shifting endomorphisms. In fact, as we will see below in Proposition 2.5.1, the homological realizations of the canonical isometric embedding $\Lambda \hookrightarrow \operatorname{End}\left(\left.\mathcal{A}^{\diamond}\right|_{\mathcal{M}}\right)$ exhibit $\Lambda \otimes \mathbf{1}$ as an isometric local direct summand of $\left.\boldsymbol{V}^{\diamond}\right|_{\mathcal{M}}$, and

$$
\boldsymbol{V}=\left.(\Lambda \otimes \mathbf{1})^{\perp} \subset \boldsymbol{V}^{\diamond}\right|_{\mathcal{M}}
$$

In the de Rham or Betti case, $\boldsymbol{V}$ is identified with the realizations already constructed above. Moreover, for any prime $p$, the étale realization $\boldsymbol{V}_{p \text {,et }} \subset \underline{\operatorname{End}}_{C(L)}\left(\boldsymbol{H}_{p \text {,et }}\right)$ is the unique extension of that over $\mathcal{M}[1 / p]$. Here, we are using the following fact: Given a normal flat Noetherian algebraic $\mathbb{Z}[1 / p]$-stack $S$, and a lisse $p$-adic sheaf $F$ over $S$, any lisse subsheaf of $\left.F\right|_{S_{\mathbb{Q}}}$ extends uniquely to a lisse subsheaf of $F$. To see this, we can reduce to the case where $S$ is a normal, connected, flat Noetherian $\mathbb{Z}[1 / p]$-scheme, where the assertion amounts to saying that the map of étale fundamental groups $\pi_{1}\left(S_{\mathbb{Q}}\right) \rightarrow \pi_{1}(S)$ is surjective. This is shown in [Gro03, Exp. V, Prop. 8.2].

For any section $x$ of $\boldsymbol{V}$, the element $x \circ x$ is a scalar endomorphism of $\boldsymbol{H}$. Define a quadratic form

$$
Q: V \rightarrow 1
$$

by $x \circ x=\boldsymbol{Q}(x) \cdot \mathrm{Id}$.

Remark 2.4.9. Although we will not require this in what follows, we note that there is a certain compatibility between the crystalline realization $\boldsymbol{V}_{\text {crys }}$ over $\mathcal{M}_{\mathbb{F}_{p}}$ and the $p$-adic realization $\boldsymbol{V}_{p \text {,et }}$ over $\mathcal{M}[1 / p]$. Suppose that we are given a finite extension $F / \mathbb{Q}_{p}$, contained in an algebraic closure $\overline{\mathbb{Q}}_{p}$ of $\mathbb{Q}_{p}$, with residue field $k$. Write $F_{0} \subset F$ for the maximal unramified subextension of $F$. Suppose that we are given a point $s \in \mathcal{M}(F)$ specializing to a point $s_{0} \in \mathcal{M}(k)$. Write $\bar{s}$ for the corresponding $\overline{\mathbb{Q}}_{p}$-point of $\mathcal{M}$. Then the comparison isomorphism between the crystalline cohomology of $\mathcal{A}_{s_{0}}$ with respect to $W(k)=\mathcal{O}_{F_{0}}$ and the $p$-adic étale cohomology of $\mathcal{A}_{\bar{s}}$ induces, by $[\mathrm{MP} 16,(7.11)]$, an isometry

$$
\boldsymbol{V}_{\text {crys }, s_{0}} \otimes_{F_{0}} B_{\text {crys }} \stackrel{\simeq}{\rightarrow} V_{p, \text { et }, \overline{\mathbf{s}}} \otimes_{\mathbb{Q}_{p}} B_{\text {crys }}
$$

\subsection{Functoriality of integral models}

We consider the question of functoriality of the previous constructions with respect to the maximal quadratic space $L$. Let $L_{0} \subset L$ be an isometric embedding of maximal quadratic spaces over $\mathbb{Z}$, of signatures $\left(n_{0}, 2\right)$ and $(n, 2)$, respectively. This implies that $L_{0}$ is a $\mathbb{Z}$-module direct summand of $L$. We will maintain our assumption that $n_{0} \geqslant 1$. 


\section{Height PAIRINGS ON ORTHOGONAL SHIMURA VARIETIES}

The quadratic spaces $L_{0}$ and $L$ have associated Shimura varieties $M_{0}$ and $M$, with level structure defined by the compact open subgroups

$$
\begin{aligned}
K_{0} & =\operatorname{GSpin}\left(L_{0} \otimes \mathbb{A}_{f}\right) \cap C\left(\widehat{L}_{0}\right)^{\times} \\
K & =\operatorname{GSpin}\left(L \otimes \mathbb{A}_{f}\right) \cap C(\widehat{L})^{\times} .
\end{aligned}
$$

The inclusion $L_{0} \hookrightarrow L$ induces a homomorphism of Clifford algebras, which then restricts to an inclusion of algebraic groups $\operatorname{GSpin}\left(V_{0}\right) \rightarrow \operatorname{GSpin}(V)$ satisfying $K_{0}=K \cap \operatorname{GSpin}\left(V_{0}\right)\left(\mathbb{A}_{f}\right)$. By the theory of canonical models of Shimura varieties, there is an induced finite and unramified morphism of $\mathbb{Q}$-stacks $M_{0} \rightarrow M$.

Let $\mathcal{M}_{0}$ and $\mathcal{M}$ be the integral models over $\mathbb{Z}$, and let $\mathcal{A}_{0} \rightarrow \mathcal{M}_{0}$ and $\mathcal{A} \rightarrow \mathcal{M}$ be their Kuga-Satake abelian schemes. They are equipped with actions of $C\left(L_{0}\right)$ and $C(L)$, respectively. Let $\boldsymbol{H}_{0}$ and $\boldsymbol{H}$ stand for the various homological realizations of $\mathcal{A}_{0}$ and $\mathcal{A}$, respectively. We have the canonical sub-objects $\boldsymbol{V}_{0} \subset \underline{\text { End }}_{C\left(L_{0}\right)}\left(\boldsymbol{H}_{0}\right)$ and $\boldsymbol{V} \subset \underline{\text { End }}_{C(L)}(\boldsymbol{H})$ introduced in $\S 2.4$.

The Kuga-Satake abelian schemes over $M_{0}$ and $M$ are determined by the lattices $C\left(L_{0}\right) \subset$ $C\left(V_{0}\right)$ and $C(L) \subset C(V)$ (in the sense of [Del72, §4.12]), and the argument in [MP16, (6.4)] shows that there is a $C(L)$-linear isomorphism of abelian schemes

$$
\left.\left.\left(\mathcal{A}_{0} \otimes_{C\left(L_{0}\right)} C(L)\right)\right|_{M_{0}} \stackrel{\simeq}{\rightarrow} \mathcal{A}\right|_{M_{0}}
$$

over the generic fiber $M_{0}$ of $\mathcal{M}_{0}$. As $L_{0}$ is a direct summand of $L$, one easily checks that $C(L)$ is a free $C\left(L_{0}\right)$-module, and so the tensor construction $\mathcal{A}_{0} \otimes_{C\left(L_{0}\right)} C(L)$ in $(2.11)$ is defined.

Proposition 2.5.1. The morphism $M_{0} \rightarrow M$ extends uniquely to an unramified morphism $\mathcal{M}_{0} \rightarrow \mathcal{M}$, and (2.11) extends to a $C(L)$-linear isomorphism of abelian schemes

$$
\left.\mathcal{A}_{0} \otimes_{C\left(L_{0}\right)} C(L) \stackrel{\simeq}{\rightarrow} \mathcal{A}\right|_{\mathcal{M}_{0}}
$$

over $\mathcal{M}_{0}$. In particular,

$$
\left.\boldsymbol{H}_{0} \otimes_{C\left(L_{0}\right)} C(L) \stackrel{\simeq}{\rightarrow} \boldsymbol{H}\right|_{\mathcal{M}_{0}}
$$

Furthermore, if we set $\Lambda=\left\{\lambda \in L: \lambda \perp L_{0}\right\}$ and define a sheaf $\boldsymbol{\Lambda}=\Lambda \otimes \mathbf{1}$ on $\mathcal{M}_{0}$, then there is a canonical embedding $\Lambda \subset \operatorname{End}\left(\left.\mathcal{A}\right|_{\mathcal{M}_{0}}\right)$ with the following properties:

(i) Its homological realization $\boldsymbol{\Lambda} \hookrightarrow \underline{\operatorname{End}}\left(\left.\boldsymbol{H}\right|_{\mathcal{M}_{0}}\right)$ arises from an isometric map $\left.\boldsymbol{\Lambda} \hookrightarrow \boldsymbol{V}\right|_{\mathcal{M}_{0}}$, and exhibits $\boldsymbol{\Lambda}$ as a local direct summand of $\left.\boldsymbol{V}\right|_{\mathcal{M}_{0}}$.

(ii) The injection

$$
\underline{\operatorname{End}}_{C\left(L_{0}\right)}\left(\boldsymbol{H}_{0}\right) \rightarrow \underline{\operatorname{End}}_{C(L)}\left(\left.\boldsymbol{H}\right|_{\mathcal{M}_{0}}\right)
$$

induced by (2.13) identifies $\boldsymbol{V}_{0}$ with the submodule of all elements of $\left.\boldsymbol{V}\right|_{\mathcal{M}_{0}}$ anticommuting with all elements of $\boldsymbol{\Lambda}$. Furthermore, $\left.\boldsymbol{V}_{0} \subset \boldsymbol{V}\right|_{\mathcal{M}_{0}}$ is locally a direct summand.

(iii) In the de Rham case, the inclusion $\left.\boldsymbol{V}_{\mathrm{dR}} \subset \boldsymbol{V}_{\mathrm{dR}}\right|_{\mathcal{M}_{0}}$ identifies

$$
\left.\boldsymbol{V}_{0, \mathrm{dR}}^{1} \stackrel{\simeq}{\rightarrow} \boldsymbol{V}_{\mathrm{dR}}^{1}\right|_{\mathcal{M}_{0}} \text {. }
$$

Proof. It suffices to construct the morphism $\mathcal{M}_{0} \rightarrow \mathcal{M}$ after localizing at a prime $p$. So we can assume that we have a sequence of isometric embeddings $L_{0} \hookrightarrow L \hookrightarrow L^{\diamond}$, where $L^{\diamond}$ is maximal and self-dual at $p$.

By taking the normalizations of $\mathcal{M}_{\mathbb{Z}_{(p)}}^{\diamond}$ in $M_{0}$ and $M$, respectively, we see that the finite morphism $M_{0} \rightarrow M$ extends to a map

$$
\check{\mathcal{M}}_{0, \mathbb{Z}_{(p)}} \rightarrow \check{\mathcal{M}}_{\mathbb{Z}_{(p)}}
$$




\section{F. Andreatta, E. Z. Goren, B. Howard, K. Madapusi Pera}

Set $\Lambda_{1}=L^{\perp} \subset L^{\diamond}$ and $\widetilde{\Lambda}=L_{0}^{\perp} \subset L^{\diamond}$, so that $\Lambda_{1}$ is a direct summand in $\widetilde{\Lambda}$ with orthogonal complement $\Lambda$. From this, it is clear that (2.14) restricts to a map $\check{\mathcal{M}}_{0, \mathbb{Z}_{(p)}}^{\mathrm{pr}} \rightarrow \check{\mathcal{M}}_{\mathbb{Z}_{(p)}}^{\mathrm{pr}}$. We can deduce from Proposition 2.4.3 that, étale locally on the source, this map is isomorphic to an étale neighborhood of the map of quadrics $\mathrm{M}^{\mathrm{loc}}\left(L_{0}\right) \rightarrow \mathrm{M}^{\mathrm{loc}}(L)$. In particular, it is unramified.

To check that this carries $\mathcal{M}_{0, \mathbb{Z}_{(p)}}$ to $\mathcal{M}_{\mathbb{Z}_{(p)}}$ it suffices now to show that the map

$$
\mathrm{M}^{\mathrm{loc}}\left(L_{0}\right)_{\mathbb{Z}_{(p)}} \rightarrow \mathrm{M}^{\mathrm{loc}}(L)_{\mathbb{Z}_{(p)}}
$$

of quadrics over $\mathbb{Z}_{(p)}$ carries the smooth locus to the smooth locus and, if $p \neq 2$, the regular locus of the source into that of the target.

For the first assertion, observe that an $\overline{\mathbb{F}}_{p}$-valued point of the space is in the smooth locus if and only if it corresponds to an isotropic line that is not contained in the radical of $L_{0, \overline{\mathbb{F}}_{p}}$. But then it is a fortiori not in the radical of $L_{\overline{\mathbb{F}}_{p}}$, and hence corresponds to a smooth point of $\mathrm{M}^{\text {loc }}(L)$ as well.

We are left to prove the second claim, so that we can assume that $p$ is odd.

If the discriminant of $L_{\mathbb{Z}_{(p)}}$ is not divisible by $p^{2}$, the entire target is regular (see [MP16, (2.16)]), and there is nothing to check. Otherwise, the locus where $\mathrm{M}^{\text {loc }}(L)_{\mathbb{Z}_{(p)}}$ is not regular consists exactly of the two $\mathbb{F}_{p^{2}}$-valued points where the corresponding isotropic line $L^{1} \subset L_{\mathbb{F}_{p^{2}}}$ lies in the radical $\operatorname{rad}\left(L_{\mathbb{F}_{p^{2}}}\right)$ and is also isotropic for the non-degenerate quadratic form on this radical (see loc. cit.).

Suppose therefore that we have an isotropic line $L_{0, \bar{F}_{p}}^{1} \subset L_{0, \overline{\mathbb{F}}_{p}}$. We need to show: If, as an isotropic line in $L_{\overline{\mathbb{F}}_{p}}$, it corresponds to one of the two singular points above, then the discriminant of $L_{0}$ is also divisible by $p^{2}$, and $L_{0, \overline{\mathbb{F}}_{p}}^{1}$ corresponds to a singular point of $\mathrm{M}^{\mathrm{loc}}\left(L_{0}\right)$.

As any smooth point of $\mathrm{M}^{\mathrm{loc}}\left(L_{0}\right)$ (that is, a point at which the space is smooth over $\mathbb{Z}$ ) maps to a smooth point of $\mathrm{M}^{\mathrm{loc}}(L)$, the only remaining possibility is that the discriminant of $L_{0}$ is divisible exactly by $p$, and that $L_{0, \overline{\mathbb{F}}_{p}}^{1}$ is just the radical of $L_{0, \overline{\mathbb{F}}_{p}}$, corresponding to the unique nonsmooth point of $\mathrm{M}^{\mathrm{loc}}\left(L_{0}\right)_{\mathbb{Z}_{(p)}}$. However, this point is defined over $\mathbb{F}_{p}$, whereas both the singular points of $\mathrm{M}^{\mathrm{loc}}(L)_{\mathbb{Z}_{(p)}}$ are only defined over $\mathbb{F}_{p^{2}}$. So this possibility can also be excluded.

Now, the fact that the isomorphism of abelian schemes also extends over $\mathcal{M}_{0}$ is a consequence of [FC90, Proposition I.2.7].

The canonical embedding $\Lambda \subset \operatorname{End}\left(\left.\mathcal{A}\right|_{\mathcal{M}_{0, \mathbb{Z}_{(p)}}}\right)$ is constructed as follows: First, we will define an action of $\Lambda$ on

$$
\mathcal{A}_{0} \otimes_{\mathbb{Z}} C(L)=\left(\mathcal{A}_{0}^{+} \otimes_{\mathbb{Z}} C(L)\right) \times\left(\mathcal{A}_{0}^{-} \otimes_{\mathbb{Z}} C(L)\right) .
$$

Given a section of the form $\left(a^{+} \otimes c, a^{-} \otimes c^{\prime}\right)$ of this product and an element $\lambda \in \Lambda$, we set

$$
\lambda \cdot\left(a^{+} \otimes c, a^{-} \otimes c^{\prime}\right)=\left(a^{+} \otimes \lambda c,-a^{-} \otimes \lambda c^{\prime}\right) .
$$

As $L_{0}$ anti-commutes with $\Lambda$ within $C(L)$, it is easy to check that this action of $\Lambda$ descends to one on

$$
\left.\mathcal{A}_{0} \otimes_{C\left(L_{0}\right)} C(L) \stackrel{(2.12)}{\simeq} \mathcal{A}\right|_{\mathcal{M}_{0, \mathbb{Z}}(p)} .
$$

Claims (i), (ii) and (iii) are now shown in [MP16, (7.13)] for the crystalline realizations over $\mathcal{M}_{0, \mathbb{F}_{p}}$, but in the case where $L$ is self-dual at $p$. From this, we can easily deduce the general case, just as above, by embedding everything in a quadratic space that is self-dual at $p$. 


\subsection{Special endomorphisms}

For any scheme $S \rightarrow \mathcal{M}$ the pull-back $\mathcal{A}_{S}$ of the Kuga-Satake abelian scheme has a distinguished submodule

$$
V\left(\mathcal{A}_{S}\right) \subset \operatorname{End}_{C(L)}\left(\mathcal{A}_{S}\right)
$$

of special endomorphisms, defined in [MP16, (5.4)]. If $S$ is connected and $s \rightarrow S$ is any geometric point, there is a cartesian diagram

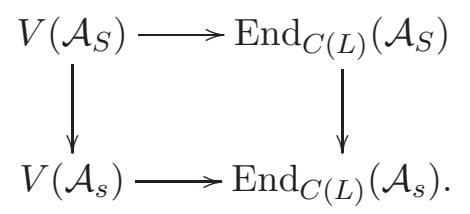

In other words, if $S$ is connected, an endomorphism is special if and only if it is special at one (equivalently, all) geometric points of $S$.

At a geometric point $s$, the property of being special can be characterized homologically, using the subspace

$$
\boldsymbol{V}_{s} \subset \operatorname{End}_{C(L)}\left(\boldsymbol{H}_{s}\right)
$$

If $s=\operatorname{Spec}(\mathbb{C})$ then $x \in \operatorname{End}_{C(L)}\left(\mathcal{A}_{s}\right)$ is special if and only if its Betti realization lies in the subspace (2.15). This is equivalent to the $\ell$-adic étale realization of $x$ lying in (2.15) for one (equivalently, all) primes $\ell$. It is also equivalent to requiring that the de Rham realization $t_{\mathrm{dR}}(x) \in$ $\operatorname{End}_{C(L)}\left(\boldsymbol{H}_{\mathrm{dR}, s}\right)$ lie in the subspace $\boldsymbol{V}_{\mathrm{dR}, s}$.

If $s$ is a geometric point of characteristic $p$, then $x \in \operatorname{End}_{C(L)}\left(\mathcal{A}_{s}\right)$ is special if and only if its crystalline realization lies in (2.15). This implies that the $\ell$-adic étale realization lies in (2.15) for all $\ell$ different from the characteristic of $s$; cf. [MP16, (5.22)].

Fix an element $\mu \in L^{\vee} / L$ and a prime $p>0$, and suppose that we are given an embedding $L \hookrightarrow L^{\diamond}$ of maximal quadratic lattices with $L^{\diamond}$ self-dual at $p$. Let $\Lambda=L^{\perp} \subset L^{\diamond}$ be the subspace of elements orthogonal to $L$.

There are canonical isomorphisms

$$
\mathbb{Z}_{p} \otimes\left(L^{\vee} / L\right) \stackrel{\simeq}{\leftarrow} \mathbb{Z}_{p} \otimes\left(L^{\diamond} /(L \oplus \Lambda)\right) \stackrel{\simeq}{\rightarrow} \mathbb{Z}_{p} \otimes\left(\Lambda^{\vee} / \Lambda\right),
$$

which allow us to view $\mu_{p}$ as an element of $\mathbb{Z}_{p} \otimes\left(\Lambda^{\vee} / \Lambda\right)$. Fix a lift $\tilde{\mu}_{p} \in \Lambda^{\vee}$ of $\mu_{p}$.

By construction, the map $\Lambda \hookrightarrow \operatorname{End}\left(\mathcal{A}_{S}^{\diamond}\right)$ from Proposition 2.5.1 factors through an isometric embedding $\Lambda \hookrightarrow V\left(\mathcal{A}_{S}^{\diamond}\right)$. Via this embedding, we can view $\tilde{\mu}_{p}$ as an element of $V\left(\mathcal{A}_{S}^{\diamond}\right)_{\mathbb{Q}}$.

LEMMA 2.6.1. The subset

$$
V_{\mu}\left(\mathcal{A}_{S}\right)_{(p)}=\left\{x \in V\left(\mathcal{A}_{S}\right)_{\mathbb{Q}}: x+\tilde{\mu}_{p} \in V\left(\mathcal{A}_{S}^{\diamond}\right) \otimes \mathbb{Z}_{(p)}\right\} \subset V\left(\mathcal{A}_{S}\right)_{\mathbb{Q}}
$$

is functorial in $S$, and is independent of the choice of embedding $L \hookrightarrow L^{\diamond}$ of maximal lattices with $L^{\diamond}$ self-dual at $p$.

Proof. Note that the definition is clearly independent of the choice of lift $\tilde{\mu}_{p}$.

The functoriality of the subset is clear, so it only remains to prove the independence from the choice of embedding into a self-dual at $p$ maximal lattice. For this, as in the proof of Proposition 2.4 .5 we can reduce to the case where both $L$ and $L^{\diamond}$ are self-dual at $p$, where we have to show that the definition using the identity $L \rightarrow L$ is equivalent to the one given using the embedding $L \hookrightarrow L^{\diamond}$. 


\section{F. Andreatta, E. Z. Goren, B. Howard, K. Madapusi Pera}

In this case, $\tilde{\mu}_{p}$ can be chosen to be 0 , and we are reduced to checking that an element $x \in V\left(\mathcal{A}_{S}\right)_{\mathbb{Q}}$ maps into $V\left(\mathcal{A}_{S}\right) \otimes \mathbb{Z}_{(p)}$ if and only if it maps into $V\left(\mathcal{A}_{S}^{\diamond}\right) \otimes \mathbb{Z}_{(p)}$. For this, it is enough to verify that the inclusion of abelian groups

$$
V\left(\mathcal{A}_{S}\right) \subset V\left(\mathcal{A}_{S}^{\diamond}\right)
$$

maps onto a $\mathbb{Z}$-direct summand. However, this follows from the fact that $\left.\boldsymbol{V} \subset \boldsymbol{V}^{\diamond}\right|_{\mathcal{M}}$ is a local direct summand; see Proposition 2.5.1(ii).

Remark 2.6.2. When $S$ is a scheme over $\mathcal{M}\left[p^{-1}\right]$, there is a more intrinsic description of the subset above. Note that the natural action of the compact open subgroup $K \subset G\left(\mathbb{A}_{f}\right)$ on $\widehat{L}^{\diamond}$ preserves $\widehat{L}$, and induces the trivial action on all the quotients involved in (2.16). Therefore, over $\mathcal{M}\left[p^{-1}\right]$, we have a canonical isometry

$$
\alpha_{p}: \underline{\mathbb{Z}}_{p} \otimes\left(L^{\vee} / L\right) \stackrel{\simeq}{\rightarrow} \boldsymbol{V}_{p}^{\vee} / \boldsymbol{V}_{p}
$$

of $p$-torsion étale sheaves. In particular, by taking the pre-image of $\alpha_{p}\left(1 \otimes \mu_{p}\right)$ in $\boldsymbol{V}_{p}^{\vee}$, we obtain a subsheaf of sets $\boldsymbol{V}_{\mu, p} \subset \boldsymbol{V}_{p}^{\vee}$. The subset $V_{\mu}\left(\mathcal{A}_{S}\right)_{(p)}$ consists precisely of those elements of $V\left(\mathcal{A}_{S}\right)_{\mathbb{Q}}$, whose $p$-adic realizations land in $\boldsymbol{V}_{\mu, p}$.

For a scheme $S \rightarrow \mathcal{M}$, we now define a natural subset $V_{\mu}\left(\mathcal{A}_{S}\right) \subset V\left(\mathcal{A}_{S}\right)_{\mathbb{Q}}$ by setting

$$
V_{\mu}\left(\mathcal{A}_{S}\right)=\bigcap_{p} V_{\mu}\left(\mathcal{A}_{S}\right)_{(p)} \cdot
$$

We record some properties of these spaces.

Proposition 2.6.3.

(i) If $\mu=0$, then $V_{0}\left(\mathcal{A}_{S}\right)=V\left(\mathcal{A}_{S}\right)$.

(ii) Each $x \in V_{\mu}\left(\mathcal{A}_{S}\right)$, viewed as an element of $\operatorname{End}\left(\mathcal{A}_{S}\right)_{\mathbb{Q}}$, shifts the grading on $\mathcal{A}_{S}$ and commutes with the right action of $C(L)$.

(iii) Moreover, $x \circ x=Q(x) \cdot \operatorname{Id}$ for some non-negative $Q(x) \in \mathbb{Q}$ satisfying

$$
Q(x) \equiv Q(\mu) \quad(\bmod \mathbb{Z}),
$$

and $Q(x)=0$ if and only if $x=0$.

Proof. The first statement was shown in the course of the proof of Lemma 2.6.1, where we found that $V_{\mu}\left(\mathcal{A}_{S}\right)$ is a subset of $V\left(\mathcal{A}_{S}\right) \otimes \mathbb{Z}_{(p)}$ whenever $\mu_{p}=0$.

The second follows from the construction of the space $V\left(\mathcal{A}_{S}\right)$, and the fact that the endomorphisms $\boldsymbol{V} \subset \underline{\operatorname{End}}(\boldsymbol{H})$ of (2.15) are grade shifting and commute with $C(L)$.

For the third, we first check that the quadratic form $Q(x)$ is positive definite. But this follows from the existence of a polarization on $\mathcal{A}_{S}$ such that every element of $V\left(\mathcal{A}_{S}\right) \otimes \mathbb{Q}$ is fixed under the corresponding Rosati involution; see the proof of [MP16, (5.12)]. We also have to show that, for each prime $p$, we have

$$
Q(x) \equiv Q(\mu) \quad\left(\bmod \mathbb{Z}_{(p)}\right)
$$

We can assume that we have chosen an embedding $L \hookrightarrow L^{\diamond}$ with $L^{\diamond}$ self-dual at $p$. In this case, with $\Lambda$ and $\tilde{\mu}_{p}$ as in Lemma 2.6.1, we find that, in terms of the quadratic form $Q^{\diamond}$ on $V\left(\mathcal{A}_{S}^{\diamond}\right)$, we have

$$
Q^{\diamond}\left(x+\tilde{\mu}_{p}\right) \equiv 0 \quad\left(\bmod \mathbb{Z}_{(p)}\right)
$$




\section{Height PAIRINGs on ORThogonal Shimura varieties}

It can be checked using Proposition 2.5.1(ii) that $V\left(\mathcal{A}_{S}\right)$ is orthogonal to $\tilde{\mu}_{p}$ as a subspace of $V\left(\mathcal{A}_{S}^{\diamond}\right)_{\mathbb{Q}}$. Therefore, we have

$$
Q(x)=Q^{\diamond}(x) \equiv-Q^{\diamond}\left(\tilde{\mu}_{p}\right) \quad\left(\bmod \mathbb{Z}_{(p)}\right) .
$$

On the other hand, recall that $\tilde{\mu}_{p} \subset \Lambda^{\vee}$ was defined to be a lift of the image of $\mu_{p}$ under the canonical isomorphism

$$
\left(L^{\vee} / L\right) \otimes \mathbb{Z}_{(p)} \stackrel{\simeq}{\rightarrow} \Lambda^{\vee} / \Lambda \otimes \mathbb{Z}_{(p)}
$$

associated with the embedding $L \hookrightarrow L^{\diamond}$. In other words, if $\mu_{p}^{\prime} \in L^{\vee}$ is any lift of $\mu_{p}$, we have $\mu_{p}^{\prime}+\tilde{\mu}_{p} \in L^{\diamond}$. So arguing just as in the previous paragraph, we find

$$
Q\left(\mu_{p}^{\prime}\right) \equiv-Q^{\diamond}\left(\tilde{\mu}_{p}\right) \quad\left(\bmod \mathbb{Z}_{(p)}\right) .
$$

Comparing (2.18) and (2.19) finishes the proof.

As before, suppose that we are given an embedding of maximal quadratic spaces $L_{0} \hookrightarrow L$ with $\Lambda=L_{0}^{\perp} \subset L$. In particular the inclusion $L^{\vee} \hookrightarrow L_{0}^{\vee} \oplus \Lambda^{\vee}$ induces an injection

$$
L^{\vee} /\left(L_{0} \oplus \Lambda\right) \hookrightarrow\left(L_{0}^{\vee} / L_{0}\right) \oplus\left(\Lambda^{\vee} / \Lambda\right) .
$$

Fix an $\mathcal{M}_{0}$-scheme $S \rightarrow \mathcal{M}_{0}$. Then, by construction, the map $\Lambda \hookrightarrow \operatorname{End}\left(\mathcal{A}_{S}\right)$ from Proposition 2.5.1 factors through an isometric embedding $\Lambda \hookrightarrow V\left(\mathcal{A}_{S}\right)$.

Proposition 2.6.4. Fix an $\mathcal{M}_{0}$-scheme $S \rightarrow \mathcal{M}_{0}$.

(i) There is a canonical isometry

$$
V\left(\mathcal{A}_{0, S}\right) \stackrel{\simeq}{\rightarrow} \Lambda^{\perp} \subset V\left(\mathcal{A}_{S}\right),
$$

where here $\Lambda^{\perp}$ is calculated inside $V\left(\mathcal{A}_{S}\right)$.

(ii) For every $\mu \in L^{\vee} / L$ and every $\left(\mu_{1}, \mu_{2}\right) \in(\mu+L) /\left(L_{0} \oplus \Lambda\right)$ the map (2.20), tensored with $\mathbb{Q}$, restricts to an injection

$$
V_{\mu_{1}}\left(\mathcal{A}_{0, S}\right) \times\left(\mu_{2}+\Lambda\right) \hookrightarrow V_{\mu}\left(\mathcal{A}_{S}\right) .
$$

(iii) The above injections determine a decomposition

$$
V_{\mu}\left(\mathcal{A}_{S}\right)=\bigsqcup_{\left(\mu_{1}, \mu_{2}\right) \in(\mu+L) /\left(L_{0} \oplus \Lambda\right)} V_{\mu_{1}}\left(\mathcal{A}_{0, S}\right) \times\left(\mu_{2}+\Lambda\right) .
$$

Proof. Claim (i) follows from the definitions and Proposition 2.5.1; see also [MP16, (7.15)]. In particular, any element $x \in V\left(\mathcal{A}_{S}\right) \otimes \mathbb{Q}$ admits a decomposition

$$
x=\left(x_{0}, \nu\right) \in\left(V\left(\mathcal{A}_{0, S}\right) \otimes \mathbb{Q}\right) \times(\Lambda \otimes \mathbb{Q}) .
$$

Using this decomposition, it is an easy exercise to deduce claims (ii) and (iii) from the definitions. The main input is Lemma 2.6.1, which permits us to study all spaces in question by embedding $L$ into a maximal quadratic lattice that is self-dual at any given prime $p$.

\subsection{Special divisors}

For $m \in \mathbb{Q}_{>0}$ and $\mu \in L^{\vee} / L$, define the special cycle $\mathcal{Z}(m, \mu) \rightarrow \mathcal{M}$ as the stack over $\mathcal{M}$ with functor of points

$$
\mathcal{Z}(m, \mu)(S)=\left\{x \in V_{\mu}\left(\mathcal{A}_{S}\right): Q(x)=m\right\}
$$

for any scheme $S \rightarrow \mathcal{M}$. Note that, by (2.17), the stack (2.21) is empty unless the image of $m$ in $\mathbb{Q} / \mathbb{Z}$ agrees with $Q(\mu)$. 
For later purposes we also define the stacks $\mathcal{Z}(0, \mu)$ in exactly the same way. As the only special endomorphism $x$ with $Q(x)=0$ is the zero map, we have

$$
\mathcal{Z}(0, \mu)= \begin{cases}\emptyset & \text { if } \mu \neq 0 \\ \mathcal{M} & \text { if } \mu=0\end{cases}
$$

The special cycles behave nicely under pullback by the morphism of Proposition 2.5.1. Indeed, the following is an immediate consequence of Proposition 2.6.4.

Proposition 2.7.1. Suppose that we are given an embedding of maximal quadratic spaces $L_{0} \hookrightarrow L$, and let $\Lambda \subset L$ be the submodule of vectors orthogonal to $L_{0}$. Let $\mathcal{M}_{0} \rightarrow \mathcal{M}$ be the corresponding morphism of Shimura varieties, as in Proposition 2.5.1. Thus, for any $m \in \mathbb{Q} \geqslant 0$ and $\mu \in L^{\vee} / L$ there is a special cycle $\mathcal{Z}(m, \mu) \rightarrow \mathcal{M}$, and for any $m_{1} \in \mathbb{Q} \geqslant 0$ and $\mu_{1} \in L_{0}^{\vee} / L_{0}$ there is a special cycle $\mathcal{Z}_{0}\left(m_{1}, \mu_{1}\right) \rightarrow \mathcal{M}_{0}$.

Then, there is an isomorphism of $\mathcal{M}_{0}$-stacks

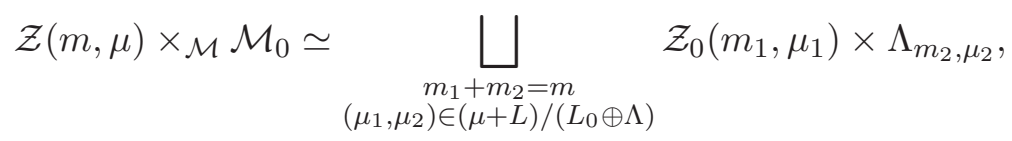

where

$$
\Lambda_{m_{2}, \mu_{2}}=\left\{x \in \mu_{2}+\Lambda: Q(x)=m_{2}\right\},
$$

and $\mathcal{Z}_{0}\left(m_{1}, \mu_{1}\right) \times \Lambda_{m_{2}, \mu_{2}}$ denotes the disjoint union of $\# \Lambda_{m_{2}, \mu_{2}}$ copies of $\mathcal{Z}_{0}\left(m_{1}, \mu_{1}\right)$.

Proposition 2.7.2. For every $m \in \mathbb{Q} \geqslant 0$ and $\mu \in L^{\vee} / L$, the morphism $\mathcal{Z}(m, \mu) \rightarrow \mathcal{M}$ is relatively representable, finite, and unramified.

Proof. Suppose that we have an embedding $L \hookrightarrow L^{\diamond}$ of maximal quadratic spaces with $\Lambda \subset L^{\diamond}$ the submodule of vectors orthogonal to $L$. Suppose also that $m^{\diamond} \in \mathbb{Q}_{\geqslant 0}, \mu^{\diamond} \in L^{\diamond, \vee} / L^{\diamond}$, and $\mu^{\prime} \in \Lambda^{\vee} / \Lambda$ are such that

$$
\left(\mu, \mu^{\prime}\right) \in\left(\mu^{\diamond}+L^{\diamond}\right) /(L \oplus \Lambda)
$$

and

$$
\begin{aligned}
& Q\left(\mu^{\diamond}\right) \equiv m^{\diamond} \quad(\bmod \mathbb{Z}) \\
& Q\left(\mu^{\prime}\right) \equiv m^{\diamond}-m \quad(\bmod \mathbb{Z}) .
\end{aligned}
$$

Let $\mathcal{Z}\left(m^{\diamond}, \mu^{\diamond}\right) \rightarrow \mathcal{M}^{\diamond}$ be the morphism of stacks associated with the triple $\left(L^{\diamond}, m^{\diamond}, \mu^{\diamond}\right)$. Suppose that we know that $\mathcal{Z}\left(m^{\diamond}, \mu^{\diamond}\right) \rightarrow \mathcal{M}^{\diamond}$ is relatively representable, finite, and unramified. Then Proposition 2.7.1 implies that $\mathcal{Z}(m, \mu)$ can be viewed as a closed and open substack of $\mathcal{Z}\left(m^{\diamond}, \mu^{\diamond}\right) \times \mathcal{M}_{\diamond} \mathcal{M}$, and is thus finite and unramified over $\mathcal{M}$.

Therefore, by replacing $L$ with an appropriate choice of $L^{\diamond}$, one reduces the problem to studying the morphism $\mathcal{Z}(m, \mu) \rightarrow \mathcal{M}$ under the additional assumption that $L$ is self-dual. Of course this implies that $\mu=0$.

Suppose we have a Noetherian scheme $S$ and a morphism $S \rightarrow \mathcal{M}$. Recall that the étale sheaf $\underline{\operatorname{End}}\left(\mathcal{A}_{S}\right)$ on $S$ is represented by a formally unramified scheme over $S$, whose connected components are projective. Formal unramifiedness follows from the rigidity of morphisms of abelian schemes [MFK94, Corollary 6.2]. Representability and projectiveness of the components follows from the theory of Hilbert schemes and the valuative criterion of properness, as in [Hida04, $\S 6.1 .5, \S 6.1 .6]$. 


\section{HeIgHT PAIRINGS ON ORTHOGONAL SHIMURA VARIETIES}

As in [MP16, Prop. 6.13], $\mathcal{Z}(m, \mu)_{S}$ is represented by a formally unramified $S$-scheme whose connected components are projective. More precisely, it is isomorphic to a union of connected components of $\underline{\operatorname{End}}\left(\mathcal{A}_{S}\right)$. This is a consequence of the fact that, for any $f \in \operatorname{End}\left(\mathcal{A}_{S}\right)$, the property of being special can be checked at any geometric point of a connected component of $S$, and similarly for the property $f \circ f=m$.

The only thing left to prove is that $\mathcal{Z}(m, \mu)_{S}$ is of finite type over $S$. For this, one first notes that, for any projective scheme $X$ over $S$, the Hilbert scheme parameterizing closed subschemes of $X$ with fixed Hilbert polynomial is projective, and hence of finite type, over $S$. See [FGA05, §5.6]. We will realize $\mathcal{Z}(m, \mu)_{S}$ as a closed subscheme of such a Hilbert scheme with $X=\mathcal{A}_{S} \times_{S} \mathcal{A}_{S}$.

Of course, the Hilbert polynomial depends on the choice of an ample line bundle on $X$. We will choose our line bundle as follows: Let $\mathcal{A}_{S}^{\vee}$ be the dual abelian scheme, and let $\mathcal{P}$ be the Poincaré line bundle on $\mathcal{A}_{S} \times{ }_{S} \mathcal{A}_{S}^{\vee}$. As in Proposition 2.6.3, one can choose a polarization $\psi: \mathcal{A}_{S} \rightarrow \mathcal{A}_{S}^{\vee}$ such that the associated Rosati involution fixes all special endomorphisms of $\mathcal{A}_{S}$. Define an ample line bundle on $\mathcal{A}_{S}$ by

$$
\mathcal{O}(1)=(1 \times \psi)^{*} \mathcal{P} .
$$

As usual, for any $n \in \mathbb{Z}$, set $\mathcal{O}(n)=\mathcal{O}(1)^{\otimes n}$. Define an ample line bundle on $X$ by $p_{1}^{*} \mathcal{O}(1) \otimes$ $p_{2}^{*} \mathcal{O}(1)$, where $p_{i}: X \rightarrow \mathcal{A}_{S}$ are the two projections.

Suppose that $s \rightarrow \mathcal{Z}(m, \mu)_{S}$ is a geometric point. It determines a geometric point $s \rightarrow S$, along with a special endomorphism $x \in V\left(\mathcal{A}_{s}\right)$ with $Q(x)=m$. The Hilbert polynomial of the graph $\Gamma_{x} \subset X_{s}$ is associated with the function

$$
n \mapsto h^{0}\left(\mathcal{A}_{s}, \mathcal{O}(n) \otimes x^{*} \mathcal{O}(n)\right) .
$$

Since $x$ is fixed by the Rosati involution, one easily checks that $x^{*} \mathcal{O}(n)=\mathcal{O}(m n)$. Therefore the Hilbert polynomial in question is the one attached to

$$
n \mapsto h^{0}\left(\mathcal{A}_{s}, \mathcal{O}((m+1) n)\right),
$$

which clearly depends only on $m$.

Sending a point of $\mathcal{Z}(m, \mu)_{S}$ to the graph of the corresponding special endomorphism defines a closed immersion of $\mathcal{Z}(m, \mu)_{S}$ into the Hilbert scheme of closed subschemes of $X$ with fixed Hilbert polynomial. This proves that $\mathcal{Z}(m, \mu)_{S}$ is of finite type over $S$, and completes the proof.

Lemma 2.7.3. For any positive $m$ and any $\mu$, the complex orbifold $\mathcal{Z}(m, \mu)(\mathbb{C})$ just defined agrees with (2.4).

Proof. Consider the uniformization $M(\mathbb{C})=G(\mathbb{Q}) \backslash \mathcal{D} \times G\left(\mathbb{A}_{f}\right) / K$. By construction, for every

$$
z=(\boldsymbol{h}, g) \in \mathcal{D} \times G\left(\mathbb{A}_{f}\right),
$$

the fiber of $\boldsymbol{V}_{\mathrm{Be}, \mathbb{Q}}$ at $z$ is the $\mathbb{Q}$-vector space $V$ with Hodge structure determined by the negative plane $\boldsymbol{h} \subset V_{\mathbb{R}}$. The $(0,0)$ part of $\boldsymbol{V}_{\mathrm{Be}, \mathbb{Q}, z}$ is $V \cap \boldsymbol{h}^{\perp}$. The fibers of the subsheaves $\boldsymbol{V}_{\mathrm{Be}}$ and $\boldsymbol{V}_{\mathrm{Be}}^{\vee}$ of $\boldsymbol{V}_{\mathrm{Be}, \mathbb{Q}}$ at $z$ are $\widehat{L}_{g} \cap V=L_{g}$ and $\widehat{L}_{g}^{\vee} \cap V=L_{g}^{\vee}$, respectively. Here, using the notation of $\S 2.2$, $\widehat{L}_{g}=g \bullet \widehat{L}$ and $\widehat{L}_{g}^{\vee}=g \bullet \widehat{L}^{\vee}$.

By definition, we now have

$$
V\left(\mathcal{A}_{z}\right)=\operatorname{End}\left(\mathcal{A}_{z}\right) \cap \boldsymbol{V}_{\mathrm{Be}, \mathrm{z}}=\left(\boldsymbol{V}_{\mathrm{Be}, z}\right)^{(0,0)}=L_{g} \cap \boldsymbol{h}^{\perp} .
$$

This proves the lemma for the trivial class $\mu$. 


\section{F. Andreatta, E. Z. Goren, B. Howard, K. Madapusi Pera}

Now, since $K$ acts trivially on $L^{\vee} / L$, there is a canonical isometry $\boldsymbol{V}_{\mathrm{Be}}^{\vee} / \boldsymbol{V}_{\mathrm{Be}} \simeq L^{\vee} / L \otimes \mathbf{1}$ of torsion sheaves on $M(\mathbb{C})$. This determines, for every $\mu \in L^{\vee} / L$, a locally constant intermediate sheaf of sets $\boldsymbol{V}_{\mu, \mathrm{Be}} \subset \boldsymbol{V}_{\mathrm{Be}}^{\vee}$ whose fiber $\boldsymbol{V}_{\mu, \mathrm{Be}, z}$ is $\mu_{g}+L_{g}$. It is not hard to deduce from Remark 2.6.2 that $V_{\mu}\left(\mathcal{A}_{z}\right)$ consists precisely of those elements of $V\left(\mathcal{A}_{z}\right)_{\mathbb{Q}}$ whose Betti realization lands in $\boldsymbol{V}_{\mu, \mathrm{Be}}$. It follows that

$$
V_{\mu}\left(\mathcal{A}_{z}\right)=\left(\mu_{g}+L_{g}\right) \cap \boldsymbol{h}^{\perp},
$$

which proves the lemma for non-trivial $\mu$.

We now show that the stacks $\mathcal{Z}(m, \mu)$ with $m>0$ define (étale local) Cartier divisors on $\mathcal{M}$. Proposition 2.7.4. Suppose $m>0$. Then étale locally on the source, $\mathcal{Z}(m, \mu)$ defines a Cartier divisor on $\mathcal{M}$. More precisely: around every geometric point of $\mathcal{M}$ there is an étale neighborhood $U$ such that for every connected component $Z \subset \mathcal{Z}(m, \mu)_{U}$, the map $Z \rightarrow U$ is a closed immersion defined by a single non-zero equation.

Proof. Suppose that we are given a geometric point $z$ of $\mathcal{Z}(m, \mu)$, corresponding to an element $x \in V_{\mu}\left(\mathcal{A}_{z}\right)$. Let $R=\mathcal{O}_{\mathcal{M}, z}$ be the étale local ring of $\mathcal{M}$ at $z$. Since $\mathcal{Z}(m, \mu) \rightarrow \mathcal{M}$ is unramified, it follows that

$$
\mathcal{O}_{\mathcal{Z}(m, \mu), z} \stackrel{\simeq}{\rightarrow} R / J_{x}
$$

for some ideal $J_{x} \subset R$. Set $S=\operatorname{Spec}\left(R / J_{x}\right)$, so that $x \in V_{\mu}\left(\mathcal{A}_{z}\right)$ has a universal deformation to $x_{S} \in V_{\mu}\left(\mathcal{A}_{S}\right)$.

Fix an embedding $L \hookrightarrow L^{\diamond}$ so that $L^{\diamond}$ is maximal and self-dual at $p$ of signature $\left(n^{\diamond}, 2\right)$, and let $\Lambda \subset L^{\diamond}$ be the subset of vectors orthogonal to $L$. Let $\mathcal{A}^{\diamond} \rightarrow \mathcal{M}^{\diamond}$ be the Kuga-Satake abelian scheme over the integral model of the Shimura variety determined by $L^{\diamond}$. Proposition 2.6.4 (with $\mathcal{A}_{0}$ and $\mathcal{A}$ replaced by $\mathcal{A}$ and $\mathcal{A}^{\diamond}$ ) gives an inclusion

$$
V\left(\mathcal{A}_{S}\right) \oplus \Lambda \hookrightarrow V\left(\mathcal{A}_{S}^{\diamond}\right),
$$

which becomes an isomorphism after tensoring with $\mathbb{Q}$. There is a $\nu \in \Lambda^{\vee}$ such that the vector

$$
x_{S}^{\diamond}:=x_{S}+\nu \in\left(V\left(\mathcal{A}_{S}\right) \otimes \mathbb{Q}\right) \oplus(\Lambda \otimes \mathbb{Q})
$$

lies in the $\mathbb{Z}$-lattice $V\left(\mathcal{A}_{S}^{\diamond}\right)$. The crystalline realization $t_{\text {crys }}\left(x^{\diamond}\right)$ allows us to canonically lift the de Rham realization

to an element

$$
t_{\mathrm{dR}}\left(x_{S}^{\diamond}\right) \in \boldsymbol{V}_{\mathrm{dR}, R / J_{x}}^{\diamond}
$$

$$
t_{\text {crys }}\left(x_{S}^{\diamond}\right) \in \boldsymbol{V}_{\mathrm{dR}, R / J_{x}^{2}}^{\diamond}
$$

through the divided power thickening $S \hookrightarrow \operatorname{Spec}\left(R / J_{x}^{2}\right)$.

It follows from [MP16, (5.15)] that $t_{\mathrm{dR}}\left(x_{S}^{\diamond}\right)$ is orthogonal to $\boldsymbol{V}_{\mathrm{dR}, S}^{\diamond}$. Thus, if

$$
\omega \in \boldsymbol{V}_{\mathrm{dR}, R / J_{x}^{2}}^{1} \stackrel{\simeq}{\rightarrow} \boldsymbol{V}_{\mathrm{dR}, R / J_{x}^{2}}^{\diamond}
$$

is an $R$-module generator, we obtain an element $\left[\omega, t_{\text {crys }}\left(x_{S}^{\diamond}\right)\right] \in J_{x} / J_{x}^{2}$ using the pairing on $\boldsymbol{V}_{\mathrm{dR}}^{\diamond}$. In fact, Grothendieck-Messing theory $[\mathrm{MP} 16,(5.17)]$ shows that $\left[\omega, t_{\text {crys }}\left(x_{S}^{\diamond}\right)\right]$ generates $J_{x} / J_{x}^{2}$. The element

$$
t_{\text {crys }}\left(x_{S}\right):=t_{\text {crys }}\left(x_{S}^{\diamond}\right)-\nu
$$

lies in $\boldsymbol{V}_{\mathrm{dR}, R / J_{x}^{2}}^{\mathrm{V}}$, and the orthogonality relation $[\omega, \nu]=0$ implies

$$
\left[\omega, t_{\text {crys }}\left(x_{S}\right)\right]=\left[\omega, t_{\text {crys }}\left(x_{S}^{\diamond}\right)\right] .
$$


Lemma 2.7.5. The element $\left[\omega, t_{\text {crys }}\left(x_{S}\right)\right]$ generates $J_{x} / J_{x}^{2}$ as an $R$-module. In particular, $J_{x} \subset R$ is a principal ideal.

Proof. The discussion above proves the first assertion. The second follows by Nakayama's lemma.

Proposition 2.7.4 follows immediately from the lemma.

Remark 2.7.6. It follows from Proposition 2.7.4 that $\mathcal{Z}(m, \mu)$ is of pure $\operatorname{dimension} \operatorname{dim}(\mathcal{M})-1$. The morphism to $\mathcal{M}$ is not itself a closed immersion, but nevertheless $\mathcal{Z}(m, \mu)$ defines a Cartier divisor on $\mathcal{M}$, as in $[\mathrm{BHY} 15, \S 3.1]$.

\section{Harmonic modular forms and special divisors}

Fix a maximal quadratic space $L$ over $\mathbb{Z}$ of signature $(n, 2)$ with $n \geqslant 1$, set $V=L_{\mathbb{Q}}$, and let $\mathcal{M} \rightarrow \operatorname{Spec}(\mathbb{Z})$ be the corresponding integral model of the Shimura variety (2.3) defined in $\S 2$.

This section is a rapid review of some results and constructions of Bruinier [Bru02], BruinierFunke [BF04], and Bruinier-Yang [BY09]. In particular, we recall the construction of a divisor $\mathcal{Z}(f)$ and a Green function $\Phi(f)$ on $\mathcal{M}$ from a harmonic weak Maass form $f$.

\subsection{Vector valued modular forms}

Let $\mathfrak{S}_{L}$ be the (finite dimensional) space of $\mathbb{C}$-valued functions on $L^{\vee} / L$. As in [BY09] there is a Weil representation

$$
\omega_{L}:{\widetilde{\mathrm{SL}_{2}}}_{2}(\mathbb{Z}) \rightarrow \operatorname{Aut}_{\mathbb{C}}\left(\mathfrak{S}_{L}\right)
$$

where $\widetilde{\mathrm{SL}}_{2}(\mathbb{Z})$ is the metaplectic double cover of $\mathrm{SL}_{2}(\mathbb{Z})$. Define the conjugate action $\bar{\omega}_{L}$ by $\bar{\omega}_{L}(\gamma) \varphi=\overline{\omega_{L}(\gamma) \bar{\varphi}}$. We denote by $\omega_{L}^{\vee}$ the contragredient action of $\widetilde{\mathrm{SL}}_{2}(\mathbb{Z})$ on the complex linear dual $\mathfrak{S}_{L}^{\vee}$.

Remark 3.1.1. Our $\bar{\omega}_{L}$ is the representation denoted $\rho_{L}$ in [Bor98], [Bru02], [BF04], and [BY09]. If we denote by $-L$ the quadratic space over $\mathbb{Z}$ whose underlying $\mathbb{Z}$-module is $L$, but endowed with the quadratic form $-Q$, then $\mathfrak{S}_{L}=\mathfrak{S}_{-L}$ as vector spaces, but $\omega_{L}=\rho_{-L}$.

For a half-integer $k$, denote by $H_{k}\left(\omega_{L}\right)$ the $\mathbb{C}$-vector space of $\mathfrak{S}_{L}$-valued harmonic weak Maass forms of weight $k$ and representation $\omega_{L}$, in the sense ${ }^{2}$ of [BY09, §3.1]. As in [BY09, §3] there are subspaces

$$
S_{k}\left(\omega_{L}\right) \subset M_{k}^{!}\left(\omega_{L}\right) \subset H_{k}\left(\omega_{L}\right)
$$

of cusp forms and weakly modular forms. Define similar spaces of modular forms for the representation $\bar{\omega}_{L}$. Bruinier and Funke [BF04] have defined a differential operator

$$
\xi: H_{k}\left(\omega_{L}\right) \rightarrow S_{2-k}\left(\bar{\omega}_{L}\right)
$$

by $\xi(f)=2 i v^{k} \overline{(\partial f / \partial \bar{\tau})}$, where $\tau=u+i v$ is the variable on the upper half-plane, and have proved the exactness of the sequence

$$
0 \rightarrow M_{k}^{!}\left(\omega_{L}\right) \rightarrow H_{k}\left(\omega_{L}\right) \stackrel{\xi}{\rightarrow} S_{2-k}\left(\bar{\omega}_{L}\right) \rightarrow 0 .
$$

\footnotetext{
${ }^{2}$ These satisfy a more restrictive growth condition at the cusp than the weak Maass forms of [BF04, §3].
} 


\section{F. Andreatta, E. Z. Goren, B. Howard, K. Madapusi Pera}

Every $f(\tau) \in H_{k}\left(\omega_{L}\right)$ has an associated formal $q$-expansion

$$
f^{+}(\tau)=\sum_{\substack{m \in \mathbb{Q} \\-\infty \ll m}} c_{f}^{+}(m) \cdot q^{m},
$$

called the holomorphic part of $f$, with $c_{f}^{+}(m) \in \mathfrak{S}_{L}$. The delta functions $\varphi_{\mu}$ at elements $\mu \in L^{\vee} / L$ form a basis for $\mathfrak{S}_{L}$, and so each coefficient $c_{f}^{+}(m)$ can be decomposed as a sum

$$
c_{f}^{+}(m)=\sum_{\mu \in L^{\vee} / L} c_{f}^{+}(m, \mu) \cdot \varphi_{\mu}
$$

with $c_{f}^{+}(m, \mu) \in \mathbb{C}$. The transformation laws satisfied by $f$ imply that

$$
c_{f}^{+}(m, \mu)=c_{f}^{+}(m,-\mu),
$$

and also that $c_{f}^{+}(m, \mu)=0$, unless the image of $m$ in $\mathbb{Q} / \mathbb{Z}$ is equal to $-Q(\mu)$.

\subsection{Divisors and Green functions}

Suppose we are given a harmonic weak Maass form

$$
f(\tau) \in H_{1-\frac{n}{2}}\left(\omega_{L}\right)
$$

having integral principal part, in the sense that $c_{f}^{+}(m, \mu) \in \mathbb{Z}$ for all $\mu \in L^{\vee} / L$ and all $m<0$. Define a divisor on $\mathcal{M}$ by

$$
\mathcal{Z}(f)=\sum_{m \in \mathbb{Q}>0} \sum_{\mu \in L^{\vee} / L} c_{f}^{+}(-m, \mu) \mathcal{Z}(m, \mu) .
$$

Bruinier [Bru02], following ideas of Borcherds [Bor98] and Harvey-Moore, constructed a Green function $\Phi(f)$ for $\mathcal{Z}(f)$ as follows (see also [BY09, §4]). Let $G=\operatorname{GSpin}(V)$ as in $\S 2$. For each coset $g \in G\left(\mathbb{A}_{f}\right) / K$ there is a Siegel theta function

$$
\theta_{L}(\tau, z, g): \mathcal{H} \times \mathcal{D} \rightarrow \mathfrak{S}_{L}^{\vee}
$$

as in [BY09, (2.4)], which is $\Gamma_{g}$-invariant in the variable $z \in \mathcal{D}$, and transforms in the variable $\tau=u+i v \in \mathcal{H}$ like a modular form of representation $\omega_{L}^{\vee}$.

The regularized theta integral

$$
\Phi(f, z, g)=\int_{\mathcal{F}}^{\mathrm{reg}}\left\{f(\tau), \theta_{L}(\tau, z, g)\right\} \frac{d u d v}{v^{2}}
$$

of $\left[\right.$ BY09, (4.7)] defines a $\Gamma_{g}$-invariant function on $\mathcal{D}$, where

$$
\{\cdot, \cdot\}: \mathfrak{S}_{L} \times \mathfrak{S}_{L}^{\vee} \rightarrow \mathbb{C}
$$

is the tautological pairing. Letting $g$ vary and using the uniformization (2.3) yields a function $\Phi(f)$ on the orbifold $\mathcal{M}(\mathbb{C})$. This function is smooth on the complement of $\mathcal{Z}(f)$, and has a logarithmic singularity along $\mathcal{Z}(f)$ in the sense that for any local equation $\Psi(z)=0$ defining $\mathcal{Z}(f)(\mathbb{C})$, the function

$$
\Phi(f, z)+\log |\Psi(z)|^{2}
$$

on $\mathcal{M}(\mathbb{C}) \backslash \mathcal{Z}(f)(\mathbb{C})$ has a smooth extension across $\mathcal{Z}(f)(\mathbb{C})$.

Remark 3.2.1. Amazingly, the regularized integral defining $\Phi(f, z, g)$ converges at every point of $\mathcal{D}$, and so the function $\Phi(f)$ has a well-defined value even at points of the divisor $\mathcal{Z}(f)(\mathbb{C})$. The 


\section{Height PAIRINGs on orthogonal Shimura varieties}

value of the discontinuous function $\Phi(f)$ at the points of $\mathcal{Z}(f)(\mathbb{C})$ will play a key role in our later calculations of improper intersection.

Among the harmonic weak Maass forms of weight $1-n / 2$ are the Hejhal-Poincarè series

$$
F_{m, \mu}(\tau) \in H_{1-\frac{n}{2}}\left(\omega_{L}\right)
$$

defined for all $\mu \in L^{\vee} / L$ and positive $m \in \mathbb{Z}+Q(\mu)$, which may be rescaled to have holomorphic parts

$$
F_{m, \mu}^{+}(\tau)=\frac{1}{2}\left(q^{-m} \varphi_{\mu}+q^{-m} \varphi_{-\mu}\right)+O(1)
$$

In particular $\mathcal{Z}\left(F_{m, \mu}\right)=\mathcal{Z}(m, \mu)$. See [Bru02, Def. $1.8 \&$ Prop. 1.10] and [BF04, Remark 3.10] for details.

\subsection{Eisenstein series and the Rankin-Selberg integral}

Fix a rational negative 2-plane $V_{0} \subset V$, and let $L_{0}=V_{0} \cap L$. We assume that $C^{+}\left(L_{0}\right)$ is the maximal order in the quadratic imaginary field $C^{+}\left(V_{0}\right)$. This implies, in particular, that $L_{0}$ is a maximal quadratic space. Let

$$
\Lambda=\left\{x \in L: x \perp L_{0}\right\}
$$

so that $L_{0} \oplus \Lambda \subset L$ with finite index, and $\Lambda$ is also a maximal quadratic space.

Let $\mathfrak{S}_{\Lambda}$ be the space of complex-valued functions on $\Lambda^{\vee} / \Lambda$, and let $\omega_{\Lambda}: \widetilde{\mathrm{SL}_{2}}(\mathbb{Z}) \rightarrow \operatorname{Aut}\left(\mathfrak{S}_{\Lambda}\right)$ be the Weil representation. Let $\mathfrak{S}_{\Lambda}^{\vee}$ be the complex linear dual of $\mathfrak{S}_{\Lambda}$, and for each $m \in \mathbb{Q}$ define the representation number $R_{\Lambda}(m) \in \mathfrak{S}_{\Lambda}^{\vee}$ by

$$
R_{\Lambda}(m, \varphi)=\sum_{\substack{x \in \Lambda^{\vee} \\ Q(x)=m}} \varphi(x)
$$

for any $\varphi \in \mathfrak{S}_{\Lambda}$. As in $[\mathrm{BY} 09, \S 2]$, these representation numbers are the Fourier coefficients of a vector-valued modular form

$$
\Theta_{\Lambda}(\tau)=\sum_{m \in \mathbb{Q}} R_{\Lambda}(m) q^{m} \in M_{\frac{n}{2}}\left(\omega_{\Lambda}^{\vee}\right)
$$

Abbreviate $R_{\Lambda}(m, \mu)=R_{\Lambda}\left(m, \varphi_{\mu}\right)$, where $\varphi_{\mu} \in \mathfrak{S}_{\Lambda}$ is the characteristic function of $\mu \in \Lambda^{\vee} / \Lambda$, so that

$$
R_{\Lambda}(m, \mu)=\#\{x \in \mu+\Lambda: Q(x)=m\} .
$$

Let $F(\tau)$ be a cuspidal modular form

$$
F(\tau)=\sum_{m \in \mathbb{Q}>0} b_{F}(m) q^{m} \in S_{1+\frac{n}{2}}\left(\bar{\omega}_{L}\right) .
$$

Using the natural inclusion $L^{\vee} \subset L_{0}^{\vee} \oplus \Lambda^{\vee}$, every function on $L^{\vee}$ extends to a function on $L_{0}^{\vee} \oplus \Lambda^{\vee}$ identically zero off of $L^{\vee}$. Thus, there is a natural "extension by zero" map

$$
\mathfrak{S}_{L} \rightarrow \mathfrak{S}_{L_{0} \oplus \Lambda}
$$

which allows us to view $\overline{b_{F}(m)} \in \mathfrak{S}_{L_{0} \oplus \Lambda}$. The inclusion $\Lambda^{\vee} \rightarrow L_{0}^{\vee} \oplus \Lambda^{\vee}$ defines a restriction of functions homomorphism $\mathfrak{S}_{L_{0} \oplus \Lambda} \rightarrow \mathfrak{S}_{\Lambda}$. Using this, we can view $R_{\Lambda}(m) \in \mathfrak{S}_{L_{0} \oplus \Lambda}^{\vee}$. This allows us to define the Rankin-Selberg convolution L-function

$$
L\left(F, \Theta_{\Lambda}, s\right)=\Gamma\left(\frac{s+n}{2}\right) \sum_{m \in \mathbb{Q}^{+}} \frac{\left\{\overline{b_{F}(m)}, R_{\Lambda}(m)\right\}}{(4 \pi m)^{(s+n) / 2}},
$$




\section{F. Andreatta, E. Z. Goren, B. Howard, K. Madapusi Pera}

in which the pairing on the right is the tautological pairing

$$
\{\cdot, \cdot\}: \mathfrak{S}_{L_{0} \oplus \Lambda} \times \mathfrak{S}_{L_{0} \oplus \Lambda}^{\vee} \rightarrow \mathbb{C} .
$$

Define an $\mathfrak{S}_{L_{0}}^{\vee}$-valued Eisenstein series $E_{L_{0}}(\tau, s)$ by

$$
E_{L_{0}}(\tau, s, \varphi)=\sum_{\gamma \in B \backslash \mathrm{SL}_{2}(\mathbb{Z})}\left(\omega_{L_{0}}(\gamma) \varphi\right)(0) \cdot \frac{\operatorname{Im}(\gamma \tau)^{s / 2}}{c \tau+d}
$$

for all $\varphi \in \mathfrak{S}_{L_{0}}$, where $B \subset \mathrm{SL}_{2}(\mathbb{Z})$ is the subset of upper triangular matrices, and $\omega_{L_{0}}$ is the Weil representation of $\mathrm{SL}_{2}(\mathbb{Z})$ on $\mathfrak{S}_{L_{0}}$. The Eisenstein series $E_{L_{0}}(\tau, s)$, which is precisely the incoherent Eisenstein series denoted $E_{L_{0}}(\tau, s ; 1)$ in [BY09, $\left.§ 2.2\right]$, transforms in the variable $\tau$ like a weight 1 modular form of representation $\omega_{L_{0}}^{\vee}$. It is initially defined for $\operatorname{Re}(s) \gg 0$, but has meromorphic continuation to all $s$, and vanishes at $s=0$. The usual Rankin-Selberg unfolding method shows that

$$
L\left(F, \Theta_{\Lambda}, s\right)=\int_{\mathrm{SL}_{2}(\mathbb{Z}) \backslash \mathcal{H}}\left\{\bar{F}, E_{L_{0}}(s) \otimes \Theta_{\Lambda}\right\} \frac{d u d v}{v^{1-\frac{n}{2}}},
$$

where the pairing on the right is (3.3), and we are using the isomorphism

$$
\mathfrak{S}_{L_{0}}^{\vee} \otimes \mathfrak{S}_{\Lambda}^{\vee} \stackrel{\simeq}{\rightarrow} \mathfrak{S}_{L_{0} \oplus \Lambda}^{\vee}
$$

to view $E_{L_{0}}(s) \otimes \Theta_{\Lambda}$ as a smooth modular form valued in $\mathfrak{S}_{L_{0} \oplus \Lambda}^{\vee}$.

The central derivative $E_{L_{0}}^{\prime}(\tau, 0)$ is a harmonic $\mathfrak{S}_{L_{0}}^{\vee}$-valued function transforming like a weight 1 modular form of representation $\omega_{L_{0}}^{\vee}$. Denote the holomorphic part of the central derivative by

$$
\mathcal{E}_{L_{0}}(\tau)=\sum_{m \in \mathbb{Q}} a_{L_{0}}^{+}(m) \cdot q^{m}
$$

We will sometimes abbreviate $a_{L_{0}}^{+}(m, \mu)=a_{L_{0}}^{+}\left(m, \varphi_{\mu}\right)$, where $\varphi_{\mu} \in \mathfrak{S}_{L_{0}}$ is the characteristic function of $\mu \in L_{0}^{\vee} / L_{0}$. Note that our $a_{L_{0}}^{+}(m, \mu)$ is exactly the $\kappa(m, \mu)$ of [BY09, (2.25)].

Abbreviate $\boldsymbol{k}=C^{+}\left(V_{0}\right)$, and recall that $\boldsymbol{k}$ is a quadratic imaginary field. For any positive rational number $m$, define a finite set of primes

$$
\operatorname{Diff}_{L_{0}}(m)=\left\{\text { primes } p<\infty: L_{0} \otimes_{\mathbb{Z}} \mathbb{Q}_{p} \text { does not represent } m\right\} .
$$

Note that all primes in this set are non-split in $\boldsymbol{k}$. Because $L_{0}$ is negative definite, the set $\operatorname{Diff}_{L_{0}}(m)$ has odd cardinality; in particular, Diff $L_{0}(m) \neq \emptyset$. For $m \in \mathbb{Z}_{>0}$, set

$$
\rho(m)=\#\left\{\text { nonzero ideals } \mathfrak{m} \subset \mathcal{O}_{\boldsymbol{k}}: \mathrm{N}(\mathfrak{m})=m\right\} .
$$

We will extend $\rho$ to a function on all rational numbers by setting $\rho(m)=0$ for all $m \in \mathbb{Q} \backslash \mathbb{Z}_{>0}$.

The coefficients $a_{L_{0}}^{+}(m)$ were computed by Schofer [Sch09], but those formulas contain a minor misstatement. This misstatement was corrected when Schofer's formulas were reproduced in [BY09, Theorem 2.6], but the formula for the constant term $a_{L_{0}}^{+}(0)$ in [BY09] is misstated ${ }^{3}$. We record now the corrected formulas.

Proposition 3.3.1 Schofer. Let $d_{\boldsymbol{k}}, h_{\boldsymbol{k}}$, and $w_{\boldsymbol{k}}$ be the discriminant of $\boldsymbol{k}$, the class number of $\boldsymbol{k}$, and number of roots of unity in $\boldsymbol{k}$, respectively, and assume that $d_{\boldsymbol{k}}$ is odd. The coefficients $a_{L_{0}}^{+}(m)$ are as follows:

\footnotetext{
${ }^{3}$ The formula for $a_{L_{0}}^{+}(0,0)=\kappa(0,0)$ in [BY09] appears to agree with the formula in [Sch09]. In fact it does not, as the two papers use different normalizations for the completed $L$-function $\Lambda(\chi, s)$. It is Schofer's formula that is correct.
} 
(i) The constant term is

$$
a_{L_{0}}^{+}(0, \varphi)=\varphi(0) \cdot\left(\gamma+\log \left|\frac{4 \pi}{d_{\boldsymbol{k}}}\right|-2 \frac{L^{\prime}\left(\chi_{\boldsymbol{k}}, 0\right)}{L\left(\chi_{\boldsymbol{k}}, 0\right)}\right) .
$$

Here $\gamma=-\Gamma^{\prime}(1)$ is the Euler-Mascheroni constant, and $\chi_{\boldsymbol{k}}$ is the quadratic Dirichlet character associated with $\boldsymbol{k} / \mathbb{Q}$.

(ii) If $m>0$ and $\operatorname{Diff}_{L_{0}}(m)=\{p\}$ for a single prime $p$, then

$$
a_{L_{0}}^{+}(m, \varphi)=-\frac{w_{\boldsymbol{k}}}{2 h_{\boldsymbol{k}}} \cdot \rho\left(\frac{m\left|d_{\boldsymbol{k}}\right|}{p^{\epsilon}}\right) \cdot \operatorname{ord}_{p}(p m) \cdot \log (p) \sum_{\substack{\mu \in L_{0}^{\vee} / L_{0} \\ Q(\mu)=m}} 2^{s(\mu)} \varphi(\mu) .
$$

On the right hand side, $s(\mu)$ is the number of primes $\ell \mid \operatorname{disc}\left(L_{0}\right)$ such that $\mu \in L_{0, \ell}$,

$$
\epsilon=\left\{\begin{array}{l}
1 \quad \text { if } p \text { is inert in } \boldsymbol{k} \\
0 \quad \text { if } p \text { is ramified in } \boldsymbol{k},
\end{array}\right.
$$

and $Q(\mu)=m$ is understood as an equality in $\mathbb{Q} / \mathbb{Z}$.

(iii) If $m>0$ and $\left|\operatorname{Diff}_{L_{0}}(m)\right|>1$, or if $m<0$, then $a_{L_{0}}^{+}(m)=0$.

\section{Complex multiplication cycles}

Keep $L_{0} \oplus \Lambda \subset L$ as in $\S 3.3$, so that $L$ is a maximal lattice of signature $(n, 2), L_{0}$ is a sublattice of signature $(0,2)$, and

$$
\Lambda=L_{0}^{\perp}=\left\{x \in L: x \perp L_{0}\right\} .
$$

Abbreviate $V_{0}=L_{0} \otimes_{\mathbb{Z}} \mathbb{Q}$ and $V=L \otimes_{\mathbb{Z}} \mathbb{Q}$. The even part $C^{+}\left(V_{0}\right)$ of the Clifford algebra is isomorphic to a quadratic imaginary field $\boldsymbol{k}$, and we continue to assume that $C^{+}\left(L_{0}\right)$ is its maximal order.

In this section we construct an $\mathcal{O}_{\boldsymbol{k}}$-stack $\mathcal{Y}$ from the quadratic space $L_{0}$. Assuming that the discriminant of $\boldsymbol{k}$ is odd, the inclusion $L_{0} \subset L$ induces a morphism $\mathcal{Y} \rightarrow \mathcal{M}_{\mathcal{O}_{\boldsymbol{k}}}$, where $\mathcal{M}$ is the stack over $\mathbb{Z}$ determined by the quadratic space $L$. We define special divisors on $\mathcal{Y}$, compute their degrees explicitly, and compare them with the special divisors on $\mathcal{M}$.

\subsection{Preliminaries}

Fix once and for all an isomorphism of $C^{+}\left(L_{0}\right)$ with the maximal order $\mathcal{O}_{\boldsymbol{k}}$ in a quadratic imaginary subfield $\boldsymbol{k} \subset \mathbb{C}$. Let $d_{\boldsymbol{k}}, h_{\boldsymbol{k}}$, and $w_{\boldsymbol{k}}$ be as in Proposition 3.3.1. The $\mathbb{Z} / 2 \mathbb{Z}$-grading on $C\left(L_{0}\right)$ takes the form

$$
C\left(L_{0}\right)=\mathcal{O}_{\boldsymbol{k}} \oplus L_{0},
$$

and $L_{0}$ is both a left and right $\mathcal{O}_{\boldsymbol{k}}$-module; the two actions are related by $x \alpha=\bar{\alpha} x$ for every $x \in L_{0}$ and $\alpha \in \mathcal{O}_{\boldsymbol{k}}$.

There is a fractional $\mathcal{O}_{\boldsymbol{k}}$-ideal $\mathfrak{a}$ and an isomorphism $L_{0} \stackrel{\simeq}{\rightarrow} \mathfrak{a}$ of left $\mathcal{O}_{\boldsymbol{k}}$-modules identifying the quadratic form on $L_{0}$ with the form $-\mathrm{N}(\cdot) / \mathrm{N}(\mathfrak{a})$ on $\mathfrak{a}$, and an easy exercise shows that

$$
L_{0}^{\vee}=\mathfrak{d}_{k}^{-1} L_{0},
$$

where $\mathfrak{d}_{\boldsymbol{k}}$ is the different of $\boldsymbol{k} / \mathbb{Q}$. 


\subsection{The 0-dimensional Shimura variety}

Let $T$ be the group scheme over $\mathbb{Z}$ with functor of points

$$
T(R)=\left(C^{+}\left(L_{0}\right) \otimes_{\mathbb{Z}} R\right)^{\times}
$$

for any $\mathbb{Z}$-algebra $R$. Thus $T_{\mathbb{Q}}=\operatorname{GSpin}\left(V_{0}\right)$ is a torus of rank 2 and can be identified with the maximal subgroup of $G$ that acts trivially on the subspace $\Lambda_{\mathbb{Q}} \subset V$. Set $K_{0}=T(\widehat{\mathbb{Z}})$.

Using our fixed embedding $\mathcal{O}_{\boldsymbol{k}} \subset \mathbb{C}$, left multiplication makes $V_{0, \mathbb{R}}$ into a complex vector space. This determines an orientation on $V_{0, \mathbb{R}}$, and the oriented negative plane $V_{0, \mathbb{R}} \subset V_{\mathbb{R}}$ determines a point $\boldsymbol{h}_{0} \in \mathcal{D}$. The isomorphism of (2.1) then associates to $\boldsymbol{h}_{0}$ an isotropic line in $V_{0, \mathbb{C}}$; explicitly, this is the line on which the right action of $\mathcal{O}_{\boldsymbol{k}}$ agrees with the inclusion $\mathcal{O}_{\boldsymbol{k}} \subset \mathbb{C}=\operatorname{End}_{\mathbb{C}}\left(V_{0, \mathbb{C}}\right)$.

The pair $\left(T_{\mathbb{Q}}, \boldsymbol{h}_{0}\right)$ is a Shimura datum with reflex field $\boldsymbol{k} \subset \mathbb{C}$, and the 0-dimensional complex orbifold

$$
Y(\mathbb{C})=T(\mathbb{Q}) \backslash\left\{\boldsymbol{h}_{0}\right\} \times T\left(\mathbb{A}_{f}\right) / K_{0}
$$

is the complex fiber of a $\boldsymbol{k}$-stack $Y$. As in $\S 2.2$, the representation of $T_{\mathbb{Q}}$ on $V_{0}$ and the lattice

$$
L_{0} \otimes \widehat{\mathbb{Z}} \subset V_{0} \otimes \mathbb{A}_{f}
$$

determine a variation of polarized $\mathbb{Z}$-Hodge structures $\left(\boldsymbol{V}_{0, \mathrm{Be}}, \mathrm{Fil} \boldsymbol{V}_{0, \mathrm{dR}, Y(\mathbb{C})}\right)$ of type $(-1,1)$, $(1,-1)$ over $Y(\mathbb{C})$.

Similarly, the representation of $T_{\mathbb{Q}}$ on $C\left(V_{0}\right)$ by left multiplication, together with the lattice

$$
C\left(L_{0}\right)_{\widehat{\mathbb{Z}}} \subset C\left(V_{0}\right)_{\mathbb{A}_{f}},
$$

determine a variation of $\mathbb{Z}$-Hodge structures $\left(\boldsymbol{H}_{0, \mathrm{Be}}, \mathrm{Fil} \boldsymbol{H}^{\bullet} \boldsymbol{H}_{0, \mathrm{dR}, \mathcal{Y}(\mathbb{C})}\right)$ of type $(-1,0),(0,-1)$. As in $\S 2.3$, this is the homology of an abelian scheme

$$
\mathcal{A}_{0, Y} \rightarrow Y
$$

of relative dimension 2 , equipped with a right $C\left(L_{0}\right)$-action and a compatible $\mathbb{Z} / 2 \mathbb{Z}$-grading

$$
\mathcal{A}_{0, Y}=\mathcal{A}_{0, Y}^{+} \times \mathcal{A}_{0, Y}^{-} .
$$

As before, we will refer to this abelian scheme as the Kuga-Satake abelian scheme over $Y$.

\subsection{The integral model}

We now define integral models of $Y$ and $\mathcal{A}_{0, Y}$ over $\mathcal{O}_{\boldsymbol{k}}$.

Definition 4.3.1. If $S$ is an $\mathcal{O}_{\boldsymbol{k}^{-}}$-scheme, an $\mathcal{O}_{\boldsymbol{k}}$-elliptic curve over $S$ is an elliptic curve $\mathcal{E} \rightarrow S$ endowed with an action of $\mathcal{O}_{k}$ in such a way that the induced action on the $\mathcal{O}_{S}$-module $\operatorname{Lie}(\mathcal{E})$ is through the structure morphism $\mathcal{O}_{k} \rightarrow \mathcal{O}_{S}$.

Remark 4.3.2. Our convention is that the $\mathcal{O}_{\boldsymbol{k}}$-action on any $\mathcal{O}_{\boldsymbol{k}}$-elliptic curve is written on the right.

Denote by $\mathcal{Y}$ the $\mathcal{O}_{\boldsymbol{k}}$-stack classifying $\mathcal{O}_{\boldsymbol{k}}$-elliptic curves over $\mathcal{O}_{\boldsymbol{k}}$-schemes. Every point $y \in$ $\mathcal{Y}(\mathbb{C})$ has automorphism group $\mathcal{O}_{k}^{\times}$, and

$$
\sum_{y \in \mathcal{Y}(\mathbb{C})} \frac{1}{\# \operatorname{Aut}(y)}=\frac{h_{\boldsymbol{k}}}{w_{\boldsymbol{k}}} .
$$

As in [KRY99, Proposition 5.1], the stack $\mathcal{Y}$ is finite étale over $\mathcal{O}_{\boldsymbol{k}}$. In particular $\mathcal{Y}$ is regular of dimension 1 , and is flat over $\mathcal{O}_{\boldsymbol{k}}$. 
The positive graded part $\mathcal{A}_{0, Y}^{+}$of the Kuga-Satake abelian scheme, with its right action of $C^{+}\left(L_{0}\right)=\mathcal{O}_{\boldsymbol{k}}$, is an $\mathcal{O}_{\boldsymbol{k}}$-elliptic curve over $Y$ (for the obvious extension of this notion over algebraic stacks.) Therefore, it defines a morphism $Y \rightarrow \mathcal{Y}$. By checking on complex points, we see that this map defines an isomorphism $Y \stackrel{\simeq}{\rightarrow} \mathcal{Y}_{\boldsymbol{k}}$. Again by checking on complex points, one can show that the natural map

$$
\mathcal{A}_{0, Y}^{+} \otimes \mathcal{O}_{k} C\left(L_{0}\right) \rightarrow \mathcal{A}_{0, Y}
$$

is an isomorphism, from which it follows that

$$
\mathcal{A}_{0, Y}^{-} \stackrel{\simeq}{\rightarrow} \mathcal{A}_{0, Y}^{+} \otimes_{\mathcal{O}_{k}} L_{0}
$$

From these observations, we can use the universal $\mathcal{O}_{\boldsymbol{k}}$-elliptic curve $\mathcal{E} \rightarrow \mathcal{Y}$ to extend the Kuga-Satake abelian scheme $\mathcal{A}_{0, Y} \rightarrow Y$ to an abelian scheme

$$
\mathcal{A}_{0}=\mathcal{E} \otimes \mathcal{O}_{\boldsymbol{k}} C\left(L_{0}\right)
$$

over $\mathcal{Y}$ of relative dimension 2. By construction, $\mathcal{A}_{0}$ carries a right action of $C\left(L_{0}\right)$ and a $\mathbb{Z} / 2 \mathbb{Z}$ grading $\mathcal{A}_{0}=\mathcal{A}_{0}^{+} \times \mathcal{A}_{0}^{-}$induced by (4.1), where $\mathcal{A}_{0}^{+}=\mathcal{E}$ and $\mathcal{A}_{0}^{-}=\mathcal{E} \otimes_{\mathcal{O}_{k}} L_{0}$.

Remark 4.3.3. Note that the tensor product $\mathcal{E} \otimes \mathcal{O}_{\boldsymbol{k}} L_{0}$ is with respect to the left action of $\mathcal{O}_{\boldsymbol{k}}$ on $L_{0}$, and $\mathcal{O}_{\boldsymbol{k}} \subset C\left(L_{0}\right)$ acts on $\mathcal{E} \otimes_{\mathcal{O}_{k}} L_{0}$ through right multiplication on $L_{0}$. From the relation $x \alpha=\bar{\alpha} x$ for $x \in L_{0}$ and $\alpha \in \mathcal{O}_{\boldsymbol{k}}$, it follows that $\mathcal{O}_{\boldsymbol{k}}$ acts on $\operatorname{Lie}\left(\mathcal{A}_{0}^{-}\right)$through the conjugate of the structure morphism $\mathcal{O}_{k} \rightarrow \mathcal{O}_{\mathcal{Y}}$.

There are obvious analogues on $\mathcal{Y}$ of the sheaves $\boldsymbol{H}$ defined in $\S 2.4$. Denote by $\boldsymbol{H}_{0, \mathrm{dR}}$ the first de Rham homology of $\mathcal{A}_{0}$ relative to $\mathcal{Y}$. Similarly, denote by $\boldsymbol{H}_{0, p \text {, et }}$ the first $p$-adic étale homology of $\mathcal{A}_{0}$ relative to $\mathcal{Y}[1 / p]$, and by $\boldsymbol{H}_{0, \text { crys }}$ the first crystalline homology of the special fiber of $\mathcal{A}_{0}$ at a prime $p$ relative to $\mathcal{Y}_{\mathbb{F}_{p}}$. These sheaves come with right actions of $C\left(L_{0}\right)$, and $\mathbb{Z} / 2 \mathbb{Z}$-gradings $\boldsymbol{H}_{0}=\boldsymbol{H}_{0}^{+} \times \boldsymbol{H}_{0}^{-}$in such a way that $\boldsymbol{H}_{0}^{-}=\boldsymbol{H}_{0}^{+} \otimes_{\mathcal{O}_{k}} L_{0}$. As in the higher dimensional case, over $\mathcal{Y}(\mathbb{C})$ the de Rham and étale sheaves arise from the Betti realization $\boldsymbol{H}_{0, \mathrm{Be}}$.

Define

$$
\boldsymbol{V}_{0} \subset \underline{\operatorname{End}}_{C\left(L_{0}\right)}\left(\boldsymbol{H}_{0}\right)
$$

as the submodule of grade shifting endomorphisms. As can be checked from the complex uniformization, over $\mathcal{Y}(\mathbb{C})$ this definition recovers the local system $\boldsymbol{V}_{0, \text { Be }}$ (for the Betti realization) and the vector bundle $\boldsymbol{V}_{0, \mathrm{dR}, Y(\mathbb{C})}$ (for the de Rham realization.) Indeed, this amounts to the fact that the embedding of $L_{0}$ into $\operatorname{End}\left(C\left(L_{0}\right)\right)$ via left multiplication identifies it with the space of grade shifting endomorphisms of $C\left(L_{0}\right)$ that commute with right multiplication by $C\left(L_{0}\right)$.

Restriction to $\boldsymbol{H}_{0}^{+}$defines an isomorphism

$$
\boldsymbol{V}_{0} \stackrel{\simeq}{\rightarrow} \underline{\operatorname{Hom}}_{\mathcal{O}_{k}}\left(\boldsymbol{H}_{0}^{+}, \boldsymbol{H}_{0}^{-}\right) .
$$

As $\boldsymbol{H}_{0}^{ \pm}$is a locally free $\mathcal{O}_{\boldsymbol{k}} \otimes \mathbf{1}$-module of rank one, the same is true of $\boldsymbol{V}_{0}$. In particular, $\boldsymbol{V}_{0}$ is a locally free 1-module of rank two. In the de Rham case, the Hodge filtration on $\boldsymbol{H}_{0, \mathrm{dR}}$ determines a submodule $\boldsymbol{V}_{0, \mathrm{dR}}^{1} \subset \boldsymbol{V}_{0, \mathrm{dR}}$ by

$$
\boldsymbol{V}_{0, \mathrm{dR}}^{1}=\boldsymbol{V}_{0, \mathrm{dR}} \cap \mathrm{Fil}^{1} \underline{\underline{\operatorname{End}}}\left(\boldsymbol{H}_{0, \mathrm{dR}}\right) .
$$

Definition 4.3.4. As in Definition 2.4.7, the line bundle $\boldsymbol{V}_{0, \mathrm{dR}}^{1} \subset \boldsymbol{V}_{0, \mathrm{dR}}$ is the tautological bundle on $\mathcal{Y}$, and its dual is the cotautological bundle.

The following proposition makes the cotautological bundle more explicit. 
Proposition 4.3.5. There are canonical isomorphisms of $\mathcal{O}_{\mathcal{Y}}$-modules

$$
\left(\boldsymbol{V}_{0, \mathrm{dR}}^{1}\right)^{\vee} \stackrel{\simeq}{\rightarrow} \operatorname{Lie}\left(\mathcal{A}_{0}^{+}\right) \otimes \operatorname{Lie}\left(\mathcal{A}_{0}^{-}\right) \stackrel{\simeq}{\rightarrow} \operatorname{Lie}(\mathcal{E})^{\otimes 2} \otimes \boldsymbol{L}_{0},
$$

where $\boldsymbol{L}_{0}=\mathcal{O}_{\mathcal{Y}} \otimes_{\mathcal{O}_{k}} L_{0}$.

Proof. We have canonical isomorphisms of line bundles over $\mathcal{Y}$

$$
\begin{aligned}
& \boldsymbol{H}_{0, \mathrm{dR}}^{+} / \mathrm{Fil}^{0} \boldsymbol{H}_{0, \mathrm{dR}}^{+} \stackrel{\simeq}{\leftrightarrows} \operatorname{Lie}\left(\mathcal{A}_{0}^{+}\right) \\
& \boldsymbol{H}_{0, \mathrm{dR}}^{-} / \mathrm{Fil}^{0} \boldsymbol{H}_{0, \mathrm{dR}}^{-} \stackrel{\simeq}{\longrightarrow} \operatorname{Lie}\left(\mathcal{A}_{0}^{-}\right),
\end{aligned}
$$

and the (de Rham realization of the) unique principal polarization on $\mathcal{A}_{0}^{-}$induces an isomorphism of $\mathcal{O}_{\mathcal{y}}$-modules

$$
\mathrm{Fil}^{0} \boldsymbol{H}_{0, \mathrm{dR}}^{-} \stackrel{\simeq}{\rightarrow}\left(\boldsymbol{H}_{0, \mathrm{dR}}^{-} / \mathrm{Fil}^{0} \boldsymbol{H}_{0, \mathrm{dR}}^{-}\right)^{\vee}=\operatorname{Lie}\left(\mathcal{A}_{0}^{-}\right)^{\vee} .
$$

The isomorphism (4.3) restricts to

$$
\begin{aligned}
\boldsymbol{V}_{0, \mathrm{dR}}^{1} & \stackrel{\simeq}{\rightarrow} \operatorname{Fil}^{1} \underline{\operatorname{Hom}}_{\mathcal{O}_{\boldsymbol{k}}}\left(\boldsymbol{H}_{0, \mathrm{dR}}^{+}, \boldsymbol{H}_{0, \mathrm{dR}}^{-}\right) \\
& \stackrel{\simeq}{\rightarrow} \underline{\operatorname{Hom}}_{\mathcal{O}_{\boldsymbol{k}}}\left(\boldsymbol{H}_{0, \mathrm{dR}}^{+} / \mathrm{Fil}^{0} \boldsymbol{H}_{0, \mathrm{dR}}^{+}, \operatorname{Fil}^{0} \boldsymbol{H}_{0, \mathrm{dR}}^{-}\right) \\
& \stackrel{\simeq}{\operatorname{Hom}}\left(\operatorname{Lie}\left(\mathcal{A}_{0}^{+}\right), \operatorname{Lie}\left(\mathcal{A}_{0}^{-}\right)^{\vee}\right),
\end{aligned}
$$

and dualizing yields the first isomorphism in (4.4). The second isomorphism is clear from the definition of $\mathcal{A}_{0}^{ \pm}$and the relation $\mathcal{A}_{0}^{-}=\mathcal{E} \otimes_{\mathcal{O}_{k}} L_{0}$.

Remark 4.3.6. It is clear from (4.5) that the isotropic line $\boldsymbol{V}_{0, \mathrm{dR}}^{1} \subset \boldsymbol{V}_{0, \mathrm{dR}}$ is stable under the action of $\mathcal{O}_{\boldsymbol{k}}$ induced by (4.3), and that $\mathcal{O}_{\boldsymbol{k}}$ acts on this line through the structure morphism $\mathcal{O}_{k} \rightarrow \mathcal{O}_{\mathcal{Y}}$

\subsection{Special endomorphisms}

If $\mathcal{E}_{1}$ and $\mathcal{E}_{2}$ are any elliptic curves with right actions of $\mathcal{O}_{\boldsymbol{k}}$, we make $\operatorname{Hom}\left(\mathcal{E}_{1}, \mathcal{E}_{2}\right)$ into a right $\mathcal{O}_{\boldsymbol{k}}$-module via

$$
(f \cdot \alpha)(-)=f(-) \cdot \alpha
$$

for all $f \in \operatorname{Hom}\left(\mathcal{E}_{1}, \mathcal{E}_{2}\right)$ and $\alpha \in \mathcal{O}_{\boldsymbol{k}}$. With this convention, for any $\mathcal{Y}$-scheme $S$ there is an isomorphism of right $\mathcal{O}_{\boldsymbol{k}}$-modules

$$
\operatorname{Hom}\left(\mathcal{E}_{S}, \mathcal{E}_{S} \otimes_{\mathcal{O}_{k}} L_{0}\right) \stackrel{\simeq}{\rightarrow} \operatorname{End}\left(\mathcal{E}_{S}\right) \otimes_{\mathcal{O}_{k}} L_{0} .
$$

This restricts to an isomorphism

$$
\operatorname{Hom}_{\mathcal{O}_{\boldsymbol{k}}}\left(\mathcal{E}_{S}, \mathcal{E}_{S} \otimes_{\mathcal{O}_{\boldsymbol{k}}} L_{0}\right) \stackrel{\simeq}{\rightarrow} \operatorname{End}_{\overline{\mathcal{O}}_{\boldsymbol{k}}}\left(\mathcal{E}_{S}\right) \otimes \mathcal{O}_{\boldsymbol{k}} L_{0},
$$

which we rewrite as

$$
\operatorname{Hom}_{\mathcal{O}_{k}}\left(\mathcal{A}_{0, S}^{+}, \mathcal{A}_{0, S}^{-}\right) \stackrel{\simeq}{\rightarrow} \operatorname{End}_{\overline{\mathcal{O}}_{k}}\left(\mathcal{E}_{S}\right) \otimes_{\mathcal{O}_{k}} L_{0}
$$

Here the subscripts $\mathcal{O}_{\boldsymbol{k}}$ and $\overline{\mathcal{O}}_{\boldsymbol{k}}$ indicate $\mathcal{O}_{\boldsymbol{k}}$-linear and $\mathcal{O}_{\boldsymbol{k}}$-conjugate-linear maps, respectively. The quadratic form deg on the left hand side of (4.6) corresponds to the quadratic form

$$
x \otimes v \mapsto-\operatorname{deg}(x) \cdot Q(v)
$$

on the right hand side (which is positive definite, as deg and $-Q$ are positive definite.)

Elements of $\operatorname{End}_{\overline{\mathcal{O}}_{k}}\left(\mathcal{E}_{S}\right) \otimes_{\mathcal{O}_{k}} V_{0}$ are special quasi-endomorphisms of $\mathcal{E}_{S}$. For every $\mu \in L_{0}^{\vee}$ define a collection of special quasi-endomorphisms by

$$
V_{\mu}\left(\mathcal{E}_{S}\right)=\left\{x \in \operatorname{End}_{\overline{\mathcal{O}}_{k}}\left(\mathcal{E}_{S}\right) \otimes_{\mathcal{O}_{k}} L_{0}^{\vee}: x-\mathrm{id} \otimes \mu \in \operatorname{End}\left(\mathcal{E}_{S}\right) \otimes_{\mathcal{O}_{k}} L_{0}\right\} .
$$




\section{Height PAIRINGs on ORTHOgOnAL Shimura VARIETIES}

This collection depends only on the coset $\mu \in L_{0}^{\vee} / L_{0}$. We will sometimes abbreviate

$$
V\left(\mathcal{E}_{S}\right)=\operatorname{End}_{\overline{\mathcal{O}}_{\boldsymbol{k}}}\left(\mathcal{E}_{S}\right) \otimes_{\mathcal{O}_{\boldsymbol{k}}} L_{0}
$$

for the space defined by the coset $\mu=0$.

Remark 4.4.1. For any $S \rightarrow \mathcal{Y}$ as above, let $V\left(\mathcal{A}_{0, S}\right) \subset \operatorname{End}\left(\mathcal{A}_{0, S}\right)$ be the submodule of $C\left(L_{0}\right)$ linear grade-shifting endomorphisms of $\mathcal{A}_{0, S}$. Equivalently, $x \in \operatorname{End}\left(\mathcal{A}_{0, S}\right)$ lies in $V\left(\mathcal{A}_{0, S}\right)$ if and only if all of its homological realizations lie in the subsheaf $\boldsymbol{V}_{0} \subset \underline{\operatorname{End}}\left(\boldsymbol{H}_{0}\right)$. Restriction to the positive graded part identifies

$$
V\left(\mathcal{A}_{0, S}\right) \stackrel{\simeq}{\rightarrow} \operatorname{Hom}_{\mathcal{O}_{\boldsymbol{k}}}\left(\mathcal{A}_{0, S}^{+}, \mathcal{A}_{0, S}^{-}\right) \stackrel{\simeq}{\rightarrow} \operatorname{End}_{\overline{\mathcal{O}}_{\boldsymbol{k}}}\left(\mathcal{E}_{S}\right) \otimes_{\mathcal{O}_{\boldsymbol{k}}} L_{0} \stackrel{\simeq}{\rightarrow} V\left(\mathcal{E}_{S}\right),
$$

where the middle isomorphism is (4.6).

\subsection{Degrees of special divisors}

In this subsection we define the special divisors $\mathcal{Z}_{0}(m, \mu) \rightarrow \mathcal{Y}$ and compute their degrees.

For a non-negative rational number $m$ and a class $\mu \in L_{0}^{\vee} / L_{0}$, define $\mathcal{Z}_{0}(m, \mu)$ to be the $\mathcal{O}_{\boldsymbol{k}^{-}}$ stack that assigns to an $\mathcal{O}_{\boldsymbol{k}}$-scheme $S$ the groupoid of pairs $\left(\mathcal{E}_{S}, x\right)$ in which $\mathcal{E}_{S}$ is an $\mathcal{O}_{\boldsymbol{k}}$-elliptic curve over $S$, and $x \in V_{\mu}\left(\mathcal{E}_{S}\right)$ satisfies $Q(x)=m$. Note that for $m=0$ we have

$$
\mathcal{Z}_{0}(0, \mu)= \begin{cases}\emptyset & \text { if } \mu \neq 0 \\ \mathcal{Y} & \text { if } \mu=0\end{cases}
$$

The following theorem is due to Kudla-Rapoport-Yang [KRY99] in the special case where $\boldsymbol{k}$ has prime discriminant and $\mu=0$. Subsequent generalizations and variants can be found in [KY13], [BHY15], and [How12].

Theorem 4.5.1. Assume that $d_{\boldsymbol{k}}$ is odd, and recall the finite set of primes $\operatorname{Diff}_{L_{0}}(m)$ of (3.4). For $m>0$ the stack $\mathcal{Z}_{0}(m, \mu)$ has the following properties:

(i) If $\left|\operatorname{Diff}_{L_{0}}(m)\right|>1$, or if $Q(\mu) \neq m$ in $\mathbb{Q} / \mathbb{Z}$, then $\mathcal{Z}_{0}(m, \mu)=\emptyset$.

(ii) If $\operatorname{Diff}_{L_{0}}(m)=\{p\}$ and $Q(\mu)=m$ in $\mathbb{Q} / \mathbb{Z}$, then $\mathcal{Z}_{0}(m, \mu)$ has dimension 0 and is supported in characteristic $p$. Furthermore,

$$
\frac{\log (\mathrm{N}(\mathfrak{p}))}{\log (p)} \sum_{z \in \mathcal{Z}_{0}(m, \mu)\left(\overline{\mathbb{F}}_{\mathfrak{p}}\right)} \frac{\operatorname{length}\left(\mathcal{O}_{\mathcal{Z}_{0}(m, \mu), z}\right)}{\# \operatorname{Aut}(z)}=2^{s(\mu)-1} \cdot \operatorname{ord}_{p}(p m) \cdot \rho\left(\frac{m\left|d_{\boldsymbol{k}}\right|}{p^{\epsilon}}\right),
$$

where $\mathfrak{p}$ is the unique prime of $\boldsymbol{k}$ above $p$, and $\epsilon, \rho$, and $s(\mu)$ are as in Proposition 3.3.1.

In either case

$$
\sum_{\mathfrak{p} \subset \mathcal{O}_{k}} \log (\mathrm{N}(\mathfrak{p})) \sum_{z \in \mathcal{Z}_{0}(m, \mu)\left(\overline{\mathbb{F}}_{\mathfrak{p}}\right)} \frac{\operatorname{length}\left(\mathcal{O}_{\mathcal{Z}_{0}(m, \mu), z}\right)}{\# \operatorname{Aut}(z)}=-\frac{h_{\boldsymbol{k}}}{w_{\boldsymbol{k}}} \cdot a_{L_{0}}(m, \mu) .
$$

Proof. Let $\mathfrak{p}$ be a prime of $\mathcal{O}_{\boldsymbol{k}}$, and let $p$ be the rational prime below it. Suppose we have a point

$$
z=(\mathcal{E}, x) \in \mathcal{Z}_{0}(m, \mu)\left(\overline{\mathbb{F}}_{\mathfrak{p}}\right) .
$$

The existence of $x \in \operatorname{End}_{\overline{\mathcal{O}}_{\boldsymbol{k}}}(\mathcal{E}) \otimes \mathcal{O}_{\boldsymbol{k}} L_{0}$ already implies that $\mathcal{E}$ is supersingular, $p$ is non-split in $\boldsymbol{k}$, and $\operatorname{End}(\mathcal{E})_{\mathbb{Q}}$ is the quaternion division algebra ramified at $\infty$ and $p$.

As $\mathcal{O}_{\boldsymbol{k}} \hookrightarrow \operatorname{End}(E)$ we can extend this to an inclusion $\boldsymbol{k} \hookrightarrow \operatorname{End}(E)_{\mathbb{Q}}$. Since $\boldsymbol{k}=\mathbb{Q}\left(\sqrt{d_{\boldsymbol{k}}}\right)$ there is an $\eta \in \mathbb{Q}^{\times}$such that

$$
\operatorname{End}(E)_{\mathbb{Q}}=\left(\frac{d_{\boldsymbol{k}}, \eta}{\mathbb{Q}}\right)
$$




\section{F. Andreatta, E. Z. Goren, B. Howard, K. Madapusi Pera}

Consequently $\operatorname{End}_{\overline{\mathcal{O}}_{\boldsymbol{k}}}(E)_{\mathbb{Q}}=\boldsymbol{k} \cdot j$, where $j^{2}=\eta$ and $j \alpha=\bar{\alpha} j, \forall \alpha \in \mathcal{O}_{\boldsymbol{k}}$. Further, End $\overline{\mathcal{O}}_{\boldsymbol{k}}(E)_{\mathbb{Q}}=$ $\boldsymbol{k} \cdot \boldsymbol{j}$ is a one dimensional $\boldsymbol{k}$-vector space equipped with the quadratic form deg, which is just the reduced norm of the quaternion algebra. As $j$ has reduced norm $-\eta$, we deduce that there is an isomorphism of quadratic spaces

$$
\operatorname{End}_{\overline{\mathcal{O}}_{\boldsymbol{k}}}(E)_{\mathbb{Q}} \cong W_{-\eta},
$$

where for every $\gamma \in \mathbb{Q}^{\times}$we let $W_{\gamma}=\boldsymbol{k}$ equipped with the quadratic form $Q(w)=\gamma w \bar{w}$.

As $\operatorname{End}(E)_{\mathbb{Q}}$ is a quaternion algebra ramified precisely at $p$ and $\infty$, it follows that $\eta$ is a local norm from $\boldsymbol{k}$ everywhere but at $p$ and $\infty$.

Recalling that $V_{0}=L_{0} \otimes_{\mathbb{Z}} \mathbb{Q}$, any choice of left $\boldsymbol{k}$-module generator $v \in V_{0}$ identifies $V_{0} \stackrel{\simeq}{\rightarrow} W_{\gamma}$, where $\gamma=Q(v)$. Recalling (4.7), we deduce that there an isomorphism of quadratic spaces

$$
\operatorname{End}_{\overline{\mathcal{O}}_{\boldsymbol{k}}}(\mathcal{E}) \otimes_{\mathcal{O}_{\boldsymbol{k}}} V_{0} \stackrel{\simeq}{\rightarrow} W_{\eta \gamma} .
$$

The existence of $x$ shows that the quadratic space on the left represents $m$ globally, and hence $\eta \gamma / m$ is a norm from $\boldsymbol{k}$. This implies that $\gamma / m$, hence $\gamma m$, is a norm from $\boldsymbol{k}$ everywhere locally except at $\infty$ and $p$, from which we deduce $\operatorname{Diff}_{L_{0}}(m)=\{p\}$. The equality $m=Q(\mu)$ in $\mathbb{Q} / \mathbb{Z}$ can be checked directly, but it also follows from (2.17) and Proposition 4.6.3.

We have now proved that $\mathcal{Z}_{0}(m, \mu)\left(\overline{\mathbb{F}}_{\mathfrak{p}}\right) \neq \emptyset$ only when $\operatorname{Diff}_{L_{0}}(m)=\{p\}$ and $m=Q(\mu)$ in $\mathbb{Q} / \mathbb{Z}$, and hence have proved (i). The proof of (ii) requires a lemma.

LEMma 4.5.2. The existence of a point (4.9) implies $\mu \in L_{0, p}$.

Proof. By (4.2) we may assume that $p$ is ramified in $\boldsymbol{k}$. Fix a generator $\delta_{\boldsymbol{k}} \in \mathfrak{d}_{\boldsymbol{k}}$, so that $L_{0}^{\vee}=$ $\delta_{\boldsymbol{k}}^{-1} L_{0}$. Let $E_{p}$ be the $p$-divisible group of $\mathcal{E}$ with its induced $\mathcal{O}_{\boldsymbol{k}}$-action.

If $\mu \notin L_{0, p}$ then $\mu \delta_{\boldsymbol{k}}$ generates $L_{0, p}$ as an $\mathcal{O}_{\boldsymbol{k}, p}$-module. If we use this generator to identify

$$
\operatorname{End}\left(E_{p}\right) \otimes_{\mathcal{O}_{k}} L_{0} \stackrel{\simeq}{\longrightarrow} \operatorname{End}\left(E_{p}\right)
$$

and multiply both sides of the relation $x-\mathrm{id} \otimes \mu \in \operatorname{End}\left(E_{p}\right) \otimes_{\mathcal{O}_{\boldsymbol{k}}} L_{0}$ on the right by $\delta_{\boldsymbol{k}}$, we obtain

$$
y-\mathrm{id} \in \operatorname{End}\left(E_{p}\right) \cdot \delta_{\boldsymbol{k}},
$$

where we have set $y=x \delta_{\boldsymbol{k}} \in \operatorname{End}_{\overline{\mathcal{O}}_{\boldsymbol{k}}}\left(E_{p}\right)$.

The ring $\operatorname{End}\left(E_{p}\right)$ is the maximal order in the division quaternion algebra over $\mathbb{Q}_{p}$, and this maximal order is a (noncommutative) discrete valuation ring with $\operatorname{End}\left(E_{p}\right) \cdot \delta_{\boldsymbol{k}}$ as its unique maximal ideal. Using $y v=\bar{v} y$ for all $v \in \mathcal{O}_{\boldsymbol{k}, p}$, an exercise with quaternion algebras shows that $y$ has reduced trace 0 . Taking the reduced trace of (4.11) now implies that $-2 \cdot$ id lies in the maximal ideal of $\operatorname{End}\left(E_{p}\right)$, which is absurd, as our assumption that $d_{\boldsymbol{k}}$ is odd implies $p>2$.

It follows from Lemma 4.5.2 and the Serre-Tate theorem that we may completely ignore $\mu$ in the deformation problem that computes the length of the étale local ring at $z$. Gross's results on canonical liftings [Gro86] therefore imply

$$
\frac{\log (\mathrm{N}(\mathfrak{p}))}{\log (p)} \cdot \operatorname{length}\left(\mathcal{O}_{\mathcal{Z}_{0}(m, \mu), z}\right)=\operatorname{ord}_{p}(p m)
$$

for every point (4.9).

If $\operatorname{ord}_{p}(m)<0$ then the relation $m=Q(\mu)$ in $\mathbb{Q} / \mathbb{Z}$ implies $\mu \notin L_{0, p}$, and hence, using Lemma 4.5.2, both sides of the desired equality in (ii) are equal to 0 . Thus it only remains to prove

$$
\sum_{z \in \mathcal{Z}_{0}(m, \mu)\left(\overline{\mathbb{F}}_{\mathfrak{p}}\right)} \frac{1}{\# \operatorname{Aut}(z)}=2^{s(\mu)-1} \cdot \rho\left(\frac{m\left|d_{\boldsymbol{k}}\right|}{p^{\epsilon}}\right)
$$




\section{Height PAirings on orthogonal Shimura varieties}

under the assumption $\operatorname{ord}_{p}(m) \geqslant 0$. The argument is very similar to that of [KY13, Proposition 3.1], [HY12, Proposition 2.18], [BHY15, Theorem 5.5], and [How12, Theorem 3.5.3], and hence we omit some details.

Fix one elliptic curve $\mathcal{E}$ over $\overline{\mathbb{F}}_{\mathfrak{p}}$ with complex multiplication by $\mathcal{O}_{\boldsymbol{k}}$. As the ideal class group of $\boldsymbol{k}$ acts simply transitively on the elliptic curves with complex multiplication by $\mathcal{O}_{\boldsymbol{k}}$, we find

$$
\sum_{z \in \mathcal{Z}_{0}(m, \mu)\left(\overline{\mathbb{F}}_{\mathfrak{p}}\right)} \frac{1}{\# \operatorname{Aut}(z)}=\sum_{\mathfrak{a} \in \operatorname{Pic}\left(\mathcal{O}_{\boldsymbol{k}}\right)} \sum_{\substack{x \in V_{\mu}\left(\mathcal{E} \otimes_{\mathcal{O}_{k}} \mathfrak{a}\right) \\ \operatorname{deg}(x)=m}} \frac{1}{w_{\boldsymbol{k}}} .
$$

As in the argument surrounding (4.10), the assumption Diff $L_{0}(m)=\{p\}$ implies that there is some vector

$$
x \in \operatorname{End}_{\overline{\mathcal{O}}_{k}}(\mathcal{E}) \otimes_{\mathcal{O}_{k}} V_{0}
$$

with $Q(x)=m$, and the group $\boldsymbol{k}^{1}=\left\{s \in \boldsymbol{k}^{\times}: s \bar{s}=1\right\}$ acts transitively (by right multiplication on $V_{0}$ ) on the set of all such $x$. Using $t \mapsto t / \bar{t}$ to identify $\mathbb{Q}^{\times} \backslash \boldsymbol{k}^{\times} \stackrel{\simeq}{\rightarrow} \boldsymbol{k}^{1}$ and arguing as in the proof of [HY12, Proposition 2.18], we obtain a factorization into orbital integrals

$$
\begin{aligned}
\sum_{z \in \mathcal{Z}_{0}(m, \mu)\left(\overline{\mathbb{F}}_{\mathfrak{p}}\right)} \frac{1}{\# \operatorname{Aut}(z)} & =\frac{1}{w_{\boldsymbol{k}}} \sum_{\mathfrak{a} \in \operatorname{Pic}\left(\mathcal{O}_{\boldsymbol{k}}\right)} \sum_{t \in \mathbb{Q}^{\times} \backslash \boldsymbol{k} \times} \mathbf{1}_{V_{\mu}\left(\mathcal{E} \otimes_{\mathcal{O}_{k}} \mathfrak{a}\right)}\left(x t \bar{t}^{-1}\right) \\
& =\frac{1}{2} \prod_{\ell} \sum_{t \in \mathbb{Q}_{\ell}^{\times} \backslash \boldsymbol{k}_{\ell}^{\times} / \mathcal{O}_{\boldsymbol{k}, \ell}^{\times}} \mathbf{1}_{V_{\mu}\left(E_{\ell}\right)}\left(x t \bar{t}^{-1}\right) .
\end{aligned}
$$

Here 1 indicates characteristic function, $E_{\ell}$ is the $\ell$-divisible group of $\mathcal{E}$, and

$$
V_{\mu}\left(E_{\ell}\right)=\left\{y \in \operatorname{End}_{\overline{\mathcal{O}}_{k}}\left(E_{\ell}\right) \otimes_{\mathcal{O}_{k}} L_{0}^{\vee}: y-\mathrm{id} \otimes \mu \in \operatorname{End}\left(E_{\ell}\right) \otimes_{\mathcal{O}_{k}} L_{0}\right\}
$$

On the right hand side of the first equality of (4.13) we are viewing

$$
x t \bar{t}^{-1} \in \operatorname{End}_{\overline{\mathcal{O}}_{\boldsymbol{k}}}(\mathcal{E}) \otimes_{\mathcal{O}_{\boldsymbol{k}}} V_{0} \stackrel{\simeq}{\rightarrow} \operatorname{End}_{\overline{\mathcal{O}}_{\boldsymbol{k}}}\left(\mathcal{E} \otimes_{\mathcal{O}_{\boldsymbol{k}}} \mathfrak{a}\right) \otimes_{\mathcal{O}_{k}} V_{0}
$$

using the quasi-isogeny from $\mathcal{E}$ to $\mathcal{E} \otimes \mathcal{O}_{k}$ a defined by $P \mapsto P \otimes 1$.

A choice of left $\mathcal{O}_{\boldsymbol{k}, \ell^{-}}$module isomorphism $L_{0, \ell} \stackrel{\simeq}{\rightarrow} \mathcal{O}_{\boldsymbol{k}, \ell}$ identifies

$$
V_{\mu}\left(E_{\ell}\right)=\left\{y \in \operatorname{End}_{\overline{\mathcal{O}}_{\boldsymbol{k}}}\left(E_{\ell}\right) \cdot \mathfrak{d}_{\boldsymbol{k}}^{-1}: y-\mu \in \operatorname{End}\left(E_{\ell}\right)\right\},
$$

where $\mu \in L_{0, \ell}^{\vee}=\mathfrak{d}_{\boldsymbol{k}, \ell}^{-1}$ is viewed as a quasi-endomorphism of $E_{\ell}$ using the complex multiplication. From now on we use this identification to view $x \in \operatorname{End}_{\overline{\mathcal{O}}_{\boldsymbol{k}}}\left(E_{\ell}\right) \otimes_{\mathbb{Z}_{\ell}} \mathbb{Q}_{\ell}$ and $\mu \in \boldsymbol{k}_{\ell}$. Using (4.7), the condition $m=Q(\mu)$ in $\mathbb{Q} / \mathbb{Z}$ translates to $\operatorname{Nrd}(x)=-\operatorname{Nrd}(\mu)$ in $\mathbb{Q}_{\ell} / \mathbb{Z}_{\ell}$, where $\operatorname{Nrd}$ is the reduced norm on the quaternion algebra

$$
\operatorname{End}\left(E_{\ell}\right) \otimes_{\mathbb{Z}_{\ell}} \mathbb{Q}_{\ell}=\boldsymbol{k}_{\ell} \oplus\left(\operatorname{End}_{\overline{\mathcal{O}}_{\boldsymbol{k}}}\left(E_{\ell}\right) \otimes_{\mathbb{Z}_{\ell}} \mathbb{Q}_{\ell}\right) .
$$

An explicit description (in terms of this decomposition) of the maximal order $\operatorname{End}\left(E_{\ell}\right)$ can be found in [KY13, Lemma 3.3].

Using the explicit description of $\operatorname{End}\left(E_{\ell}\right)$, the local factors on the right hand side of (4.13) can be computed directly. We demonstrate the method for a prime $\ell$ ramified in $\boldsymbol{k}$. For such an $\ell$ we have

$$
\mathbb{Q}_{\ell}^{\times} \backslash \boldsymbol{k}_{\ell}^{\times} / \mathcal{O}_{\boldsymbol{k}, \ell}^{\times}=\{1, t\}
$$


where $t \in \mathcal{O}_{\boldsymbol{k}, \ell}$ is any uniformizer. As $\ell>2$, we may choose $t$ so that $\bar{t}=-t$, and so

$$
\sum_{t \in \mathbb{Q}_{\ell}^{\times} \backslash \boldsymbol{k}_{\ell}^{\times} / \mathcal{O}_{\boldsymbol{k}, \ell}^{\times}} \mathbf{1}_{V_{\mu}\left(E_{\ell}\right)}\left(x t \bar{t}^{-1}\right)=\mathbf{1}_{V_{\mu}\left(E_{\ell}\right)}(x)+\mathbf{1}_{V_{\mu}\left(E_{\ell}\right)}(-x) .
$$

We now consider the two sub-cases $\ell=p$ and $\ell \neq p$.

If $\ell=p$, our assumption $\operatorname{ord}_{p}(m) \geqslant 0$ and the relation $m=Q(\mu)$ in $\mathbb{Q} / \mathbb{Z}$ imply that $\mu \in \mathcal{O}_{\boldsymbol{k}, p}$, and that $\operatorname{Nrd}(x) \in \mathbb{Z}_{p}$. As $\operatorname{End}\left(E_{p}\right)$ is the maximal order in the ramified quaternion algebra over $\mathbb{Q}_{p}$, this implies that $x \in \operatorname{End}\left(E_{p}\right)$. From this it follows that both $x$ and $-x$ lie in $V_{\mu}\left(E_{p}\right)$, and so

$$
\sum_{t \in \mathbb{Q}_{\ell}^{\times} \backslash \boldsymbol{k}_{\ell}^{\times} / \mathcal{O}_{\boldsymbol{k}, \ell}^{\times}} \mathbf{1}_{V_{\mu}\left(E_{\ell}\right)}\left(x t \bar{t}^{-1}\right)=2 .
$$

Now suppose $\ell \neq p$. If $\mu \in \mathcal{O}_{\boldsymbol{k}, \ell}$ then $\operatorname{Nrd}(\mu) \in \mathbb{Z}_{\ell}$, and hence $\operatorname{Nrd}(x) \in \mathbb{Z}_{\ell}$. The description of $\operatorname{End}\left(E_{\ell}\right)$ in [KY13, Lemma 3.3] now implies that $x \in \operatorname{End}\left(E_{\ell}\right)$, and again both $x$ and $-x$ lie in $V_{\mu}\left(E_{p}\right)$. If $\mu \notin \mathcal{O}_{\boldsymbol{k}, \ell}$ then $\ell \cdot \operatorname{Nrd}(\mu) \in \mathbb{Z}_{\ell}^{\times}$, and hence the relation $\operatorname{Nrd}(x)+\operatorname{Nrd}(\mu) \in \mathbb{Z}_{\ell}$ noted above implies $\ell \cdot \operatorname{Nrd}(x) \in \mathbb{Z}_{\ell}^{\times}$. Using the same relation, and the description of $\operatorname{End}\left(E_{\ell}\right)$ in $[K Y 13$, Lemma 3.3], one can show that exactly one of $x \pm \mu$ lies in $\operatorname{End}\left(E_{\ell}\right)$. Thus exactly one of $x$ and $-x$ lies in $V_{\mu}\left(E_{\ell}\right)$, and we have proved

$$
\sum_{t \in \mathbb{Q}_{\ell}^{\times} \backslash \boldsymbol{k}_{\ell}^{\times} / \mathcal{O}_{\boldsymbol{k}, \ell}^{\times}} \mathbf{1}_{V_{\mu}\left(E_{\ell}\right)}\left(x t \bar{t}^{-1}\right)= \begin{cases}1 & \text { if } \mu \notin L_{0, \ell} \\ 2 & \text { if } \mu \in L_{0, \ell} .\end{cases}
$$

From similar calculations at the unramified primes, one can deduce the relation

$$
\prod_{\ell} \sum_{t \in \mathbb{Q}_{\ell}^{\times} \backslash \boldsymbol{k}_{\ell}^{\times} / \mathcal{O}_{\boldsymbol{k}, \ell}^{\times}} \mathbf{1}_{V_{\mu}\left(E_{\ell}\right)}\left(x t \bar{t}^{-1}\right)=2^{s(\mu)} \cdot \rho\left(\frac{m\left|d_{\boldsymbol{k}}\right|}{p^{\epsilon}}\right)
$$

(the right hand side has an obvious product factorization, and one verifies equality prime-byprime.) The equality (4.12) follows immediately.

The final claim of the theorem follows by comparing the above formulas with Proposition 3.3.1.

\subsection{Mapping to the orthogonal Shimura variety}

Recall from $\S 2.3$ the GSpin Shimura variety $M$ over $\mathbb{Q}$ defined by the quadratic space $L$. In $\S 2.4$ we defined the integral model $\mathcal{M}$ over $\mathbb{Z}$, and the Kuga-Satake abelian scheme $\mathcal{A} \rightarrow \mathcal{M}$. The integral model $\mathcal{M}$ carries over it the collection of sheaves $\boldsymbol{V} \subset \underline{\operatorname{End}}(\boldsymbol{H})$ of (2.10).

The inclusion $L_{0} \rightarrow L$ extends to a homomorphism $C\left(V_{0}\right) \rightarrow C(V)$ of Clifford algebras, which then induces a morphism $\operatorname{GSpin}\left(V_{0}\right) \rightarrow \operatorname{GSpin}(V)$. The theory of canonical models shows that the induced map of complex orbifolds $Y(\mathbb{C}) \rightarrow M(\mathbb{C})$ descends to a finite and unramified morphism of $\boldsymbol{k}$-stacks

$$
Y \rightarrow M_{k}
$$

In this section we assume that the discriminant of $\boldsymbol{k}$ is odd. We will extend this morphism to a morphism $\mathcal{Y} \rightarrow \mathcal{M}_{\mathcal{O}_{k}}$ of integral models, and explain the how the various structures (special cycles, sheaves, and Kuga-Satake abelian schemes) on the source and target are related.

As noted earlier, $\mathcal{Y}$ is finite étale over $\mathcal{O}_{\boldsymbol{k}}$. The proof of Proposition 4.6.1 below will require a slightly different perspective on this, couched in the language of local models. 


\section{Height PAIRINGS ON ORThOgOnAL Shimura VARIETIES}

Let $\mathrm{M}^{\mathrm{loc}}\left(L_{0}\right)$ be the quadric over $\mathbb{Z}$ that parameterizes isotropic lines in $L_{0}$. There is an isomorphism of $\mathbb{Z}$-schemes

$$
\operatorname{Spec}\left(\mathcal{O}_{\boldsymbol{k}}\right) \stackrel{\simeq}{\rightarrow} \mathrm{M}^{\mathrm{loc}}\left(L_{0}\right)
$$

determined as follows. Consider the map $L_{0} \otimes_{\mathbb{Z}} \mathcal{O}_{\boldsymbol{k}} \rightarrow L_{0}$ defined by $v \otimes \alpha \mapsto v \bar{\alpha}$. The kernel of this map is an isotropic rank one $\mathcal{O}_{\boldsymbol{k}}$-module corresponding to a map $\operatorname{Spec}\left(\mathcal{O}_{\boldsymbol{k}}\right) \rightarrow \mathrm{M}^{\mathrm{loc}}\left(L_{0}\right)$, which is the desired isomorphism (4.15).

Now, consider the functor $\mathcal{P}_{0}$ on $\mathcal{Y}$-schemes parameterizing $\mathcal{O}_{\boldsymbol{k}}$-equivariant isomorphisms of rank two vector bundles

$$
\mathcal{O}_{\boldsymbol{k}} \otimes_{\mathbb{Z}} \mathcal{O}_{\mathcal{Y}} \stackrel{\simeq}{\rightarrow} \boldsymbol{H}_{0, \mathrm{dR}}^{+}
$$

This is represented over $\mathcal{Y}$ by a $T$-torsor $\mathcal{P}_{0} \rightarrow \mathcal{Y}$. Indeed, we only need to observe that, étale locally on $\mathcal{Y}, \boldsymbol{H}_{0, \mathrm{dR}}^{+}$is a free $\mathcal{O}_{\boldsymbol{k}} \otimes_{\mathbb{Z}} \mathcal{O}_{\mathcal{Y}}$-module of rank one.

Suppose that we are given a scheme $U \rightarrow \mathcal{Y}$ over $\mathcal{Y}$ and a section $U \rightarrow \mathcal{P}_{0}$, corresponding to

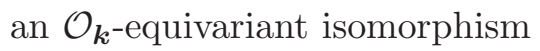

$$
\xi_{0}:\left.\mathcal{O}_{\boldsymbol{k}} \otimes_{\mathbb{Z}} \mathcal{O}_{U} \stackrel{\simeq}{\rightarrow} \boldsymbol{H}_{0, \mathrm{dR}}^{+}\right|_{U}
$$

This determines an isomorphism

$$
\left.L_{0} \otimes_{\mathbb{Z}} \mathcal{O}_{U} \stackrel{\simeq}{\rightarrow} \boldsymbol{H}_{0, \mathrm{dR}}^{+}\right|_{U} \otimes_{\mathcal{O}_{\boldsymbol{k}}} L_{0}=\left.\boldsymbol{H}_{0, \mathrm{dR}}^{-}\right|_{U} .
$$

Combining the above isomorphisms with the canonical isomorphism

$$
L_{0} \otimes_{\mathbb{Z}} \mathcal{O}_{U} \stackrel{\simeq}{\rightarrow} \underline{\operatorname{Hom}}_{\mathcal{O}_{\boldsymbol{k}}}\left(\mathcal{O}_{\boldsymbol{k}} \otimes_{\mathbb{Z}} \mathcal{O}_{U}, L_{0} \otimes_{\mathbb{Z}} \mathcal{O}_{U}\right)
$$

yields an $\mathcal{O}_{\boldsymbol{k}}$-linear isometry

$$
\xi_{0}: L_{0} \otimes_{\mathbb{Z}} \mathcal{O}_{U} \stackrel{\simeq}{\rightarrow} \underline{\operatorname{Hom}}_{\mathcal{O}_{\boldsymbol{k}}}\left(\left.\boldsymbol{H}_{0, \mathrm{dR}}^{+}\right|_{U},\left.\boldsymbol{H}_{0, \mathrm{dR}}^{-}\right|_{U}\right)=\boldsymbol{V}_{0, \mathrm{dR}}
$$

The preimage $\xi_{0}^{-1}\left(\boldsymbol{V}_{0, \mathrm{dR}}^{1}\right) \subset L_{0} \otimes \mathcal{O}_{U}$ is an isotropic line, and so corresponds to a point in $\mathrm{M}^{\mathrm{loc}}\left(L_{0}\right)(U)$. We therefore obtain a map $U \rightarrow \mathrm{M}^{\mathrm{loc}}\left(L_{0}\right)$. It is not hard to see that the composition of this map with the isomorphism (4.15) is just the structure map $U \rightarrow \operatorname{Spec}\left(\mathcal{O}_{\boldsymbol{k}}\right)$. The main point is that, as in Remark 4.3.6, $\mathcal{O}_{\boldsymbol{k}}$ acts on $\boldsymbol{V}_{0, \mathrm{dR}}^{1}$ through the structure map $\mathcal{O}_{\boldsymbol{k}} \rightarrow \mathcal{O}_{\mathcal{Y}}$, and on the quotient $\boldsymbol{V}_{0, \mathrm{dR}} / \boldsymbol{V}_{0, \mathrm{dR}}^{1}$ through the conjugate of the structure map. Hence the quotient $\boldsymbol{V}_{0, \mathrm{dR}} / \boldsymbol{V}_{0, \mathrm{dR}}^{1}$ is obtained from $\boldsymbol{V}_{0, \mathrm{dR}}$ via base-change along the augmentation map

$$
\mathcal{O}_{k} \otimes \mathcal{O}_{\mathcal{Y}} \rightarrow \mathcal{O}_{\mathcal{Y}}
$$

defined by $\alpha \otimes f \mapsto \bar{\alpha} f$.

In particular, since $\mathcal{Y}$ is étale over $\mathcal{O}_{\boldsymbol{k}}$, if $U$ is étale over $\mathcal{Y}$, then the map $U \rightarrow \mathrm{M}^{\text {loc }}\left(L_{0}\right)$ will also be étale. This can also be shown directly using Grothendieck-Messing theory.

The following result is analogous to Proposition 2.5.1.

Proposition 4.6.1. The morphism (4.14) extends uniquely to a finite and unramified morphism

$$
\mathcal{Y} \rightarrow \mathcal{M}_{\mathcal{O}_{k}}
$$

and there is a canonical $C(L)$-equivariant isomorphism

$$
\mathcal{A}_{0}\left|\mathcal{Y} \otimes_{C\left(L_{0}\right)} C(L) \stackrel{\simeq}{\rightarrow} \mathcal{A}\right|_{\mathcal{Y}}
$$

of $\mathbb{Z} / 2 \mathbb{Z}$-graded abelian schemes over $\mathcal{Y}$. In particular,

$$
\boldsymbol{H}_{0}\left|\mathcal{Y} \otimes_{C\left(L_{0}\right)} C(L) \stackrel{\simeq}{\rightarrow} \boldsymbol{H}\right|_{\mathcal{Y}}
$$


Furthermore, set $\boldsymbol{\Lambda}=\Lambda \otimes \mathbf{1}$, and recall the space of special endomorphisms

$$
V\left(\left.\mathcal{A}\right|_{\mathcal{Y}}\right) \subset \operatorname{End}_{C(L)}\left(\left.\mathcal{A}\right|_{\mathcal{Y}}\right)
$$

of $\S 2.6$. There is a canonical embedding $\Lambda \subset V\left(\left.\mathcal{A}\right|_{\mathcal{Y}}\right)$ with the following properties:

(i) Its homological realization exhibits $\left.\boldsymbol{\Lambda} \subset \boldsymbol{V}\right|_{\mathcal{Y}}$ as a local direct summand.

(ii) The injection

$$
\underline{\operatorname{End}}_{C\left(L_{0}\right)}\left(\boldsymbol{H}_{0} \mid \mathcal{Y}\right) \rightarrow \underline{\operatorname{End}}_{C(L)}(\boldsymbol{H} \mid \mathcal{Y})
$$

induced by (4.18) identifies $\left.\boldsymbol{V}_{0}\right|_{\mathcal{Y}}$ with the submodule of all elements of $\left.\boldsymbol{V}\right|_{\mathcal{Y}}$ anticommuting with all elements of $\boldsymbol{\Lambda}$. Furthermore $\left.\left.\boldsymbol{V}_{0}\right|_{\mathcal{Y}} \subset \boldsymbol{V}\right|_{\mathcal{Y}}$ is locally a direct summand.

(iii) In the de Rham case, the inclusion $\boldsymbol{V}_{0, \mathrm{dR}}\left|\mathcal{Y} \subset \boldsymbol{V}_{\mathrm{dR}}\right| \mathcal{Y}$ identifies $\boldsymbol{V}_{0, \mathrm{dR}}^{1}\left|\mathcal{Y}=\boldsymbol{V}_{\mathrm{dR}}^{1}\right| \mathcal{Y}$.

Proof. Just as in (2.11), there is a $C(L)$-equivariant isomorphism

$$
\left.\left.\mathcal{A}_{0}\right|_{Y} \otimes_{C\left(L_{0}\right)} C(L) \stackrel{\simeq}{\rightarrow} \mathcal{A}\right|_{Y}
$$

of $\mathbb{Z} / 2 \mathbb{Z}$-graded abelian schemes over $Y$.

Extending the map of (4.14) of $\boldsymbol{k}$-stacks to a map (4.16) of $\mathcal{O}_{\boldsymbol{k}}$-stacks is equivalent to extending the map of $\mathbb{Q}$-stacks $Y \rightarrow M$ to a map of $\mathbb{Z}$-stacks $\mathcal{Y}_{\mathbb{Z}} \rightarrow \mathcal{M}$. Here we write $\mathcal{Y}_{\mathbb{Z}}$ for the stack $\mathcal{Y}$ viewed over $\operatorname{Spec}(\mathbb{Z})$.

To do this, fix a prime $p>0$, and assume first that $L_{\mathbb{Z}_{(p)}}$ is self-dual. As observed in $\S 2.4$, an appropriate choice of $\delta \in C^{+}(L) \cap C(V)^{\times}$determines a degree $d$ polarization of the Kuga-Satake abelian scheme $\mathcal{A}_{M} \rightarrow M$, inducing a finite unramified map

$$
i_{\delta}: M \rightarrow \mathcal{X}_{2^{n+1}, d, \mathbb{Q}}^{\text {Siegel }}
$$

to a moduli stack of polarized abelian varieties. The integral model $\mathcal{M}_{\mathbb{Z}_{(p)}}$ is then simply the normalization of $\mathcal{X}_{2^{n+1}, d, \mathbb{Z}_{(p)}}^{\text {Siegel }}$ in $M$.

The composition

$$
Y \rightarrow M \rightarrow \mathcal{X}_{2^{n+1}, d, \mathbb{Q}}^{\text {Siegel }}
$$

determines a polarized abelian scheme over $Y$, which is precisely (4.19). The polarized abelian scheme $\left.\mathcal{A}_{0}\right|_{\mathcal{Y}_{(p)}} \otimes_{C\left(L_{0}\right)} C(L)$ defines an extension of (4.19) to $\mathcal{Y}_{\mathbb{Z}_{(p)}}$, which shows that the above composition extends to a morphism

$$
\mathcal{Y}_{\mathbb{Z}_{(p)}} \rightarrow \mathcal{X}_{2^{n+1}, d, \mathbb{Z}_{(p)}}^{\text {Siegel }}
$$

Since $\mathcal{Y}$ is regular, and hence normal, this extension must lift to a map $\mathcal{Y}_{\mathbb{Z}_{(p)}} \rightarrow \mathcal{M}_{\mathbb{Z}_{(p)}}$, and by construction (4.19) extends to an isomorphism

$$
\left.\left.\left(\mathcal{A}_{0} \otimes_{C\left(L_{0}\right)} C(L)\right)\right|_{\mathcal{Y}_{(p)}} \stackrel{\simeq}{\rightarrow} \mathcal{A}\right|_{\mathcal{Y}_{(p)}} .
$$

The construction of the embedding $\Lambda \hookrightarrow\left(\left.\mathcal{A}\right|_{\mathcal{Y}_{\mathbb{Z}_{(p)}}}\right)$, as well as the proofs of assertions (i), (ii) and (iii) over $\mathcal{Y}_{\mathbb{Z}_{(p)}}$, now proceed exactly as in Proposition 2.5.1.

It remains to show (under our self-duality assumption on $L_{\mathbb{Z}_{(p)}}$ ) that the map $\mathcal{Y}_{\mathbb{Z}_{(p)}} \rightarrow \mathcal{M}_{\mathbb{Z}_{(p)}}$ is finite and unramified. For this, using Proposition 2.4.3 and the above discussion of local models, we see that the map is étale locally on the source isomorphic to an étale neighborhood of the closed immersion $\mathrm{M}^{\mathrm{loc}}\left(L_{0}\right) \rightarrow \mathrm{M}^{\mathrm{loc}}(L)$. This shows that $\mathcal{Y}_{\mathbb{Z}_{(p)}}$ is unramified over $\mathcal{M}_{\mathbb{Z}_{(p)}}$. That it is also finite is immediate from the fact that $\mathcal{Y}$ is finite over $\mathcal{O}_{\boldsymbol{k}}$. 


\section{Height PAIRINGs on ORTHOgOnAL Shimura VARIETIES}

It remains to deal with the primes $p$ where $L_{\mathbb{Z}_{(p)}}$ is not self-dual. For this, embed $L$ as an isometric direct summand of a maximal lattice $L^{\diamond}$ of signature $\left(n^{\diamond}, 2\right)$ for which $L_{\mathbb{Z}_{(p)}}$ is self-dual. Let $\mathcal{M}^{\diamond}$ be the regular integral model over $\mathbb{Z}$ for the Shimura variety $M^{\diamond}$ associated with $L^{\diamond}$. As in (2.4), let $\check{\mathcal{M}}_{\mathbb{Z}_{(p)}}$ be the normalization of $\mathcal{M}_{\mathbb{Z}_{(p)}}^{\diamond}$ in $M$.

From what we have shown above, the composition $Y \rightarrow M \rightarrow M^{\diamond}$ extends to a map $\mathcal{Y}_{\mathbb{Z}_{(p)}} \rightarrow$ $\mathcal{M}_{\mathbb{Z}_{(p)}}^{\diamond}$, which, since $\mathcal{Y}$ is a normal stack over $\mathbb{Z}$, must lift to a finite map $\mathcal{Y}_{\mathbb{Z}_{(p)}} \rightarrow \check{\mathcal{M}}_{\mathbb{Z}_{(p)}}$. As in the proof of Proposition 2.5.1, we find that that this lift must map $\mathcal{Y}_{\mathbb{Z}_{(p)}}$ to $\mathcal{M}_{\mathbb{Z}_{(p)}}$ and must also be unramified, being, étale locally on the source, isomorphic to an étale neighborhood of

$$
\mathrm{M}^{\mathrm{loc}}\left(L_{0}\right)_{\mathbb{Z}} \rightarrow \mathrm{M}^{\mathrm{loc}}(L)
$$

If $p=2$ the assumption that $C^{+}\left(L_{0}\right)$ is a maximal order of odd discriminant implies that $L_{0, \mathbb{F}_{2}}$ is self-dual so that (4.20) must map into the smooth locus of the target.

For $p>2$ the key point is that the radical of $L_{0, \mathbb{F}_{p}}$, if non-zero, defines an $\mathbb{F}_{p}$-valued point of the quadric $\mathrm{M}^{\text {loc }}\left(L_{0}\right)$ associated with $L_{0}$. Therefore (4.20) must map into the regular locus (see the proof of Proposition 2.5.1).

Finally, the extension (4.17) of the isomorphism (4.19) once again follows from the property of Néron models, and the remaining assertions follow from Proposition 2.5.1 and from what we have already shown in the self-dual case.

Remark 4.6.2. The proof of Proposition 4.6.1 actually proves the stronger claim that the morphism $\mathcal{Y} \rightarrow \mathcal{M}$ is finite and unramified.

Now suppose that $S$ is a scheme over $\mathcal{Y}$. Then Proposition 4.6.1 gives us an isometric embed$\operatorname{ding} \Lambda \hookrightarrow V\left(\mathcal{A}_{S}\right)$. We obtain the following analogue of Proposition 2.6.4.

Proposition 4.6.3.

(i) There is a canonical isometry

$$
V\left(\mathcal{E}_{S}\right) \stackrel{\simeq}{\rightarrow} \Lambda^{\perp} \subset V\left(\mathcal{A}_{S}\right) .
$$

(ii) The induced map $V\left(\mathcal{E}_{S}\right) \oplus \Lambda \hookrightarrow V\left(\mathcal{A}_{S}\right)$, tensored with $\mathbb{Q}$, restricts to an injection

$$
V_{\mu_{1}}\left(\mathcal{E}_{S}\right) \times\left(\mu_{2}+\Lambda\right) \hookrightarrow V_{\mu}\left(\mathcal{A}_{S}\right)
$$

for every $\mu \in L^{\vee} / L$ and every

$$
\left(\mu_{1}, \mu_{2}\right) \in(\mu+L) /\left(L_{0} \oplus \Lambda\right) \subset\left(L_{0}^{\vee} / L_{0}\right) \oplus\left(\Lambda^{\vee} / \Lambda\right) .
$$

(iii) The above injections determine a decomposition

$$
V_{\mu}\left(\mathcal{A}_{S}\right)=\bigsqcup_{\left(\mu_{1}, \mu_{2}\right) \in(\mu+L) /\left(L_{0} \oplus \Lambda\right)} V_{\mu_{1}}\left(\mathcal{E}_{S}\right) \times\left(\mu_{2}+\Lambda\right) .
$$

Proof. Exactly as in the proof Proposition 2.6.4, Proposition 4.6.1 implies that there is a canonical isometry

$$
V\left(\mathcal{A}_{0, S}\right) \stackrel{\simeq}{\rightarrow} \Lambda^{\perp} \subset V\left(\mathcal{A}_{S}\right) .
$$

Thus assertion (i) is clear from (4.8).

In particular, there is a canonical inclusion $V\left(\mathcal{E}_{S}\right) \times \Lambda \subset V\left(\mathcal{A}_{S}\right)$, which we can make explicit using the $C(L)$-equivariant isomorphism $\mathcal{E}_{S} \otimes \mathcal{O}_{k} C(L) \stackrel{\simeq}{\rightarrow} \mathcal{A}_{S}$ of (4.17). Indeed, each $\lambda \in \Lambda$ induces a special endomorphism

$$
\mathcal{A}_{S} \stackrel{\simeq}{\rightarrow} \mathcal{E}_{S} \otimes_{\mathcal{O}_{k}} C(L) \stackrel{e \otimes c \mapsto e \otimes \lambda c}{\longrightarrow} \mathcal{E}_{S} \otimes_{\mathcal{O}_{k}} C(L) \stackrel{\simeq}{\rightarrow} \mathcal{A}_{S}
$$


while each $\phi \otimes \ell \in \operatorname{End}_{\overline{\mathcal{O}}_{\boldsymbol{k}}}\left(\mathcal{E}_{S}\right) \otimes \mathcal{O}_{\boldsymbol{k}} L_{0}=V\left(\mathcal{E}_{S}\right)$ induces a special endomorphism

$$
\mathcal{A}_{S} \stackrel{\simeq}{\rightarrow} \mathcal{E}_{S} \otimes \mathcal{O}_{k} C(L) \stackrel{e \otimes c \mapsto \phi(e) \otimes \ell c}{\longrightarrow} \mathcal{E}_{S} \otimes \mathcal{O}_{k} C(L) \stackrel{\simeq}{\longrightarrow} \mathcal{A}_{S} .
$$

With this in mind, assertions (ii) and (iii) amount to unwinding the definitions. By embedding $L$ into a unimodular lattice and using Proposition 2.6.4, we may reduce to the case where $L$ is itself unimodular. In this case, we have canonical isometries

$$
L_{0}^{\vee} / L_{0} \stackrel{\simeq}{\longleftarrow} L /\left(L_{0} \oplus \Lambda\right) \stackrel{\simeq}{\rightarrow} \Lambda^{\vee} / \Lambda
$$

and $\mu_{1} \in L_{0}^{\vee} / L_{0}$ corresponds to $\mu_{2} \in \Lambda^{\vee} / \Lambda$ if and only if $\left(\mu_{1}, \mu_{2}\right) \in L /\left(L_{0} \oplus \Lambda\right)$.

If $\left(\mu_{1}, \mu_{2}\right) \in L /\left(L_{0} \oplus \Lambda\right)$ and

$$
(x, \lambda) \in V_{\mu_{1}}\left(\mathcal{E}_{S}\right) \times\left(\mu_{2}+\Lambda\right)
$$

then we may write $x=\phi \otimes \ell+\mathrm{id} \otimes \mu_{1}$ for some $\phi \in \operatorname{End}_{\overline{\mathcal{O}}_{\boldsymbol{k}}}\left(\mathcal{E}_{S}\right)$ and $\ell \in L_{0}$. As $\mu_{1}+\mu_{2} \in L$, the quasi-endomorphism $(x, \lambda) \in V\left(\mathcal{A}_{S}\right)_{\mathbb{Q}}$ is equal to

$$
\mathcal{A}_{S} \stackrel{\simeq}{\rightarrow} \mathcal{E}_{S} \otimes_{\mathcal{O}_{k}} C(L) \stackrel{e \otimes c \mapsto \phi(e) \otimes \ell c+e \otimes\left(\mu_{1}+\mu_{2}\right) c}{\longrightarrow} \mathcal{E}_{S} \otimes_{\mathcal{O}_{k}} C(L) \stackrel{\simeq}{\rightarrow} \mathcal{A}_{S},
$$

and so lies in $V\left(\mathcal{A}_{S}\right)$.

Conversely, any element of $V\left(\mathcal{A}_{S}\right)$ is given by a pair

$$
(x, \lambda) \in V\left(\mathcal{E}_{S}\right)_{\mathbb{Q}} \times \Lambda_{\mathbb{Q}},
$$

and it is easy to see that we must have $x \in V\left(\mathcal{E}_{S}\right)^{\vee}$ and $\lambda \in \Lambda^{\vee}$. Let $\mu_{2} \in \Lambda^{\vee} / \Lambda$ be the coset of $\lambda$, and let $\mu_{1} \in L_{0}^{\vee} / L_{0}$ be the unique element for which $\left(\mu_{1}, \mu_{2}\right) \in L /\left(L_{0} \oplus \Lambda\right)$. If we write $x=\phi \otimes \ell$ with $\ell \in V_{0}$ then, by assumption, the quasi-endomorphism of $\mathcal{A}_{S}$ determined by $(x, \lambda)$ is the (integral) endomorphism (4.21), while $x-\mathrm{id} \otimes \mu_{1}$ is the quasi-endomorphism

$$
\mathcal{A}_{S} \stackrel{\simeq}{\rightarrow} \mathcal{E}_{S} \otimes_{\mathcal{O}_{k}} C(L) \stackrel{e \otimes c \mapsto \phi(e) \otimes \ell c-e \otimes \mu_{1} c}{\longrightarrow} \mathcal{E}_{S} \otimes_{\mathcal{O}_{k}} C(L) \stackrel{\simeq}{\rightarrow} \mathcal{A}_{S}
$$

These differ by the endomorphism $e \otimes c \mapsto e \otimes\left(\mu_{1}+\mu_{2}\right) c$, and so $x-\mathrm{id} \otimes \mu_{1}$ is an (integral) endomorphism of $\mathcal{E}_{S} \otimes_{\mathcal{O}_{k}} C(L)$. As $L_{0} \subset C(L)$ as an $\mathcal{O}_{\boldsymbol{k}}$-module direct summand, it follows that $x-\mathrm{id} \otimes \mu_{1}$ restricts to a homomorphism $\mathcal{E}_{S} \rightarrow \mathcal{E}_{S} \otimes \mathcal{O}_{k} L_{0}$. This proves that $x \in V_{\mu_{1}}\left(\mathcal{E}_{S}\right)$, and all parts of the claim follow.

We immediately obtain from Proposition 4.6.3 the following analogue of Proposition 2.7.1. Proposition 4.6.4. There is an isomorphism of $\mathcal{Y}$-stacks

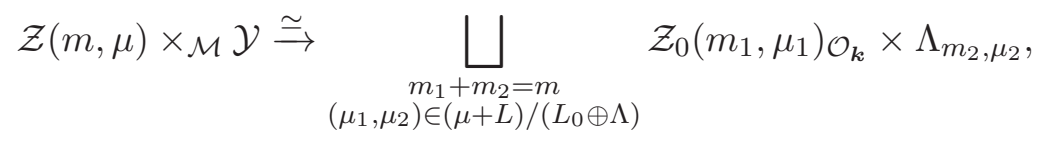

where

$$
\Lambda_{m_{2}, \mu_{2}}=\left\{x \in \mu_{2}+\Lambda: Q(x)=m_{2}\right\},
$$

and $\mathcal{Z}_{0}\left(m_{1}, \mu_{1}\right)_{\mathcal{O}_{k}} \times \Lambda_{m_{2}, \mu_{2}}$ denotes the disjoint union of $\# \Lambda_{m_{2}, \mu_{2}}$ copies of $\mathcal{Z}_{0}\left(m_{1}, \mu_{1}\right)_{\mathcal{O}_{k}}$.

Remark 4.6.5. The terms $\mathcal{Z}_{0}\left(m_{1}, \mu_{1}\right)_{\mathcal{O}_{k}}$ in the decomposition (4.22) define 0-cycles on $\mathcal{Y}$ when $m_{1} \neq 0$. The remaining terms

$$
\bigsqcup_{\left(0, \mu_{2}\right) \in(\mu+L) /\left(L_{0} \oplus \Lambda\right)} \mathcal{Z}_{0}(0,0)_{\mathcal{O}_{k}} \times \Lambda_{m, \mu_{2}}=\bigsqcup_{\left(0, \mu_{2}\right) \in(\mu+L) /\left(L_{0} \oplus \Lambda\right)} \mathcal{Y} \times \Lambda_{m, \mu_{2}}
$$

are those with $\left(m_{1}, \mu_{1}\right)=(0,0)$, and account for the improper intersection between the cycles $\mathcal{Z}(m, \mu)_{\mathcal{O}_{k}}$ and $\mathcal{Y}$. 


\section{Height PAIRINGS ON ORTHOGONAL Shimura VARIETIES}

\section{Degrees of metrized line bundles}

As in previous sections, fix a maximal quadratic space $L$ over $\mathbb{Z}$ of signature $(n, 2)$, and a $\mathbb{Z}$ module direct summand $L_{0} \subset L$ of signature $(0,2)$. Assume the even Clifford algebra $C^{+}\left(L_{0}\right)$ is isomorphic to the maximal order in a quadratic imaginary field $\boldsymbol{k} \subset \mathbb{C}$ of odd discriminant. Set

$$
\Lambda=\left\{x \in L: x \perp L_{0}\right\} .
$$

Recall from $\S 2.4$ that we have associated with $L$ an algebraic stack

$$
\mathcal{M} \rightarrow \operatorname{Spec}\left(\mathcal{O}_{\boldsymbol{k}}\right) .
$$

Similarly, in $\S 4.3$ we constructed from $L_{0}$ an algebraic stack $\mathcal{Y} \rightarrow \operatorname{Spec}\left(\mathcal{O}_{\boldsymbol{k}}\right)$. The functoriality results of Proposition 4.6.1 provide us with a finite and unramified morphism

$$
i: \mathcal{Y} \rightarrow \mathcal{M} \text {. }
$$

Of course the stack $\mathcal{M}$ was originally constructed over $\mathbb{Z}$. Its restriction to $\mathcal{O}_{\boldsymbol{k}}$ is all that we will use in this section.

\subsection{Metrized line bundles}

We will need some basic notions of arithmetic intersection theory on the stack $\mathcal{M}$. For more details see [GS90], [KRY04], [KRY06], or [Vis89]

Although the integral model over $\mathbb{Z}$ described in $\S 2.4$ is regular, its base change (5.1) to $\mathcal{O}_{\boldsymbol{k}}$ need not be. This is not a serious problem: the first part of the following proposition implies that $\mathcal{M}$ has a perfectly good theory of Cartier divisors. Whenever we speak of "divisors" on $\mathcal{M}$ we always mean "Cartier divisors."

Proposition 5.1.1. The stack $\mathcal{M}$ is locally integral. If $D$ denotes the greatest common divisor of $\operatorname{disc}\left(L_{0}\right)$ and $\operatorname{disc}(L)$, then the restriction of $\mathcal{M}$ to $\mathcal{O}_{\boldsymbol{k}}[1 / D]$ is regular.

Proof. Using Proposition 2.4.3 and the definition of $\mathcal{M}$, to show that $\mathcal{M}$ is locally integral, it suffices to show that the regular locus of the quadric $\mathrm{M}^{\mathrm{loc}}(L)$ associated with the quadratic space $L$ is integral after base change to $\mathcal{O}_{\boldsymbol{k}}$. In explicit terms, the complete local rings of this locus at its $\overline{\mathbb{F}}_{p}$-points are either formally smooth over $\mathbb{Z}_{p}$, or are isomorphic to one of the two following $W\left(\overline{\mathbb{F}}_{p}\right)$-algebras [MP16, (2.16)]:

$$
W\left(\overline{\mathbb{F}}_{p}\right) \frac{\left[\left[u_{1}, \ldots, u_{n+1}\right]\right]}{\left(\sum_{i} u_{i}^{2}+p\right)} \text {, or } \quad W\left(\overline{\mathbb{F}}_{p}\right) \frac{\left[\left[u_{1}, \ldots, u_{n}, w\right]\right]}{\left(\sum_{i} u_{i}^{2}+p(w+1)\right)} .
$$

Since $n \geqslant 1$, it is easily checked that, in all three cases, tensoring with $\mathcal{O}_{\boldsymbol{k}}$ over $\mathbb{Z}$ gives us something locally integral.

The claim about regularity over $\mathcal{O}_{\boldsymbol{k}}[1 / D]$ follows from the smoothness of the integral model of $\S 2.4$ over $\mathbb{Z}[1 / \operatorname{disc}(L)]$ and of $\mathcal{O}_{\boldsymbol{k}}\left[1 / \operatorname{disc}\left(L_{0}\right)\right]$ over $\mathbb{Z}$.

An arithmetic divisor on $\mathcal{M}$ is a pair $\widehat{\mathcal{Z}}=(\mathcal{Z}, \Phi)$ consisting of a Cartier divisor $\mathcal{Z}$ on $\mathcal{M}$ and a Green function $\Phi$ for $\mathcal{Z}(\mathbb{C})$ on $\mathcal{M}(\mathbb{C})$. Thus if $\Psi=0$ is a local equation for $\mathcal{Z}(\mathbb{C})$, the function $\Phi+\log |\Psi|^{2}$, initially defined on the complement of $\mathcal{Z}(\mathbb{C})$, is required to extend smoothly across the singularity $\mathcal{Z}(\mathbb{C})$. A principal arithmetic divisor is an arithmetic divisor of the form

$$
\widehat{\operatorname{div}}(\Psi)=\left(\operatorname{div}(\Psi),-\log |\Psi|^{2}\right)
$$




\section{F. Andreatta, E. Z. Goren, B. Howard, K. Madapusi Pera}

for a rational function $\Psi$ on $\mathcal{M}$. The group of all arithmetic divisors is denoted $\widehat{\operatorname{Div}}(\mathcal{M})$, and its quotient by the subgroup of principal arithmetic divisors is the arithmetic Chow group $\widehat{\mathrm{CH}}^{1}(\mathcal{M})$ of Gillet-Soulé.

A metrized line bundle on $\mathcal{M}$ is a line bundle endowed with a smoothly varying Hermitian metric on its complex points. The isomorphism classes of metrized line bundles form a group $\widehat{\operatorname{Pic}}(\mathcal{M})$ under tensor product.

There is an isomorphism

$$
\widehat{\mathrm{CH}}^{1}(\mathcal{M}) \stackrel{\simeq}{\rightarrow} \widehat{\operatorname{Pic}}(\mathcal{M})
$$

defined as follows. Given an arithmetic divisor $(\mathcal{Z}, \Phi)$, the line bundle $\mathcal{L}=\mathcal{O}(\mathcal{Z})$ is endowed with a canonical rational section $s$ with divisor $\mathcal{Z}$. This section is nothing more than the constant function 1 on $\mathcal{M}$, viewed as a section of $\mathcal{L}$. We endow $\mathcal{L}$ with the metric defined by $-\log \|s\|^{2}=\Phi$. For the inverse construction, start with a metrized line bundle $\widehat{\mathcal{L}}$ on $\mathcal{M}$ and let $s$ be any nonzero rational section. The associated arithmetic divisor, well-defined modulo principal arithmetic divisors, is

$$
\widehat{\operatorname{div}}(s)=\left(\operatorname{div}(s),-\log \|s\|^{2}\right) .
$$

Of course there is a similar discussion with $\mathcal{M}$ replaced by $\mathcal{Y}$. As $\mathcal{Y}$ is smooth of relative dimension 0 over $\mathcal{O}_{\boldsymbol{k}}$, all divisors on $\mathcal{Y}$ are supported in nonzero characteristics; thus, a Green function for a divisor on $\mathcal{Y}$ is any complex-valued function on the 0 -dimensional orbifold $\mathcal{Y}(\mathbb{C})$. In particular any arithmetic divisor $(\mathcal{Z}, \Phi)$ decomposes as $(\mathcal{Z}, 0)+(0, \Phi)$, and $\mathcal{Z}$ can be further decomposed as the difference of two effective Cartier divisors.

To define the arithmetic degree (as in [KRY04] or [KRY06]) of an arithmetic divisor $\widehat{\mathcal{Z}}$ on $\mathcal{Y}$ we first assume that $\widehat{\mathcal{Z}}=(\mathcal{Z}, 0)$ with $\mathcal{Z}$ effective. Then

$$
\widehat{\operatorname{deg}}(\widehat{\mathcal{Z}})=\sum_{\mathfrak{p} \subset \mathcal{O}_{\boldsymbol{k}}} \log \mathrm{N}(\mathfrak{p}) \sum_{z \in \mathcal{Z}\left(\overline{\mathbb{F}}_{\mathfrak{p}}\right)} \frac{\operatorname{length}\left(\mathcal{O}_{\mathcal{Z}, z}\right)}{\# \operatorname{Aut}(z)}
$$

where $\mathcal{O}_{\mathcal{Z}, z}$ is the étale local ring of $\mathcal{Z}$ at $z$. If $\widehat{\mathcal{Z}}=(0, \Phi)$ is purely archimedean, then

$$
\widehat{\operatorname{deg}}(\widehat{\mathcal{Z}})=\sum_{y \in \mathcal{Y}(\mathbb{C})} \frac{\Phi(y)}{\# \operatorname{Aut}(y)} .
$$

The arithmetic degree extends linearly to all arithmetic divisors, and defines a homomorphism

$$
\widehat{\operatorname{deg}}: \widehat{\operatorname{Pic}}(\mathcal{Y}) \rightarrow \mathbb{R} \text {. }
$$

We now define a homomorphism

$$
[\cdot: \mathcal{Y}]: \widehat{\operatorname{Pic}}(\mathcal{M}) \rightarrow \mathbb{R},
$$

the arithmetic degree along $\mathcal{Y}$, as the composition

$$
\widehat{\operatorname{Pic}}(\mathcal{M}) \stackrel{i^{*}}{\rightarrow} \widehat{\operatorname{Pic}}(\mathcal{Y}) \stackrel{\widehat{\operatorname{deg}}}{\longrightarrow} \mathbb{R} .
$$

\subsection{Specialization to the normal bundle}

Fix a positive $m \in \mathbb{Q}$ and a $\mu \in L^{\vee} / L$, and denote by

$$
\mathcal{Z}(m, \mu) \rightarrow \mathcal{M}
$$

the stack obtained from (2.21) by base change from $\mathbb{Z}$ to $\mathcal{O}_{\boldsymbol{k}}$. The fact that (5.2) and $i: \mathcal{Y} \rightarrow \mathcal{M}$ are not closed immersions is a minor nuisance. The following definition is introduced to address 


\section{Height PAIRINGs on ORTHOgOnal Shimura varieties}

technical difficulties arising from this defect.

Definition 5.2.1. A sufficiently small étale open chart of $\mathcal{M}$ is a scheme $U$ together with an étale morphism $U \rightarrow \mathcal{M}$ such that

(i) the natural map $\mathcal{Z}(m, \mu)_{U} \rightarrow U$ restricts to a closed immersion on every connected component $Z \subset \mathcal{Z}(m, \mu)_{U}$

(ii) the natural map $\mathcal{Y}_{U} \rightarrow U$ restricts to a closed immersion on every connected component $Y \subset \mathcal{Y}_{U}$

Remark 5.2.2. The stack $\mathcal{M}$ admits a covering by sufficiently small étale open charts. This is a consequence of [Vis89, Lemma 1.19] and the fact that $\mathcal{Z}(m, \mu) \rightarrow \mathcal{M}$ and $\mathcal{Y} \rightarrow \mathcal{M}$ are finite and unramified. Note that these two morphisms are relatively representable, and so for any $U \rightarrow \mathcal{M}$ as above the pull-backs $\mathcal{Z}(m, \mu)_{U}$ and $\mathcal{Y}_{U}$ are actually schemes.

Remark 5.2.3. When we think of $\mathcal{Z}(m, \mu)$ as a Cartier divisor on $\mathcal{M}$, its pull-back to $U$ is simply the sum of the connected components of $\mathcal{Z}(m, \mu)_{U}$, each viewed as closed subscheme of $U$.

Remark 5.2.4. In $\S 4$ the notation $Y$ was used for the generic fiber of $\mathcal{Y}$. Throughout $\S 5$ the notation $Y$ is only used for a connected component of $\mathcal{Y}_{U}$, and so no confusion should arise.

Fix a sufficiently small $U \rightarrow \mathcal{M}$ and a connected component $Y \subset \mathcal{Y}_{U}$. The smoothness of $\mathcal{Y}$ over $\mathcal{O}_{k}$ implies that $Y$ is a regular integral scheme of dimension one. Let $I \subset \mathcal{O}_{U}$ be the ideal sheaf defining the closed immersion $Y \rightarrow U$, and recall that the normal bundle of $Y \subset U$ is the $\mathcal{O}_{Y}$-module

$$
N_{Y} U=\underline{\operatorname{Hom}}\left(I / I^{2}, \mathcal{O}_{Y}\right) .
$$

As $Y \rightarrow U$ may not be a regular immersion, the $\mathcal{O}_{Y}$-module $I / I^{2}$ may not be locally free. However, we will see below in Proposition 5.4.1 that $N_{Y} U$ is locally free of rank $n$, and so defines a vector bundle $N_{Y} U \rightarrow Y$. By taking the disjoint union over all connected components $Y \subset \mathcal{Y}_{U}$ we obtain a vector bundle on $\mathcal{Y}_{U}$. Letting $U$ vary over a cover of $\mathcal{M}$ by sufficiently small étale open charts and glueing defines the normal bundle

$$
\pi: N_{\mathcal{Y}} \mathcal{M} \rightarrow \mathcal{Y}
$$

Recall the Hejhal-Poincaré series

$$
F_{m, \mu}(\tau) \in H_{1-\frac{n}{2}}\left(\omega_{L}\right)
$$

of $\S 3.2$. The discussion of $\S 3.2$ endows the divisor $\mathcal{Z}(m, \mu)=\mathcal{Z}\left(F_{m, \mu}\right)$ with a Green function

$$
\Phi_{m, \mu}=\Phi\left(F_{m, \mu}, \cdot\right),
$$

which is defined at every point of $\mathcal{M}(\mathbb{C})$, but is discontinuous at points of the divisor $\mathcal{Z}(m, \mu)$. The corresponding arithmetic divisor is denoted

$$
\widehat{\mathcal{Z}}(m, \mu)=\left(\mathcal{Z}(m, \mu), \Phi_{m, \mu}\right) \in \widehat{\operatorname{Div}}(\mathcal{M}) .
$$

From the arithmetic divisor (5.4) we will construct a new arithmetic divisor

$$
\sigma(\widehat{\mathcal{Z}}(m, \mu)) \in \widehat{\operatorname{Div}}\left(N_{\mathcal{Y}} \mathcal{M}\right)
$$

called the specialization to the normal bundle. Fix a cover of $\mathcal{M}$ by sufficiently small étale open charts. Given a chart $U \rightarrow \mathcal{M}$ in the cover, fix a connected component $Y \subset \mathcal{Y}_{U}$ and decompose $\mathcal{Z}(m, \mu)_{U}=\bigsqcup Z$ as the union of its connected components. By refining our cover, we may assume that each closed subscheme $Z \rightarrow U$ is defined by a single equation $f_{Z}=0$. 
Lemma 5.2.5. For every connected component $Z \subset \mathcal{Z}(m, \mu)_{U}$ the intersection

$$
Y \cap Z:=Y \times_{U} Z
$$

satisfies one of the following (mutually exclusive) properties:

(i) $Y \cap Z$ has dimension 0 , and $f_{Z}$ restricts to a nonzero section of $\mathcal{O}_{U} / I$,

(ii) $Y \cap Z=Y$, and $f_{Z}$ defines a section of $I \subset \mathcal{O}_{U}$ with nonzero image under the natural map $I / I^{2} \rightarrow \underline{\operatorname{Hom}}\left(N_{U} Y, \mathcal{O}_{Y}\right)$.

Proof. As $Y$ is an integral scheme of dimension one, its closed subscheme $Y \cap Z$ is either all of $Y$ or of dimension 0. Given this, the only nontrivial thing to check is that when $Y \cap Z=Y$, the image of $f_{Z}$ under $I / I^{2} \rightarrow \underline{\operatorname{Hom}}\left(N_{U} Y, \mathcal{O}_{Y}\right)$ is nonzero. This can be checked after restricting to the complex fiber, where it follows from the smoothness of the divisor $Z(\mathbb{C})$ on $U(\mathbb{C})$. This smoothness can be checked using the complex uniformization (2.4).

If $Y \cap Z$ has dimension 0 , then restricting $f_{Z}$ to $Y$ and pulling back via $N_{Y} U \rightarrow Y$ results in a function $\sigma\left(f_{Z}\right)$ on $N_{Y} U$, which is homogeneous of degree zero. On the other hand, if $Y \cap Z=Y$ then the image of $f_{Z}$ in $\underline{\operatorname{Hom}}\left(N_{U} Y, \mathcal{O}_{Y}\right)$ defines a function $\sigma\left(f_{Z}\right)$ on $N_{U} Y$, which is homogeneous of degree one. In either case, define an effective Cartier divisor on $N_{Y} U$ by

$$
\sigma(\mathcal{Z}(m, \mu))=\sum_{Z \subset \mathcal{Z}(m, \mu)_{U}} \operatorname{div}\left(\sigma\left(f_{Z}\right)\right) .
$$

The function

$$
\phi_{m, \mu}=\Phi_{m, \mu}+\sum_{Z \subset \mathcal{Z}(m, \mu)_{U}} \log \left|f_{Z}\right|^{2}
$$

initially defined on the complex fiber of $U \backslash \mathcal{Z}(m, \mu)_{U}$, extends smoothly to all of $U(\mathbb{C})$. By restricting to $\mathcal{Y}(\mathbb{C})$ and then pulling back via (5.3) we obtain a smooth function $\pi^{*} i^{*} \phi_{m, \mu}$ on $\left(N_{Y} U\right)(\mathbb{C})$. Define $\sigma\left(\Phi_{m, \mu}\right)$ by the relation

$$
\pi^{*} i^{*} \phi_{m, \mu}=\sigma\left(\Phi_{m, \mu}\right)+\sum_{Z \subset \mathcal{Z}(m, \mu)_{U}} \log \left|\sigma\left(f_{Z}\right)\right|^{2} .
$$

The resulting arithmetic divisor

$$
\left(\sigma(\mathcal{Z}(m, \mu)), \sigma\left(\Phi_{m, \mu}\right)\right) \in \widehat{\operatorname{Div}}\left(N_{Y} U\right)
$$

does not depend on the choice of $f_{Z}$ 's used in its construction. Using étale descent these arithmetic divisors glue together to form the desired arithmetic divisor (5.5).

Proposition 5.2.6. The composition

$$
\widehat{\operatorname{Pic}}(\mathcal{M}) \stackrel{i^{*}}{\rightarrow} \widehat{\operatorname{Pic}}(\mathcal{Y}) \stackrel{\pi^{*}}{\rightarrow} \widehat{\operatorname{Pic}}\left(N_{\mathcal{Y}} \mathcal{M}\right)
$$

sends the metrized line bundle defined by $\widehat{\mathcal{Z}}(m, \mu)$ to the metrized line bundle defined by $\sigma(\widehat{\mathcal{Z}}(m, \mu))$

Proof. Let $U \rightarrow \mathcal{M}$ be a sufficiently small étale open chart, and write

$$
\mathcal{Z}(m, \mu)_{U}=\bigsqcup Z
$$

as the union of its connected components. Each connected component determines a line bundle $\mathcal{O}(Z)=f_{Z}^{-1} \mathcal{O}_{U}$, and

$$
\mathcal{Z}(m, \mu)_{U} \stackrel{\simeq}{\rightarrow} \bigotimes \mathcal{O}(Z)
$$




\section{Height Pairings on orthogonal Shimura varieties}

as line bundles on $U$.

Let $\pi^{*} i^{*} \mathcal{O}(Z)$ be the pullback of $\mathcal{O}(Z)$ via $N_{Y} U \stackrel{\pi}{\rightarrow} Y \stackrel{i}{\rightarrow} U$. If we denote by $s_{Z}$ the constant function 1 on $U$ viewed as a section of $\mathcal{O}(Z)$, then

$$
\sigma\left(s_{Z}\right)=\sigma\left(f_{Z}\right) \pi^{*} i^{*}\left(f_{Z}^{-1} s_{Z}\right)
$$

is a nonzero section of $\pi^{*} i^{*} \mathcal{O}(Z)$, and does not depend on the choice of $f_{Z}$. The tensor product $\sigma(s)=\otimes \sigma\left(s_{Z}\right)$ over all $Z$ is a global section of the metrized line bundle $\pi^{*} i^{*} \widehat{\mathcal{Z}}(m, \mu)_{U}$, and these section glue together over an étale cover of $\mathcal{M}$ to define a global section $\sigma(s)$ of $\pi^{*} i^{*} \widehat{\mathcal{Z}}(m, \mu)$.

Tracing through the definitions shows that $\widehat{\operatorname{div}}(\sigma(s))=\sigma(\widehat{\mathcal{Z}}(m, \mu))$, as arithmetic divisors, and so

$$
\pi^{*} i^{*} \widehat{\mathcal{Z}}(m, \mu) \stackrel{\simeq}{\rightarrow} \sigma(\widehat{\mathcal{Z}}(m, \mu))
$$

as metrized line bundles.

Remark 5.2.7. The construction (5.5) and Proposition 5.2.6 will be our main tools for computing improper intersections. The technique is based on the thesis of $\mathrm{J}$. $\mathrm{Hu}$ [Hu99], which reconstructs the arithmetic intersection theory of Gillet-Soulé [GS90] using Fulton's method of deformation to the normal cone.

Letting $C_{\mathcal{Y}} \mathcal{M}$ denote the normal cone of $\mathcal{Y}$ in $\mathcal{M}, \mathrm{Hu}^{4}$ constructs a specialization to the normal cone map

$$
\sigma: \widehat{\operatorname{Div}}(\mathcal{M}) \rightarrow \widehat{\operatorname{Div}}\left(C_{\mathcal{Y}} \mathcal{M}\right)
$$

and shows that

$$
[\widehat{\mathcal{Z}}: \mathcal{Y}]=[\sigma(\widehat{\mathcal{Z}}): \mathcal{Y}]
$$

for any arithmetic divisor $\widehat{\mathcal{Z}}$. Here the intersection pairing on the right is defined using the canonical closed immersion $\mathcal{Y} \rightarrow C_{\mathcal{Y}} \mathcal{M}$.

The normal bundle $N_{\mathcal{Y}} \mathcal{M}$ is essentially a first order approximation to $C_{\mathcal{Y}} \mathcal{M}$, and our construction (5.5) and Proposition 5.2.6 amount to truncating Hu's theory to first order. In order to use this to compute arithmetic intersections, it is essential to know that our divisors satisfy Lemma 5.2.5, which says, loosely speaking, that the functions $f_{Z}$ vanish along $Y$ to at most order 1. This guarantees that the specialization (5.5) to the normal bundle does not lose too much information about the divisor $\mathcal{Z}(m, \mu)$.

To be clear: we are exploiting here a special property of the divisors $\mathcal{Z}(m, \mu)$ and their relative positions with respect to $\mathcal{Y}$. It would not be very useful to define the specialization (5.5) for an arbitrary divisor on $\mathcal{M}$, as truncating Hu's specialization map to first order would lose essential higher order information.

\subsection{The cotautological bundle}

Recall from (2.10) the vector bundle $\boldsymbol{V}_{\mathrm{dR}}$ on $\mathcal{M}$ with its isotropic line $\boldsymbol{V}_{\mathrm{dR}}^{1}$, and from (4.3) the line bundle $\boldsymbol{V}_{0, \mathrm{dR}}$ on $\mathcal{Y}$. Proposition 4.6.1 implies that $\boldsymbol{V}_{0, \mathrm{dR}} \subset i^{*} \boldsymbol{V}_{\mathrm{dR}}$ in such a way that $\boldsymbol{V}_{0, \mathrm{dR}}^{1}=i^{*} \boldsymbol{V}_{\mathrm{dR}}^{1}$.

Denote by $\boldsymbol{T}=\left(\boldsymbol{V}_{\mathrm{dR}}^{1}\right)^{\vee}$ and $\boldsymbol{T}_{0}=\left(\boldsymbol{V}_{0, \mathrm{dR}}^{1}\right)^{\vee}$ the cotautological bundles on $\mathcal{M}$ and $\mathcal{Y}$, respectively, so that $\boldsymbol{T}_{0}=i^{*} \boldsymbol{T}$. Under the complex uniformization $\Gamma_{g} \backslash \mathcal{D} \rightarrow \mathcal{M}(\mathbb{C})$ of (2.3) the fiber of

${ }^{4} \mathrm{Hu}$ works in greater generality, and allows for the cycles $\mathcal{Z}$ and $\mathcal{Y}$ to have arbitrary codimension. 
$\boldsymbol{V}_{\mathrm{dR}}^{1}$ at $z \in \mathcal{D}$ is identified with the tautological line $\mathbb{C} z \subset V_{\mathbb{C}}$, which we endow with the metric

$$
\|z\|^{2}=\frac{-1}{4 \pi e^{\gamma}}[z, \bar{z}]
$$

Here, as before, $\gamma=-\Gamma^{\prime}(1)$ is the Euler-Mascheroni constant. Dualizing, we obtain a metric on the cotautological bundle, and the resulting metrized line bundle is denoted

$$
\widehat{\boldsymbol{T}} \in \widehat{\operatorname{Pic}}(\mathcal{M}) .
$$

We endow $\boldsymbol{T}_{0}$ with the unique metric for which

$$
\widehat{\boldsymbol{T}}_{0} \stackrel{\simeq}{\rightarrow} i^{*} \widehat{\boldsymbol{T}} .
$$

Proposition 5.3.1. The metrized cotautological bundle satisfies

$$
\frac{w_{\boldsymbol{k}}}{h_{\boldsymbol{k}}} \cdot[\widehat{\boldsymbol{T}}: \mathcal{Y}]=2 \frac{L^{\prime}\left(\chi_{\boldsymbol{k}}, 0\right)}{L\left(\chi_{\boldsymbol{k}}, 0\right)}+\log \left|\frac{d_{\boldsymbol{k}}}{4 \pi}\right|-\gamma
$$

Proof. This is virtually identical to the proof of [BHY15, Theorem 6.4], and so we only give an outline. Let $\mathcal{E} \rightarrow \mathcal{Y}$ be the universal $\mathcal{O}_{\boldsymbol{k}}$-elliptic curve. If we endow the $\mathcal{O}_{\mathcal{Y}}$-module $\operatorname{Lie}(\mathcal{E})$ with the Faltings metric, the Chowla-Selberg formula implies

$$
\frac{w_{\boldsymbol{k}}}{h_{\boldsymbol{k}}} \cdot \widehat{\operatorname{deg}} \widehat{\operatorname{Lie}}(\mathcal{E})=\frac{L^{\prime}\left(\chi_{\boldsymbol{k}}, 0\right)}{L\left(\chi_{\boldsymbol{k}}, 0\right)}+\log (2 \pi)+\frac{1}{2} \log \left|d_{\boldsymbol{k}}\right| .
$$

Now view $L_{0}$ as an $\mathcal{O}_{\boldsymbol{k}}$-module through left multiplication, and endow the corresponding line bundle $\boldsymbol{L}_{0}$ on $\operatorname{Spec}\left(\mathcal{O}_{\boldsymbol{k}}\right)$ with the metric

$$
\|x\|^{2}=-16 \pi^{3} e^{\gamma} \cdot Q(x) .
$$

Pulling back $\boldsymbol{L}_{0}$ to $\mathcal{Y}$ via the structure morphism yields a metrized line bundle $\widehat{\boldsymbol{L}}_{0}$ on $\mathcal{Y}$ satisfying

$$
\frac{w_{\boldsymbol{k}}}{h_{\boldsymbol{k}}} \cdot \widehat{\operatorname{deg}} \widehat{\boldsymbol{L}}_{0}=-\log \left(16 \pi^{3} e^{\gamma}\right) .
$$

By keeping track of the metrics, the isomorphism (4.4) defines an isomorphism of metrized line bundles

$$
\widehat{\boldsymbol{T}}_{0} \stackrel{\simeq}{\mathrm{Lie}}(\mathcal{E})^{\otimes 2} \otimes \widehat{\boldsymbol{L}}_{0}
$$

on $\mathcal{Y}$, and the claim follows.

Comparing with Proposition 3.3.1 proves:

Corollary 5.3.2. The coefficient $a_{L_{0}}^{+}(0) \in \mathfrak{S}_{L_{0}}^{\vee}$ satisfies

$$
a_{L_{0}}^{+}(0, \mu)=-\frac{w_{\boldsymbol{k}}}{h_{\boldsymbol{k}}} \cdot \begin{cases}{[\widehat{\boldsymbol{T}}: \mathcal{Y}]} & \text { if } \mu=0 \\ 0 & \text { otherwise }\end{cases}
$$

for every $\mu \in L_{0}^{\vee} / L_{0}$.

\subsection{The normal bundle at CM points}

Consider the orthogonal complement $\left(\boldsymbol{V}_{0, \mathrm{dR}}^{1}\right)^{\perp} \subset i^{*} \boldsymbol{V}_{\mathrm{dR}}$. Since $\mathcal{Y}$ is regular of dimension 1, this complement is torsion-free and is therefore a vector sub-bundle of $i^{*} \boldsymbol{V}_{\mathrm{dR}}$ of co-rank 1. (Note that the corresponding assertion over $\mathcal{M}$ may not be true; the orthogonal complement of $\boldsymbol{V}_{\mathrm{dR}}^{1}$ in $\boldsymbol{V}_{\mathrm{dR}}$ will in general not be a vector bundle unless $L$ is unimodular.)

Set $\mathrm{Fil}^{0} i^{*} \boldsymbol{V}_{\mathrm{dR}}=\left(\boldsymbol{V}_{0, \mathrm{dR}}^{1}\right)^{\perp}$ and $\mathrm{gr}_{\mathrm{Fil}}^{0} i^{*} \boldsymbol{V}_{\mathrm{dR}}=\mathrm{Fil}^{0} i^{*} \boldsymbol{V}_{\mathrm{dR}} / \boldsymbol{V}_{0, \mathrm{dR}}^{1}$. 


\section{Height PAIRINGs ON ORTHOGONAL ShIMURA VARIETIES}

Proposition 5.4.1. There is a canonical isomorphism of $\mathcal{O}_{\mathcal{Y}}$-modules

$$
N_{\mathcal{Y}} \mathcal{M} \stackrel{\simeq}{\longrightarrow} \boldsymbol{T}_{0} \otimes \mathrm{gr}_{\mathrm{Fil}}^{0} i^{*} \boldsymbol{V}_{\mathrm{dR}} .
$$

In particular, the normal bundle is locally free of rank $n$, and is therefore relatively representable over $\mathcal{Y}$.

Proof. Fix a sufficiently small étale open chart $U \rightarrow \mathcal{M}$, and let $Y \subset \mathcal{Y}_{U}$ be a connected component. Let $Y[\varepsilon]=Y \times_{\mathbb{Z}} \operatorname{Spec}(\mathbb{Z}[\varepsilon])$ be the scheme of dual numbers relative to $Y$, and let $\operatorname{Def}_{i}$ be the Zariski sheaf of infinitesimal deformations of $i: Y \rightarrow U$. By this we mean the sheaf associating to an open subscheme $S \subset Y$ the set $\operatorname{Def}_{i}(S)$ of morphisms $j_{S}: S[\varepsilon] \rightarrow U$ such that $\left.j_{S}\right|_{S}=i_{S}$. Here we are writing $i_{S}$ for the inclusion $S \hookrightarrow Y \hookrightarrow U$.

LEMma 5.4.2. There is a canonical isomorphism of functors

$$
\operatorname{Def}_{i} \stackrel{\simeq}{\rightarrow} N_{Y} U \text {. }
$$

Proof. The morphism (5.9) is defined in the usual way: every $j_{S} \in \operatorname{Def}_{i}(S)$ defines a map $\mathcal{O}_{U} / I^{2} \rightarrow \mathcal{O}_{S}[\varepsilon]$, whose restriction

$$
I / I^{2} \rightarrow \mathcal{O}_{S} \cdot \varepsilon \stackrel{\simeq}{\rightarrow} \mathcal{O}_{S}
$$

defines an element of $\left(N_{Y} U\right)(S)$.

To construct an inverse to (5.9), first recall that $\mathcal{Y}$ is formally étale over $\mathcal{O}_{\boldsymbol{k}}$. This implies that the surjection $\mathcal{O}_{U} / I^{2} \rightarrow \mathcal{O}_{Y}$ admits a canonical section, and so

$$
\mathcal{O}_{U} / I^{2} \stackrel{\simeq}{\rightarrow} \mathcal{O}_{Y} \oplus I / I^{2}
$$

as sheaves of rings on $\mathcal{O}_{Y}$. Any $S$-point $s \in\left(N_{Y} U\right)(S)$ therefore defines a homomorphism

$$
\mathcal{O}_{U} / I^{2} \stackrel{\simeq}{\rightarrow} \mathcal{O}_{Y} \oplus I / I^{2} \stackrel{1 \oplus s \cdot \epsilon}{\longrightarrow} \mathcal{O}_{S}[\epsilon]
$$

which in turn determines a deformation $j_{S}: S[\epsilon] \rightarrow U$ of $i_{S}$.

Lemma 5.4.3. There is a canonical isomorphism

$$
\operatorname{Def}_{i} \stackrel{\simeq}{\rightarrow}\left(\boldsymbol{V}_{0, \mathrm{dR}}^{1}\right)^{\vee} \otimes_{\mathcal{O}_{Y}} \operatorname{gr}_{\mathrm{Fil}}^{0}\left(i^{*} \boldsymbol{V}_{\mathrm{dR}}\right)
$$

of sheaves of sets on $Y$.

Proof. Let $S \subset Y$ be an open subscheme, and let $j_{S} \in \operatorname{Def}_{i}(S)$. The pull-back $j_{S}^{*} \boldsymbol{V}_{\mathrm{dR}}$ is a locally free sheaf of $\mathcal{O}_{S[\varepsilon]}$-modules of rank $n+2$, endowed with an $\mathcal{O}_{S[\varepsilon]}$-submodule $j_{S}^{*} \boldsymbol{V}_{\mathrm{dR}}^{1}$ of rank one, locally a direct summand. As $\boldsymbol{V}_{\mathrm{dR}}$ is a vector bundle with integrable connection, the retraction $S[\varepsilon] \rightarrow S$ induces a canonical isomorphism

$$
j_{S}^{*} \boldsymbol{V}_{\mathrm{dR}} \stackrel{\simeq}{\rightarrow} i_{S}^{*} \boldsymbol{V}_{\mathrm{dR}} \otimes_{\mathcal{O}_{S}} \mathcal{O}_{S[\varepsilon]}
$$

We claim that, under this isomorphism, $j_{S}^{*} \boldsymbol{V}_{\mathrm{dR}}^{1}$ maps into $\mathrm{Fil}^{0} i_{S}^{*} \boldsymbol{V}_{\mathrm{dR}} \otimes \mathcal{O}_{S} \mathcal{O}_{S[\varepsilon]}$; in other words, the image of $j_{S}^{*} \boldsymbol{V}_{\mathrm{dR}}^{1}$ is orthogonal to $i_{S}^{*} \boldsymbol{V}_{\mathrm{dR}}^{1} \otimes_{\mathcal{O}_{S}} \mathcal{O}_{S[\varepsilon]}$. This is easily deduced from the fact that both this image and $i_{S}^{*} \boldsymbol{V}_{\mathrm{dR}}^{1} \otimes_{\mathcal{O}_{S}} \mathcal{O}_{S[\varepsilon]}$ are isotropic lines lifting $i_{S}^{*} \boldsymbol{V}_{\mathrm{dR}}^{1}$.

Consider the composition

$$
j_{S}^{*} \boldsymbol{V}_{\mathrm{dR}}^{1} \hookrightarrow \mathrm{Fil}^{0} i_{S}^{*} \boldsymbol{V}_{\mathrm{dR}} \otimes_{\mathcal{O}_{S}} \mathcal{O}_{S[\varepsilon]} \rightarrow \operatorname{gr}_{\mathrm{Fil}}^{0}\left(i_{S}^{*} \boldsymbol{V}_{\mathrm{dR}}\right) \otimes_{\mathcal{O}_{S}} \mathcal{O}_{S[\varepsilon]} .
$$

The reduction of this map modulo $(\varepsilon)$ is 0 , and therefore it must factor through a map

$$
i_{S}^{*} \boldsymbol{V}_{\mathrm{dR}}^{1} \rightarrow \varepsilon \cdot\left(\operatorname{gr}_{\mathrm{Fil}}^{0}\left(i_{S}^{*} \boldsymbol{V}_{\mathrm{dR}}\right) \otimes_{\mathcal{O}_{S}} \mathcal{O}_{S[\varepsilon]}\right) \stackrel{\simeq}{\rightarrow} \operatorname{gr}_{\mathrm{Fil}}^{0}\left(i_{S}^{*} \boldsymbol{V}_{\mathrm{dR}}\right)
$$




\section{F. Andreatta, E. Z. Goren, B. Howard, K. Madapusi Pera}

Thus, we have produced a canonical morphism

$$
\operatorname{Def}_{i} \rightarrow\left(\boldsymbol{V}_{0, \mathrm{dR}}^{1}\right)^{\vee} \otimes_{\mathcal{O}_{Y}} \operatorname{gr}_{\mathrm{Fil}}^{0}\left(i^{*} \boldsymbol{V}_{\mathrm{dR}}\right)
$$

To show that this is an isomorphism, we can, by (2.4.3) and the above discussion of local models, assume that the immersion $Y \subset U$ is isomorphic to an étale neighborhood of the closed immersion $\mathrm{M}^{\mathrm{loc}}\left(L_{0}\right) \hookrightarrow \mathrm{M}^{\mathrm{loc}}(L)$. Moreover, we can choose this isomorphism in such a way that it identifies $\boldsymbol{V}_{0, \mathrm{dR}}$ and $\boldsymbol{V}_{\mathrm{dR}}$ with the trivial vector bundles $\mathbf{1} \otimes L_{0}$ and $\mathbf{1} \otimes L$, respectively, and such that $\boldsymbol{V}_{0, \mathrm{dR}}^{1}$ and $\boldsymbol{V}_{\mathrm{dR}}^{1}$ get identified with the tautological isotropic lines in $\mathbf{1} \otimes L_{0}$ and $\mathbf{1} \otimes L$, respectively.

So, if $\boldsymbol{L}_{0}^{1} \subset \mathbf{1} \otimes L_{0}$ is the tautological isotropic line over $\mathrm{M}^{\mathrm{loc}}\left(L_{0}\right)$, and $\boldsymbol{L}^{0} \subset \mathbf{1} \otimes L$ is its orthogonal complement, we are reduced to showing the elementary fact that there is a canonical isomorphism

$$
N_{\mathrm{M}^{\mathrm{loc}}\left(L_{0}\right)}\left(\mathrm{M}^{\mathrm{loc}}(L)\right) \stackrel{\simeq}{\rightarrow} \underline{\operatorname{Hom}}\left(\boldsymbol{L}_{0}^{1}, \boldsymbol{L}^{0} / \boldsymbol{L}_{0}^{1}\right)
$$

of Zariski sheaves over $\mathrm{M}^{\mathrm{loc}}\left(L_{0}\right)$. We leave this as an exercise to the reader.

The proof of Proposition 5.4.1 now follows by combining Lemmas (5.4.2) and (5.4.3) and glueing over an étale cover of $\mathcal{M}$.

We will have use for the following composition:

$$
N_{\mathcal{Y}} \mathcal{M} \underset{(5.4 .1)}{\stackrel{\simeq}{\longrightarrow}} \boldsymbol{T}_{0} \otimes \operatorname{gr}_{\mathrm{Fil}}^{0} i^{*} \boldsymbol{V}_{\mathrm{dR}} \rightarrow \boldsymbol{T}_{0} \otimes\left(i^{*} \boldsymbol{V}_{\mathrm{dR}} / \boldsymbol{V}_{0, \mathrm{dR}}\right)
$$

Here, the map on the right is induced by the natural map

$$
\operatorname{gr}_{\mathrm{Fil}}^{0} i^{*} \boldsymbol{V}_{\mathrm{dR}} \rightarrow i^{*} \boldsymbol{V}_{\mathrm{dR}} / \boldsymbol{V}_{\mathrm{dR}}^{0}
$$

Note that this last map is an isomorphism over the generic fiber of $\mathcal{Y}$. Indeed, it amounts to knowing that the inclusion $\boldsymbol{V}_{0, \mathrm{dR}}+\mathrm{Fil}^{0} i^{*} \boldsymbol{V}_{\mathrm{dR}} \subset i^{*} \boldsymbol{V}_{\mathrm{dR}}$ is an isomorphism over the generic fiber. This follows from the self-duality of the quadratic form on $\boldsymbol{V}_{\mathrm{dR}, M}$.

On the other hand, over the generic fiber, the map $\boldsymbol{\Lambda}_{\mathrm{dR}} \rightarrow i^{*} \boldsymbol{V}_{\mathrm{dR}} / \boldsymbol{V}_{\mathrm{dR}}^{0}$ is also an isomorphism. Therefore, for any point $y \in \mathcal{Y}(\mathbb{C}),(5.10)$ induces a canonical isomorphism

$$
\left(N_{\mathcal{Y}} \mathcal{M}\right)_{y} \stackrel{\simeq}{\rightarrow} \boldsymbol{T}_{0, y} \otimes \Lambda_{\mathbb{C}} .
$$

We will now give an explicit description of this isomorphism. To begin, $y$ determines an isotropic line $\mathbb{C} y \subset L_{\mathbb{C}}$, whose dual can be naturally identified with the fiber $\boldsymbol{T}_{0, y}$ of $\boldsymbol{T}_{0}$ at $y$. The construction of the isomorphism (5.11) proceeded by choosing an appropriate local trivialization of $\boldsymbol{V}_{\mathrm{dR}}$. In an analytic neighborhood of $y$, this simply means that we choose a local section $U \rightarrow \mathcal{D}$ of the complex analytic uniformization carrying $y$ to $\mathbb{C} y$.

Note that $\mathcal{D}$ is contained in the complex quadric $\mathrm{M}^{\mathrm{loc}}\left(L_{\mathbb{C}}\right)$. We therefore see that it is enough to describe the induced isomorphism:

$$
T_{\mathbb{C} y} \mathcal{D}=T_{\mathbb{C} y} \mathrm{M}^{\mathrm{loc}}\left(L_{\mathbb{C}}\right) \stackrel{\simeq}{\rightarrow}(\mathbb{C} y)^{\vee} \otimes \Lambda_{\mathbb{C}}
$$

Here, $T_{\mathbb{C} y} \mathcal{D}$ is the tangent space of $\mathcal{D}$ at $\mathbb{C} y$.

We consider the local immersion

$$
\operatorname{Hom}\left(\mathbb{C} y, \Lambda_{\mathbb{C}}\right)=(\mathbb{C} y)^{\vee} \otimes \Lambda_{\mathbb{C}} \rightarrow \mathrm{M}^{\mathrm{loc}}\left(L_{\mathbb{C}}\right),
$$




\section{Height PAIRINGS ON ORTHOGONAL SHIMURA VARIETIES}

which carries a section $\xi$ to the isotropic line spanned by

$$
y+\xi(y)-\frac{Q(\xi(y))}{[y, \bar{y}]} \bar{y} .
$$

This carries 0 to $\mathbb{C} y$, and the derivative at 0 is exactly the inverse to (5.12).

\subsection{Specialization of the Green function}

Suppose we have a complex point $y \in \mathcal{Y}(\mathbb{C})$. Under the uniformization

$$
\mathcal{Y}(\mathbb{C}) \stackrel{\simeq}{\rightarrow} T(\mathbb{Q}) \backslash\left\{\boldsymbol{h}_{0}\right\} \times T\left(\mathbb{A}_{f}\right) / \widehat{\mathcal{O}}_{\boldsymbol{k}}^{\times}
$$

of $\S 4.2$, the point $y$ is represented by a pair $\left(\boldsymbol{h}_{0}, g\right)$ with

$$
g \in T\left(\mathbb{A}_{f}\right) \subset G\left(\mathbb{A}_{f}\right),
$$

and so its image in $\mathcal{M}(\mathbb{C})$ lies on the component $\Gamma_{g} \backslash \mathcal{D}$ of $(2.3)$. Here $G=\operatorname{GSpin}(L)$. By mild abuse of notation we also let $\mathbb{C} y \in \mathcal{D}$ denote the isotropic line corresponding to the oriented negative plane $\boldsymbol{h}_{0}=L_{0 \mathbb{R}}$, so that $\mathbb{C} y$ is a lift of $y$ under $\mathcal{D} \rightarrow \mathcal{M}(\mathbb{C})$, and $L_{\mathbb{C}}=\mathbb{C} y \oplus \mathbb{C} \bar{y} \oplus \Lambda_{\mathbb{C}}$.

From (2.4), we find that a neighborhood of $y$ admits a complex analytic uniformization

$$
\bigsqcup_{\substack{x \in \mu_{g}+L_{g} \\ Q(x)=m}} \mathcal{D}(x) \rightarrow \mathcal{Z}(m, \mu)(\mathbb{C}) .
$$

The components $\mathcal{D}(x)$ passing through $\mathbb{C} y$ are precisely those for which $x$ is orthogonal to $y$. But, since $x$ is rational, this is equivalent to requiring that $x$ be orthogonal to both $y$ and $\bar{y}$; or, in other words, that $x$ lies in $\Lambda^{\vee}$.

As the orthogonal transformation $g \in \mathrm{SO}\left(L \otimes \mathbb{A}_{f}\right)$ acts trivially on the direct summand $\Lambda \otimes \mathbb{A}_{f}$, we see that such $x$ in fact lie in $\mu+L$. This shows that in a small enough analytic neighborhood $U$ of $\mathbb{C} y$ there is an isomorphism of divisors

$$
U \cap \sum_{\lambda \in \Lambda_{m, \mu}} \mathcal{D}(\lambda) \stackrel{\simeq}{\rightarrow} U \cap \mathcal{Z}(m, \mu)(\mathbb{C}),
$$

where

$$
\Lambda_{m, \mu}=\left\{\lambda \in \Lambda^{\vee}: \begin{array}{l}
Q(\lambda)=m \\
\lambda \in \mu+L
\end{array}\right\} .
$$

Recall that each isotropic line $\mathbb{C} z \in \mathcal{D}$ corresponds to a negative plane $\boldsymbol{h}_{z} \subset V_{\mathbb{R}}$. For each $\lambda \in \Lambda_{m, \mu}$ let $\lambda_{z} \in V_{\mathbb{R}}$ be the orthogonal projection of $\lambda$ to $\boldsymbol{h}_{z}$. Simple linear algebra gives

$$
Q\left(\lambda_{z}\right)=\frac{[\lambda, \bar{z}] \cdot[\lambda, z]}{[z, \bar{z}]}
$$

Define a function on $U \backslash \mathcal{Z}(m, \mu)(\mathbb{C})$ by

$$
\Phi_{m, \mu}^{\mathrm{reg}}=\Phi_{m, \mu}+\sum_{\lambda \in \Lambda_{m, \mu}} \log \left|4 \pi e^{\gamma} \cdot Q\left(\lambda_{z}\right)\right| .
$$

Recall that the function $\Phi_{m, \mu}(z)$ is defined, but discontinuous, at $z=y$. The following proposition gives us a method of computing the value of this function at the discontinuity $z=y$.

Proposition 5.5.1. The function $\Phi_{m, \mu}^{\mathrm{reg}}$ extends smoothly to all of $U$, and this extension satisfies

$$
\Phi_{m, \mu}^{\mathrm{reg}}(y)=\Phi_{m, \mu}(y)
$$




\section{F. Andreatta, E. Z. Goren, B. Howard, K. Madapusi Pera}

Proof. Let $\tau=u+i v \in \mathcal{H}$ be the variable on the upper half-plane. The function $\Phi_{m, \mu}(z)$ is defined, as in [BY09, (4.8)], as the constant term of the Laurent expansion at $s=0$ of

$$
\lim _{T \rightarrow \infty} \int_{\mathcal{F}_{T}}\left\{F_{m, \mu}(\tau), \theta_{L}(\tau, z)\right\} \frac{d u d v}{v^{s+2}},
$$

where

$$
\mathcal{F}_{T}=\{\tau=u+i v \in \mathcal{H}:|u| \leqslant 1 / 2,|\tau| \geqslant 1, v \leqslant T\} .
$$

Now substitute the definition [BY09, $(2.4)$ ] of $\theta_{L}(\tau, z)$ and the Fourier expansion of $F_{m, \mu}$ into (5.14), and argue as in [Bor98, Theorem 6.2]. Using the decomposition $F_{m, \mu}=F_{m, \mu}^{+}+F_{m, \mu}^{-}$of [BY09, (3.4a) and (3.4b)] and the rapid decay as $v \rightarrow \infty$ of the Fourier coefficients of $F_{m, \mu}^{-}$, we find

$$
\Phi_{m, \mu}(z)=\varphi_{m, \mu}(z)+\sum_{\lambda \in \Lambda_{m, \mu}} \mathrm{CT}_{s=0}\left[\int_{1}^{\infty} e^{4 \pi v Q\left(\lambda_{z}\right)} \frac{d v}{v^{s+1}}\right]
$$

for some smooth function $\varphi_{m, \mu}$ on $U$, where $\mathrm{CT}_{s=0}$ means take the constant term at $s=0$.

Each $\lambda \in \Lambda_{m, \mu}$ is orthogonal to $y$, and so $Q\left(\lambda_{y}\right)=0$. Thus setting $z=y$ in (5.15) and computing the constant term at $s=0$ shows that

$$
\Phi_{m, \mu}(y)=\varphi_{m, \mu}(y) .
$$

On the other hand, for any $z \in U \backslash \mathcal{Z}(m, \mu)(\mathbb{C})$ we have $Q\left(\lambda_{z}\right)<0$ for every $\lambda \in \Lambda_{m, \mu}$, and so

$$
\begin{aligned}
\sum_{\lambda \in \Lambda_{m}, \mu} \mathrm{CT}_{s=0}\left[\int_{1}^{\infty} e^{4 \pi v Q\left(\lambda_{z}\right)} \frac{d v}{v^{s+1}}\right] & =\sum_{\lambda \in \Lambda_{m, \mu}} \int_{1}^{\infty} e^{4 \pi v Q\left(\lambda_{z}\right)} \frac{d v}{v} \\
& =\sum_{\lambda \in \Lambda_{m, \mu}} \Gamma\left(0,-4 \pi Q\left(\lambda_{z}\right)\right)
\end{aligned}
$$

where

$$
\Gamma(0, x)=\int_{x}^{\infty} e^{-t} \frac{d t}{t}=-\gamma-\log (x)-\sum_{k=1}^{\infty} \frac{(-x)^{k}}{k \cdot k !} .
$$

Comparing (5.16) with (5.17) shows that

$$
\sum_{\lambda \in \Lambda_{m, \mu}} \log \left|4 \pi e^{\gamma} \cdot Q\left(\lambda_{z}\right)\right|=-\sum_{\lambda \in \Lambda_{m, \mu}} \mathrm{CT}_{s=0}\left[\int_{1}^{\infty} e^{4 \pi v Q\left(\lambda_{z}\right)} \frac{d v}{v^{s+1}}\right]
$$

up to a smooth function on $U$, vanishing at $z=y$. Adding this equality to (5.15) proves that

$$
\Phi_{m, \mu}^{\mathrm{reg}}(z)=\varphi_{m, \mu}(z)
$$

up to a smooth function vanishing at $z=y$, and completes the proof.

For any $y \in \mathcal{Y}(\mathbb{C})$, we have a canonical isomorphism (see 5.11)

$$
\left(N_{\mathcal{Y}} \mathcal{M}\right)_{y} \stackrel{\simeq}{\rightarrow}(\mathbb{C} y)^{\vee} \otimes_{\mathbb{C}} \Lambda_{\mathbb{C}}=\operatorname{Hom}\left(\mathbb{C} y, \Lambda_{\mathbb{C}}\right) .
$$

For each $\lambda \in \Lambda_{m, \mu}$, define a function $\Phi_{\lambda}$ on $\operatorname{Hom}\left(\mathbb{C} y, \Lambda_{\mathbb{C}}\right)$ by

$$
\Phi_{\lambda}(\xi)=-\log \left|\frac{4 \pi e^{\gamma}[\lambda, \xi(y)]^{2}}{[y, \bar{y}]}\right| .
$$




\section{Height Pairings on orthogonal Shimura varieties}

This function has a logarithmic singularity along the hyperplane $(\mathbb{C} y)^{\vee} \otimes \lambda^{\perp}$. Letting $y$ vary over $\mathcal{Y}(\mathbb{C})$ yields a function $\Phi_{\lambda}$ on the vector bundle $\left(N_{\mathcal{Y}} \mathcal{M}\right)(\mathbb{C})$ having a logarithmic singularity along a sub-bundle of hyperplanes.

Proposition 5.5.2. The Green function $\sigma\left(\Phi_{m, \mu}\right)$ defined by (5.7) satisfies

$$
\sigma\left(\Phi_{m, \mu}\right)=\pi^{*} i^{*} \Phi_{m, \mu}+\sum_{\lambda \in \Lambda_{m, \mu}} \Phi_{\lambda}
$$

where $\pi^{*} i^{*} \Phi_{m, \mu}$ denotes the pullback of $\Phi_{m, \mu}$ via

$$
\left(N_{\mathcal{Y}} \mathcal{M}\right)(\mathbb{C}) \stackrel{\pi}{\rightarrow} \mathcal{Y}(\mathbb{C}) \stackrel{i}{\rightarrow} \mathcal{M}(\mathbb{C}) .
$$

Proof. As above, fix a point $y \in \mathcal{Y}(\mathbb{C})$ and let $U \subset \mathcal{D}$ be a neighborhood of the isotropic line $\mathbb{C} y \subset L_{\mathbb{C}}$. As in (5.4), we can define a holomorphic local immersion

$$
\operatorname{Hom}\left(\mathbb{C} y, \Lambda_{\mathbb{C}}\right)=(\mathbb{C} y)^{\vee} \otimes \Lambda_{\mathbb{C}} \rightarrow \mathrm{M}^{\mathrm{loc}}\left(L_{\mathbb{C}}\right)
$$

by sending $\xi$ to the $\mathbb{C}$-span of

$$
y+\xi(y)-\frac{Q(\xi(y)) \bar{y}}{[y, \bar{y}]} .
$$

As we saw at the end of (5.4), the induced isomorphism on tangent spaces

$$
(\mathbb{C} y)^{\vee} \otimes \Lambda_{\mathbb{C}} \rightarrow T_{y} \mathcal{D} \stackrel{\simeq}{\rightarrow}\left(N_{\mathcal{Y}} \mathcal{M}\right)_{y}
$$

agrees with the isomorphism of (5.18).

From now on we use the above map to identify $U$ with an open neighborhood of the origin of $(\mathbb{C} y)^{\vee} \otimes \Lambda_{\mathbb{C}}$. Each divisor $\mathcal{D}(\lambda) \cap U$ appearing in (5.13) is identified with the zero locus of

$$
f_{\lambda}(\xi)=[\lambda, \xi(y)] \cdot\left|\frac{4 \pi e^{\gamma}}{[y, \bar{y}]}\right|^{1 / 2} .
$$

This function is already linear, and so when we apply the construction $f_{\lambda} \mapsto \sigma\left(f_{\lambda}\right)$ of the discussion preceding (5.7) and identify $(\mathbb{C} y)^{\vee} \otimes \Lambda_{\mathbb{C}}$ with its own tangent space at the origin, we obtain

Thus $-\log \left|\sigma\left(f_{\lambda}\right)\right|^{2}=\Phi_{\lambda}$.

$$
\sigma\left(f_{\lambda}\right)(\xi)=[\lambda, \xi(\boldsymbol{v})] \cdot\left|\frac{4 \pi e^{\gamma}}{[\boldsymbol{v}, \overline{\boldsymbol{v}}]}\right|^{1 / 2}
$$

If we define a smooth function on $U$ by

$$
\phi_{m, \mu}=\Phi_{m, \mu}+\sum_{\lambda \in \Lambda_{m, \mu}} \log \left|f_{\lambda}\right|^{2},
$$

then directly from the definition (5.7) and the paragraph above we see that

$$
\sigma\left(\Phi_{m, \mu}\right)=\pi^{*} i^{*} \phi_{m, \mu}+\sum_{\lambda \in \Lambda_{m, \mu}} \Phi_{\lambda}
$$

Now use $\phi_{m, \mu}(y)=\Phi_{m, \mu}^{\mathrm{reg}}(y)=\Phi_{m, \mu}(y)$ to complete the proof.

\subsection{The calculation of the pullback}

Recall that the Kuga-Satake abelian scheme $\mathcal{A} \rightarrow \mathcal{M}$ restricts to the abelian scheme

$$
\mathcal{A}_{\mathcal{Y}}=\mathcal{E} \otimes_{C^{+}\left(L_{0}\right)} C(L)
$$




\section{F. Andreatta, E. Z. Goren, B. Howard, K. Madapusi Pera}

over $\mathcal{Y}$, where $\mathcal{E} \rightarrow \mathcal{Y}$ is the universal elliptic curve.

By Proposition 4.6.3, every $\lambda \in \Lambda_{m, \mu}$ determines a canonical special endomorphism $\boldsymbol{\ell}_{\lambda} \in$ $V_{\mu}\left(\mathcal{A}_{\mathcal{Y}}\right)$, and the pair $\left(\mathcal{A}_{\mathcal{Y}}, \boldsymbol{\ell}_{\lambda}\right)$ is a $\mathcal{Y}$-valued point of $\mathcal{Z}(m, \mu)$. The corresponding map $\mathcal{Y} \rightarrow$ $\mathcal{Z}(m, \mu)$ is finite and unramified, and so we may define an $\mathcal{O}_{\mathcal{Y}}$-submodule

$$
\mathcal{Z}_{\lambda}=N_{\mathcal{Y}} \mathcal{Z}(m, \mu)
$$

of $N_{\mathcal{Y}} \mathcal{M}$ in exactly the same way that $N_{\mathcal{Y}} \mathcal{M}$ was defined.

Proposition 4.6.1 provides us with a canonical morphism $\Lambda \rightarrow i^{*} \boldsymbol{V}_{\mathrm{dR}}$ such that $\Lambda^{\perp}=\boldsymbol{V}_{0, \mathrm{dR}} \subset$ $i^{*} \boldsymbol{V}_{\mathrm{dR}}$. Thus, the bilinear pairing on $i^{*} \boldsymbol{V}_{\mathrm{dR}}$ gives us a map

$$
i^{*} \boldsymbol{V}_{\mathrm{dR}} / \boldsymbol{V}_{0, \mathrm{dR}} \rightarrow \mathcal{O}_{\mathcal{Y}} \otimes \Lambda^{\vee}
$$

of vector bundles over $\mathcal{Y}$. This map is injective, and using local trivializations, one sees that its image is identified precisely with the inclusion

$$
\mathcal{O}_{\mathcal{Y}} \otimes\left(L / L_{0}\right) \hookrightarrow \mathcal{O}_{\mathcal{Y}} \otimes \Lambda^{\vee}
$$

This latter inclusion is the natural one induced by the bilinear pairing on $L$.

Now, by definition, $\Lambda_{m, \mu}$ is contained in $\left(L / L_{0}\right)^{\vee} \subset L^{\vee}$. Therefore, we obtain a morphism:

of $\mathcal{O}_{\mathcal{Y}}$-modules.

$$
[\cdot, \lambda]: i^{*} \boldsymbol{V}_{\mathrm{dR}} / \boldsymbol{V}_{0, \mathrm{dR}} \rightarrow \mathcal{O}_{\mathcal{Y}} \otimes\left(L / L_{0}\right) \stackrel{1 \otimes \lambda}{\longrightarrow} \mathcal{O}_{\mathcal{Y}}
$$

Here is another way to describe this morphism. Recall from $\S 4.6$ that we have a canonical

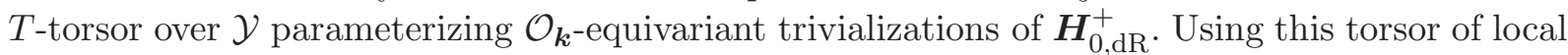
trivializations, we get a canonical functor from representations of $T$ to vector bundles over $\mathcal{Y}$ with integrable connection.

The map $[\cdot, \lambda]$ is now simply obtained by applying this functor to the $T$-invariant functional $L / L_{0} \rightarrow \mathbb{Z}$ given by pairing with $\lambda$. In particular, if $t_{\mathrm{dR}}\left(\boldsymbol{\ell}_{\lambda}\right)$ is the de Rham realization of $\boldsymbol{\ell}_{\lambda}$ (it is a section of $i_{S}^{*} \boldsymbol{V}_{\mathrm{dR}}^{\vee}$ ), we find that functionals

$$
i_{S}^{*} \boldsymbol{V}_{\mathrm{dR}} \rightarrow i_{S}^{*} \boldsymbol{V}_{\mathrm{dR}} / \boldsymbol{V}_{0, \mathrm{dR}} \stackrel{[\cdot, \lambda]}{\longrightarrow} \mathcal{O}_{S}
$$

and

$$
\left[\cdot, t_{\mathrm{dR}}\left(\boldsymbol{\ell}_{\lambda}\right)\right]: i_{S}^{*} \boldsymbol{V}_{\mathrm{dR}} \rightarrow \mathcal{O}_{S}
$$

are identical.

Proposition 5.6.1. Fix any $\lambda \in \Lambda_{m, \mu}$.

(i) The divisor $\mathcal{Z}_{\lambda}$ is canonically identified with the kernel of

$$
N_{\mathcal{Y}} \mathcal{M} \stackrel{(5.10)}{\longrightarrow} \boldsymbol{T}_{0} \otimes\left(i^{*} \boldsymbol{V}_{\mathrm{dR}} / \boldsymbol{V}_{0, \mathrm{dR}}\right) \stackrel{[\cdot, \lambda]}{\longrightarrow} \boldsymbol{T}_{0} .
$$

(ii) The metrized line bundle $\pi^{*} \widehat{\boldsymbol{T}}_{0}$ is represented by the arithmetic divisor

$$
\left(\mathcal{Z}_{\lambda}, \Phi_{\lambda}\right) \in \widehat{\operatorname{Div}}\left(N_{\mathcal{Y}} \mathcal{M}\right) \text {. }
$$

Proof. We work over a sufficiently small étale neighborhood $Y \subset U$ of $\mathcal{Y} \rightarrow \mathcal{M}$. Suppose that we are given an open subspace $S \subset Y$ and $j_{S} \in \operatorname{Def}_{i}(S)$. By the construction of the map (5.10), to prove (1), we have to show that $\boldsymbol{\ell}_{\lambda}$ lifts to an element in $V_{\mu}\left(j_{S}^{*} \mathcal{A}\right)$ if and only if the composition

$$
j_{S}^{*} \boldsymbol{V}_{\mathrm{dR}}^{1} \rightarrow i_{S}^{*} \boldsymbol{V}_{\mathrm{dR}} / \boldsymbol{V}_{0, \mathrm{dR}} \otimes_{\mathcal{O}_{S}} \mathcal{O}_{S[\varepsilon]} \stackrel{[\cdot, \lambda]}{\longrightarrow} \mathcal{O}_{S[\varepsilon]}
$$




\section{Height PAIRINGs on ORTHOgOnal Shimura VARIETIES}

is identically 0 . This follows from Lemma 2.7.5 and the identification we made above of $[\cdot, \lambda]$ with pairing against $t_{\mathrm{dR}}\left(\boldsymbol{\ell}_{\lambda}\right)$.

The second claim follows from the first, as the map $[\cdot, \lambda]: N_{\mathcal{Y}} \mathcal{M} \rightarrow \boldsymbol{T}_{0}$ defines a section $s_{\lambda}$ of $\pi^{*} \boldsymbol{T}_{0}$ with $\widehat{\operatorname{div}}\left(s_{\lambda}\right)=\left(\mathcal{Z}_{\lambda}, \Phi_{\lambda}\right)$. Indeed, using the notation of (5.18), the norm of this section at a complex point $\xi \in\left(N_{\mathcal{Y}} \mathcal{M}\right)_{y}$ in the fiber over $y \in \mathcal{Y}(\mathbb{C})$ is

$$
-\log \left\|s_{\lambda}\right\|^{2}=-\log \left|\frac{[\lambda, \xi(y)]}{\|y\|}\right|^{2}=\Phi_{\lambda}(\xi),
$$

where the second equality is by definition (5.8) of the metric on $\widehat{\boldsymbol{T}}_{0}$.

As in $[\mathrm{BHY} 15, \S 7.3]$, we define a new metrized line bundle

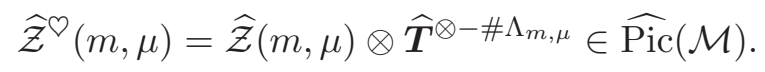

Proposition 5.6.2. The image of $\widehat{\mathcal{Z}}^{\ominus}(m, \mu)$ under the pullback

$$
\widehat{\operatorname{Pic}}(\mathcal{M}) \stackrel{i^{*}}{\rightarrow} \widehat{\operatorname{Pic}}(\mathcal{Y})
$$

is represented by the arithmetic divisor

$$
\left(\sum_{\substack{m_{1}>0 \\ m_{2} \geq 0 \\ m_{1}+m_{2}=m}} \sum_{\substack{\mu_{1} \in L_{0}^{\vee} / L_{0} \\ \mu_{2} \in \Lambda^{\vee} / \Lambda \\\left(\mu_{1}, \mu_{2}\right) \in(\mu+L) /\left(L_{0} \oplus \Lambda\right)}} R_{\Lambda}\left(m_{2}, \mu_{2}\right) \cdot \mathcal{Z}_{0}\left(m_{1}, \mu_{1}\right), i^{*} \Phi_{m, \mu}\right) \in \widehat{\operatorname{Div}}(\mathcal{Y})
$$

Proof. By Proposition 4.6.4 there is a decomposition of $\mathcal{Y}$-stacks

$$
\mathcal{Z}(m, \mu) \times \mathcal{M} \mathcal{Y}=\bigsqcup_{\substack{m_{1}, m_{2} \in \mathbb{Q} \geqslant 0 \\ m_{1}+m_{2}=m}} \bigsqcup_{\substack{\mu_{1} \in L_{0}^{\vee} / L_{0} \\ \mu_{2} \in \Lambda^{\vee} / \Lambda \\\left(\mu_{1}, \mu_{2}\right) \in(\mu+L) /\left(L_{0} \oplus \Lambda\right)}} \mathcal{Z}_{0}\left(m_{1}, \mu_{1}\right) \times \Lambda_{m_{2}, \mu_{2}} .
$$

Using Remark 4.6.5 we may therefore decompose

$$
\mathcal{Z}(m, \mu) \times_{\mathcal{M}} \mathcal{Y}=\mathcal{Z}_{0}^{\text {prop }} \sqcup\left(\mathcal{Y} \times \Lambda_{m, \mu}\right),
$$

where

$$
\mathcal{Z}_{0}^{\text {prop }}=\bigsqcup_{\substack{m_{1}>0 \\ m_{2} \geqslant 0 \\ m_{1}+m_{2}=m}} \bigsqcup_{\substack{\mu_{1} \in L_{0}^{\vee} / L_{0} \\ \mu_{2} \in \Lambda^{\vee} / \Lambda \\\left(\mu_{1}, \mu_{2}\right) \in(\mu+L) /\left(L_{0} \oplus \Lambda\right)}} \mathcal{Z}_{0}\left(m_{1}, \mu_{1}\right) \times \Lambda_{m_{2}, \mu_{2}}
$$

is a $\mathcal{Y}$-stack of dimension 0 . Moreover, the induced map

$$
\mathcal{Y} \times \Lambda_{m, \mu} \rightarrow \mathcal{Z}(m, \mu)
$$

determines, for each $\lambda \in \Lambda_{m, \mu}$, a map $\mathcal{Y} \rightarrow \mathcal{Z}(m, \mu)$, which is none other than the map used in the definition (5.19) of $\mathcal{Z}_{\lambda}$.

Over a sufficiently small étale open chart $U \rightarrow \mathcal{M}$ we may fix a connected component $Y \subset \mathcal{Y}_{U}$, and decompose

$$
\mathcal{Z}(m, \mu)_{U}=Z^{\text {prop }} \sqcup\left(Y \times \Lambda_{m, \mu}\right)
$$

where $Z^{\text {prop }}$ is the union of all connected components $Z \subset \mathcal{Z}(m, \mu)_{U}$ for which $Z \cap Y$ has dimension 0. If we apply the specialization to the normal bundle construction of (5.6) separately to $Z^{\text {prop }}$ and to each of the $\# \Lambda_{m, \mu}$ copies of $Y$, and then glue over an étale cover, we find the 
equality

$$
\sigma(\mathcal{Z}(m, \mu))=\pi^{*} \mathcal{Z}_{0}^{\text {prop }}+\sum_{\lambda \in \Lambda_{m, \mu}} \mathcal{Z}_{\lambda}
$$

of divisors on $N_{\mathcal{Y}} \mathcal{M}$.

By Propositions 5.2.6 and 5.6.1 the image of $\widehat{\mathcal{Z}}^{\Upsilon}(m, \mu)$ under

$$
\widehat{\operatorname{Pic}}(\mathcal{M}) \stackrel{i^{*}}{\rightarrow} \widehat{\operatorname{Pic}}(\mathcal{Y}) \stackrel{\pi^{*}}{\rightarrow} \widehat{\operatorname{Pic}}\left(N_{\mathcal{Y}} \mathcal{M}\right)
$$

is

$$
\pi^{*} i^{*} \widehat{\mathcal{Z}}^{\bigcirc}(m, \mu)=\sigma(\widehat{\mathcal{Z}}(m, \mu))-\sum_{\lambda \in \Lambda_{m, \mu}}\left(\mathcal{Z}_{\lambda}, \Phi_{\lambda}\right),
$$

and Proposition 5.5.2 and (5.20) allow us to rewrite this as an equality of metrized line bundles

$$
\pi^{*} i^{*} \widehat{\mathcal{Z}}^{\bigodot}(m, \mu)=\pi^{*}\left(\mathcal{Z}_{0}^{\text {prop }}, i^{*} \Phi_{m, \mu}\right)
$$

on $N_{\mathcal{Y}} \mathcal{M}$. Pulling back by the zero section $\mathcal{Y} \rightarrow N_{\mathcal{Y}} \mathcal{M}$ yields an isomorphism

$$
i^{*} \widehat{\mathcal{Z}}^{\bigcirc}(m, \mu)=\left(\mathcal{Z}_{0}^{\text {prop }}, i^{*} \Phi_{m, \mu}\right)
$$

of metrized line bundles on $\mathcal{Y}$.

\subsection{The main result}

We are now ready to prove our main result. For the reader's convenience, we review the dramatis personae.

The lattices $L_{0} \oplus \Lambda \subset L$ determine a finite and unramified morphism of stacks $\mathcal{Y} \rightarrow \mathcal{M}$ over $\mathcal{O}_{\boldsymbol{k}}$, and hence a linear functional

$$
[\cdot: \mathcal{Y}]: \widehat{\mathrm{CH}}^{1}(\mathcal{M}) \rightarrow \mathbb{R}
$$

defined in $\S 5.1$.

Fix a weak harmonic Maass form $f(\tau) \in H_{1-n / 2}\left(\omega_{L}\right)$. The holomorphic part

$$
f^{+}(\tau)=\sum_{m \gg-\infty} c_{f}^{+}(m) \cdot q^{m}
$$

is a formal $q$-expansion valued in the finite-dimensional vector space $\mathfrak{S}_{L}$ of complex functions on $L^{\vee} / L$. We assume that $f^{+}$has integral principal part, so that the constructions of $\S 3.2$ provide us with an arithmetic divisor

$$
\widehat{\mathcal{Z}}(f)=(\mathcal{Z}(f), \Phi(f)) \in \widehat{\mathrm{CH}}^{1}(\mathcal{M}) .
$$

We also have, from $§ 5.3$, the metrized cotautological bundle

$$
\widehat{\boldsymbol{T}} \in \widehat{\mathrm{CH}}^{1}(\mathcal{M}) \text {. }
$$

Recalling the Bruinier-Funke differential operator

$$
\xi: H_{1-\frac{n}{2}}\left(\omega_{L}\right) \rightarrow S_{1+\frac{n}{2}}\left(\bar{\omega}_{L}\right)
$$

from (3.1), we may form the convolution $L$-function $L\left(\xi(f), \Theta_{\Lambda}, s\right)$ of $\xi(f)$ with the theta series $\Theta_{\Lambda}(\tau) \in M_{\frac{n}{2}}\left(\omega_{\Lambda}^{\vee}\right)$ of $\S 3.3$. The convolution $L$-function vanishes at $s=0$, the center of the functional equation. 


\section{Height PAIRINGS ON ORTHOGONAL SHIMURA VARIETIES}

Recall the $\mathfrak{S}_{L_{0} \oplus \Lambda}$-valued formal $q$-expansion $\mathcal{E}_{L_{0}} \otimes \Theta_{\Lambda}$ of $\S 3.3$. Using (3.2) and (3.3), we obtain a scalar-valued $q$-expansion

$$
\left\{f^{+}, \mathcal{E}_{L_{0}} \otimes \Theta_{\Lambda}\right\}=\sum_{m_{1}, m_{2}, m_{3} \in \mathbb{Q}}\left\{c_{f}^{+}\left(m_{1}\right), a_{L_{0}}^{+}\left(m_{2}\right) \otimes R_{\Lambda}\left(m_{3}\right)\right\} \cdot q^{m_{1}+m_{2}+m_{3}}
$$

with constant term

$$
\mathrm{CT}\left\{f^{+}, \mathcal{E}_{L_{0}} \otimes \Theta_{\Lambda}\right\}=\sum_{m_{1}+m_{2}+m_{3}=0}\left\{c_{f}^{+}\left(m_{1}\right), a_{L_{0}}^{+}\left(m_{2}\right) \otimes R_{\Lambda}\left(m_{3}\right)\right\} .
$$

The following CM value formula was proved by Schofer [Sch09] for weakly holomorphic modular forms, and then generalized to harmonic weak Maass forms by Bruinier-Yang [BY09]. We repeat here the statement of [BY09, Theorem 4.7], with a sign error corrected. See also [BHY15, Theorem 5.3.6].

Theorem 5.7.1 Schofer, Bruinier-Yang. The Green function $\Phi(f)$ satisfies

$$
\frac{w_{\boldsymbol{k}}}{h_{\boldsymbol{k}}} \sum_{y \in \mathcal{Y}(\mathbb{C})} \frac{\Phi(f, y)}{\# \operatorname{Aut}(y)}=-L^{\prime}\left(\xi(f), \Theta_{\Lambda}, 0\right)+\mathrm{CT}\left\{f^{+}, \mathcal{E}_{L_{0}} \otimes \Theta_{\Lambda}\right\},
$$

where

$$
\xi: H_{1-\frac{n}{2}}\left(\omega_{L}\right) \rightarrow S_{1+\frac{n}{2}}\left(\bar{\omega}_{L}\right)
$$

is the Bruinier-Funke differential operator of (3.1).

Remark 5.7.2. Recalling Remark 3.2.1, Theorem 5.7.1 holds even when some points of $\mathcal{Y}(\mathbb{C})$ lie on $\mathcal{Z}(f)(\mathbb{C})$, the divisor along which $\Phi(f)$ has its logarithmic singularities.

Inspired by Theorem 5.7.1, the following formula was conjectured by Bruinier-Yang [BY09].

Theorem 5.7.3. Assume $d_{\boldsymbol{k}}$ is odd. Every weak harmonic Maass form $f \in H_{1-n / 2}\left(\omega_{L}\right)$ with integral principal part satisfies

$$
[\widehat{\mathcal{Z}}(f): \mathcal{Y}]+c_{f}^{+}(0,0) \cdot[\widehat{\boldsymbol{T}}: \mathcal{Y}]=-\frac{h_{\boldsymbol{k}}}{w_{\boldsymbol{k}}} \cdot L^{\prime}\left(\xi(f), \Theta_{\Lambda}, 0\right),
$$

where $c_{f}^{+}(0,0)$ is the value of $c_{f}^{+}(0) \in \mathfrak{S}_{L}$ at the trivial coset in $L^{\vee} / L$.

Proof. First assume $f(\tau)=F_{m, \mu}(\tau)$ is the Hejhal-Poincaré series of $\S 3.2$, so that

$$
\widehat{\mathcal{Z}}(f)=\left(\mathcal{Z}(m, \mu), \Phi_{m, \mu}\right)=\widehat{\mathcal{Z}}^{\ominus}(m, \mu) \otimes \widehat{\boldsymbol{T}}^{\otimes R_{\Lambda}(m, \mu)} .
$$

By Proposition 5.6.2 there is a decomposition

$$
\begin{aligned}
{\left[\widehat{\mathcal{Z}}^{९}(m, \mu): \mathcal{Y}\right]=} & \sum_{\substack{m_{1}+m_{2}=m \\
m_{1}>0 \\
\left(\mu_{1}, \mu_{2}\right) \in(\mu+L) /\left(L_{0} \oplus \Lambda\right)}} R_{\Lambda}\left(m_{2}, \mu_{2}\right) \cdot \widehat{\operatorname{deg}} \mathcal{Z}_{0}\left(m_{1}, \mu_{1}\right) \\
& +\sum_{y \in \mathcal{Y}(\mathbb{C})} \frac{\Phi_{m, \mu}(y)}{\# \operatorname{Aut}(y)},
\end{aligned}
$$

where we view $\mathcal{Z}_{0}\left(m_{1}, \mu_{1}\right) \in \widehat{\operatorname{Div}}(\mathcal{Y})$ as an arithmetic divisor with trivial Green function. Theorem 


\section{F. Andreatta, E. Z. Goren, B. Howard, K. Madapusi Pera}

4.5.1 shows that the first term is

$$
\begin{gathered}
\sum_{\substack{m_{1}+m_{2}=m \\
m_{1}>0 \\
\left(\mu_{1}, \mu_{2}\right) \in(\mu+L) /\left(L_{0} \oplus \Lambda\right)}} R_{\Lambda}\left(m_{2}, \mu_{2}\right) \cdot \widehat{\operatorname{deg}} \mathcal{Z}_{0}\left(m_{1}, \mu_{1}\right) \\
=-\frac{h_{\boldsymbol{k}}}{w_{\boldsymbol{k}}} \sum_{\substack{m_{1}+m_{2}=m \\
m_{1}>0 \\
\left(\mu_{1}, \mu_{2}\right) \in(\mu+L) /\left(L_{0} \oplus \Lambda\right)}} a_{L_{0}}^{+}\left(m_{1}, \mu_{1}\right) R_{\Lambda}\left(m_{2}, \mu_{2}\right) \\
=-\frac{h_{\boldsymbol{k}}}{w_{\boldsymbol{k}}} \sum_{\substack{m_{1}+m_{2}=m \\
m_{1}>0}}\left\{c_{f}^{+}(-m), a_{L_{0}}^{+}\left(m_{1}\right) \otimes R_{\Lambda}\left(m_{2}\right)\right\} .
\end{gathered}
$$

For our special choice of $f(\tau)$ the constant term (5.21) simplifies to

$$
\begin{aligned}
\mathrm{CT}\left\{f^{+}, \mathcal{E}_{L_{0}} \otimes \Theta_{\Lambda}\right\}= & \left\{c_{f}^{+}(0), a_{L_{0}}^{+}(0) \otimes R_{\Lambda}(0)\right\} \\
& +\sum_{m_{1}+m_{2}=m}\left\{c_{f}^{+}(-m), a_{L_{0}}^{+}\left(m_{1}\right) \otimes R_{\Lambda}\left(m_{2}\right)\right\}
\end{aligned}
$$

and so Theorem 5.7.1 becomes

$$
\begin{aligned}
\sum_{y \in \mathcal{Y}(\mathbb{C})} \frac{\Phi_{m, \mu}(y)}{\# \operatorname{Aut}(y)}= & -\frac{h_{\boldsymbol{k}}}{w_{\boldsymbol{k}}} \cdot L^{\prime}\left(\xi(f), \Theta_{\Lambda}, 0\right)+\frac{h_{\boldsymbol{k}}}{w_{\boldsymbol{k}}}\left\{c_{f}^{+}(0), a_{L_{0}}^{+}(0) \otimes R_{\Lambda}(0)\right\} \\
& +\frac{h_{\boldsymbol{k}}}{w_{\boldsymbol{k}}} \sum_{m_{1}+m_{2}=m}\left\{c_{f}^{+}(-m), a_{L_{0}}^{+}\left(m_{1}\right) \otimes R_{\Lambda}\left(m_{2}\right)\right\} .
\end{aligned}
$$

We have now proved

$$
\begin{aligned}
{\left[\widehat{\mathcal{Z}}^{\bigcirc}(m, \mu): \mathcal{Y}\right]=} & -\frac{h_{\boldsymbol{k}}}{w_{\boldsymbol{k}}} \cdot L^{\prime}\left(\xi(f), \Theta_{\Lambda}, 0\right)+\frac{h_{\boldsymbol{k}}}{w_{\boldsymbol{k}}} \cdot\left\{c_{f}^{+}(0), a_{L_{0}}^{+}(0) \otimes R_{\Lambda}(0)\right\} \\
& +\frac{h_{\boldsymbol{k}}}{w_{\boldsymbol{k}}}\left\{c_{f}^{+}(-m), a_{L_{0}}^{+}(0) \otimes R_{\Lambda}(m)\right\} .
\end{aligned}
$$

Corollary 5.3.2 implies both

$$
c_{f}^{+}(0,0) \cdot[\widehat{\boldsymbol{T}}: \mathcal{Y}]=-\frac{h_{\boldsymbol{k}}}{w_{\boldsymbol{k}}} \cdot\left\{c_{f}^{+}(0), a_{L_{0}}^{+}(0) \otimes R_{\Lambda}(0)\right\},
$$

and

$$
R_{\Lambda}(m, \mu) \cdot[\widehat{\boldsymbol{T}}: \mathcal{Y}]=-\frac{h_{\boldsymbol{k}}}{w_{\boldsymbol{k}}} \cdot\left\{c_{f}^{+}(-m), a_{L_{0}}^{+}(0) \otimes R_{\Lambda}(m)\right\}
$$

leaving

$$
\left[\widehat{\mathcal{Z}}^{\ominus}(m, \mu): \mathcal{Y}\right]+\left[\widehat{\boldsymbol{T}}^{\otimes R_{\Lambda}(m, \mu)}: \mathcal{Y}\right]+c_{f}^{+}(0,0)[\widehat{\boldsymbol{T}}: \mathcal{Y}]=-\frac{h_{\boldsymbol{k}}}{w_{\boldsymbol{k}}} \cdot L^{\prime}\left(\xi(f), \Theta_{\Lambda}, 0\right),
$$

and proving the desired formula in the special case of $f=F_{m, \mu}$.

Now assume that $f$ satisfies $f^{+}(\tau)=O(1)$. In particular, $\mathcal{Z}(f)=\emptyset$ and the Green function $\Phi(f)$ is a smooth function on $\mathcal{M}(\mathbb{C})$. The arithmetic intersection is purely archimedean, and 


\section{Height PAIRINGS ON ORTHOgONAL ShIMURA VARIETIES}

Theorem 5.7.1 shows that

$$
\begin{aligned}
{[\widehat{\mathcal{Z}}(f): \mathcal{Y}] } & =\sum_{y \in \mathcal{Y}(\mathbb{C})} \frac{\Phi(f, y)}{\# \operatorname{Aut}(y)} \\
& =-\frac{h_{\boldsymbol{k}}}{w_{\boldsymbol{k}}} \cdot L^{\prime}\left(\xi(f), \Theta_{\Lambda}, 0\right)+\frac{h_{\boldsymbol{k}}}{w_{\boldsymbol{k}}} \cdot \operatorname{CT}\left\{f^{+}, \mathcal{E}_{L_{0}} \otimes \Theta_{\Lambda}\right\} \\
& =-\frac{h_{\boldsymbol{k}}}{w_{\boldsymbol{k}}} \cdot L^{\prime}\left(\xi(f), \Theta_{\Lambda}, 0\right)+\frac{h_{\boldsymbol{k}}}{w_{\boldsymbol{k}}} \cdot\left\{c_{f}^{+}(0), a_{L_{0}}^{+}(0) \otimes R_{\Lambda}(0)\right\} .
\end{aligned}
$$

Corollary 5.3.2 implies that

$$
\frac{h_{\boldsymbol{k}}}{w_{\boldsymbol{k}}} \cdot\left\{c_{f}^{+}(0), a_{L_{0}}^{+}(0) \otimes R_{\Lambda}(0)\right\}=\frac{h_{\boldsymbol{k}}}{w_{\boldsymbol{k}}} \cdot c_{f}^{+}(0,0) \cdot a_{L_{0}}^{+}(0,0)=-c_{f}^{+}(0,0) \cdot[\widehat{\boldsymbol{T}}: \mathcal{Y}],
$$

completing the proof in this case.

Finally, every weak harmonic Maass form can be written as a linear combination of the Hejhal-Poincaré series, and a form with holomorphic part $f^{+}(\tau)=O(1)$. Thus the claim follows from the linearity in $f$ of both sides of the desired equality.

\section{REFERENCES}

AGHM15 Fabrizio Andreatta, Eyal Z. Goren, Benjamin Howard, and Keerthi Madapusi Pera, Faltings heights of abelian varieties with complex multiplication, Preprint (2015).

Bas74 Hyman Bass, Clifford algebras and spinor norms over a commutative ring, Amer. J. Math. 96 (1974), no. 1, 156-206.

Bor98 Richard E. Borcherds, Automorphic forms with singularities on Grassmannians, Invent. Math. 132 (1998), no. 3, 491-562.

BF04 Jan Hendrik Bruinier and Jens Funke, On two geometric theta lifts, Duke Math. J. 125 (2004), no. 1, 45-90.

BHY15 Jan Hendrik Bruinier, Benjamin Howard, and Tonghai Yang, Heights of Kudla-Rapoport divisors and derivatives of L-functions, Invent. Math. (2015), in press.

BKY12 Jan Hendrik Bruinier, Steven S. Kudla, and Tonghai Yang, Special values of Green functions at big CM points, Int. Math. Res. Not. (2012), no. 9, 1917-1967.

Bru02 Jan Hendrik Bruinier, Borcherds products on $O(2, l)$ and Chern classes of Heegner divisors, Lecture Notes in Mathematics, Springer, 2002.

BY09 Jan Hendrik Bruinier and Tonghai Yang, Faltings heights of CM cycles and derivatives of L-functions, Invent. Math. 177 (2009), no. 3, 631-681.

Del72 Pierre Deligne, Travaux de Shimura, Séminaire Bourbaki vol. 1970/71 Exposés 382-399, Lecture Notes in Mathematics, Springer Berlin Heidelberg, 1972, pp. 123-165.

FC90 Gerd Faltings and Ching-Li Chai, Degeneration of abelian varieties, Ergebnisse der Mathematik und ihrer Grenzgebiete (3) [Results in Mathematics and Related Areas (3)], vol. 22, Springer-Verlag, Berlin, 1990.

FGA05 Barbara Fantechi, Lothar Göttsche, Luc Illusie, Steven L. Kleiman, Steven L., Nitin Nitsure, Angelo Vistoli, Fundamental algebraic geometry, Mathematical Surveys and Monographs, 123 (2005), American Mathematical Society, Providence, RI,

GS90 Henri Gillet and Christophe Soulé, Arithmetic intersection theory, Publications Mathématiques de l'Institut des Hautes Scientifiques 72 (1990), no. 1, 94-174.

Gro86 Benedict H. Gross, On canonical and quasi-canonical liftings, Invent. Math. 84 (1986), no. 2, 321-326. 
Gro03 Alexander Grothendieck, Revêtements étales et groupe fondamental (SGA 1), Documents Mathématiques (Paris), vol. 3, Société Mathématique de France, Paris, 2003.

Hida04 Haruzo Hida, p-adic automorphic forms on Shimura varieties, Springer Monographs in Mathematics, Springer Verlag, Berlin, Heidelberg, 2004.

How12 Benjamin Howard, Complex multiplication cycles and Kudla-Rapoport divisors, Ann. Math. 176 (2012), no. 2, 1097-1171.

HY12 Benjamin Howard and Tonghai Yang, Intersections of Hirzebruch-Zagier divisors and CM cycles, Lecture Notes in Mathematics, vol. 2041, Springer Verlag, Berlin, Heidelberg, 2012.

Hu99 Junda Hu, Specialization of Green forms and arithmetic intersection theory, Ph.D. thesis, University of Illinois Chicago, 1999.

KM15 Wansu Kim and Keerthi Madapusi Pera, 2-adic integral canonical models and the Tate conjecture in characteristic 2, Preprint. arXiv:1512.02540.

Kis10 Mark Kisin, Integral models for Shimura varieties of abelian type, J. Amer. Math. Soc. 23 (2010), no. 4, 967-1012.

Kud04 Stephen S. Kudla, Special cycles and derivatives of Eisenstein series, Heegner Points and Rankin L-Series, Math. Sci. Res. Inst. Publ., vol. 49, Cambridge University Press, Cambridge, 2004, pp. 243-270.

KR99 Stephen S. Kudla and Michael Rapoport, Arithmetic Hirzebruch-Zagier cycles, J. Reine Angew. Math. 515 (1999), 155-244.

KR00 Cycles on Siegel threefolds and derivatives of Eisenstein series, Ann. Sci. École Norm. Sup. (4) 33 (2000), no. 5, 695-756.

KRY99 Stephen S. Kudla, Michael Rapoport, and Tonghai Yang, On the derivative of an Eisenstein series of weight one, Internat. Math. Res. Notices 7 (1999), 347-385.

KRY04 Derivatives of Eisenstein series and Faltings heights, Compos. Math. 140 (2004), no. $04,887-951$.

KRY06 Modular forms and special cycles on Shimura curves. (AM-161), Annals of Mathematics Studies, Princeton University Press, 2006.

KY13 Stephen S. Kudla and Tonghai Yang, On the pullback of an arithmetic theta function, Manuscripta Math. 140 (2013), no. 3-4, 393-440.

MP16 Keerthi Madapusi Pera, Integral canonical models for spin Shimura varieties, Compos. Math. 152 (2016), no. 4, 769- 824.

MFK94 David Mumford, John Fogarty, and Frances Kirwan, Geometric Invariant Theory, Ergebnisse der Mathematik und ihrer Grenzgebiete (2) [Results in Mathematics and Related Areas (2)], vol. 34, Springer-Verlag, Berlin, 1994.

Sch09 Jarad Schofer, Borcherds forms and generalizations of singular moduli, J. Reine Angew. Math. 629 (2009), 1-36.

Tsi15 Jacob Tsimerman, A proof of the André-Oort conjecture for $A_{g}$, Preprint (2015).

vdG88 Gerard van der Geer, Hilbert modular surfaces, Ergebnisse der Mathematik und ihrer Grenzgebiete (3) [Results in Mathematics and Related Areas (3)], vol. 16, Springer-Verlag, Berlin, 1988.

Vis89 Angelo Vistoli, Intersection theory on algebraic stacks and on their moduli spaces, Invent. Math. 97 (1989), no. 3, 613-670.

Yan10 Tonghai Yang, The Chowla-Selberg formula and the Colmez conjecture, Can. J. Math. 62 (2010), no. 2, 456-472. 
Height PAIRINGs on ORThOgOnal Shimura varieties

Fabrizio Andreatta fabrizio.andreatta@unimi.it

Dipartimento di Matematica "Federigo Enriques", Università di Milano, via C. Saldini 50, Milano, Italia

Eyal Z. Goren eyal.goren@mcgill.ca

Department of Mathematics and Statistics, McGill University, 805 Sherbrooke St. West, Montreal, QC, Canada

Benjamin Howard howardbe@bc.edu

Department of Mathematics, Boston College, 140 Commonwealth Ave, Chestnut Hill, MA, USA

Keerthi Madapusi Pera keerthi@math.uchicago.edu

Department of Mathematics, University of Chicago, 5734 S University Ave, Chicago, IL, USA 